\title{
2015 ANNUAL REPORT ARGONNE LEADERSHIP COMPUTING FACILITY
}


On the cover A snapshot of an

expanding detonation wave from a

visualization that depicts weak ignition

behind a reflected shock in a $2 \mathrm{H}_{2}+\mathrm{O}_{2}$

mixture at initially atmospheric pressure..

Image credit: Charles Bacon, Marta

García, and Joseph A. Insley, Argonne

National Laboratory; Alexei Khokhlov, The

University of Chicago; Joanna Austin and

Andrew Knisely, University of Illinois at

Urbana-Champaign 


\section{ARGONNE LEADERSHIP COMPUTING FACILITY ANNUAL REPORT 2015}




\title{
TABLE OF CONTENTS
}

\author{
4 About ALCF \\ 5 Director's Message \\ 6 ALCF Leadership: Year in Review \\ 8 Introducing Aurora and Theta \\ 10 Preparing Theta for Science on Day One \\ 12 ALCF Resources Propel Data-Intensive Experiments \\ 14 Allocation Programs \\ 162015 Mira Usage \\ 18 SCIENCE \\ 20 Computational Design of Interfaces \\ for Photovoltaics • Noa Marom \\ 21 First-Principles Simulations of High-Speed \\ Combustion and Detonation - Alexei Khokhlov \\ 22 Towards Breakthroughs in Protein Structure \\ Calculation and Design - David Baker
}

23 Performance Improvement of CFD Code Converge on Blue Gene/Q Systems - Sibendu Som and Marta García

24 Atomistic Computational Model of Radiation Damage of Nano-Sized Systems in Intense X-Ray Pulses • Linda Young

25 Computations for the Development of the Nanoporous Materials Genome • J. Ilja Siepmann

26 Dynamics of Conformational Transition in Polymer Grafted Nanoparticles • Subramanian Sankaranarayanan

27 Reactive MD Simulations of Electrochemical Oxide Interfaces at Mesoscale - Subramanian Sankaranarayanan 
$28 \mathrm{SiO}_{2}$ Fracture: Chemomechanics with a MachineLearning Hybrid QM/MM Scheme - James Kermode

29 High-Fidelity Simulation of Tokamak Edge Plasma Transport • C.S. Chang

30 Simulation of Large Hadron Collider Events Using Leadership Computing - Thomas LeCompte

\section{PEOPLE}

35 ALCF Expertise

36 ALCF Welcomes Two New Directors

38 Staff Spotlights

\section{$40 \mathrm{TECH}$}

42 Enabling Science Through HPC Technology

45 New Systems Provide Advanced Capabilities

46 ALCF Computing Resources

\section{OUTREACH}

50 Shaping the Future of Supercomputing

52 Growing the HPC Community

\section{APPENDICES}

57 Publications

65 Projects 


\section{ABOUT ALCF}

\section{The Argonne Leadership Computing Facility provides supercomputing capabilities to the scientific and engineering community to advance fundamental discovery and understanding in a broad range of disciplines.}

Supported by the U.S. Department of Energy's (DOE's) Office of Science, Advanced Scientific Computing Research (ASCR) program, the ALCF is one of two DOE Leadership Computing Facilities in the nation dedicated to open science. Available to researchers from universities, industry, and government agencies, the ALCF is a DOE user facility that helps accelerate the pace of discovery and innovation by providing supercomputing resources that are 10 to 100 times more powerful than systems typically used for scientific research.
Through substantial awards of supercomputing time and user support services, the ALCF enables large-scale modeling and simulation research aimed at solving some of the world's largest and most complex problems in science and engineering. 


\section{DIRECTOR'S MESSAGE}

2015 marked 10 years since Argonne installed its first IBM Blue Gene system-a 5-teraflops machine that was both a prototype and a proving ground for what would become the Argonne Leadership Computing Facility in 2006. The BG/L was the latest in a long line of parallel computing systems that Argonne mathematicians and computer scientists procured and experimented with starting in the early 1980 s.

That same machine supported the first six Department of Energy (DOE) Innovative and Novel Computational Impact on Theory and Experiment (INCITE) projects at Argonne, and solidified a decade-long industry partnership that has produced some of the world's most powerful machines supporting high-end computational science and engineering.

Today, ALCF's main resource, Mira, an IBM Blue Gene/Q, is 2,000 times more powerful than Argonne's BG/L. It is the last in a very successful series of computers on which the research community has solved some of the most pressing problems facing our planet. And now we look to the future.

Of course, it was big news in 2015 when DOE announced Argonne's next-generation systems. The 180-petaflops supercomputer, known as Aurora, is planned to arrive in 2018, and will be a massively many-core system based on Intel's Knights Hill Xeon Phi processor technology and integrated on Cray's next-generation hardware platform. Preceding Aurora's arrival is Theta, based on Intel's Knights Landing technology, slated for installation in late 2016.

In 2015, the ALCF supported 34 INCITE projects, 25 ASCR Leadership Computing Challenge projects, and more than 190 Director's Discretionary projects. The facility also provided short-term strategic computational runs to science teams working in other major facilities to run analysis jobs on their data-intensive experiments. These included high-energy $x$-ray beamline teams, a partnership pursuing breakthroughs in battery research, and physicists using the world's most powerful particle acceleratorall of which are described in the following pages.

On the technical side, ALCF researchers made progress on several efforts, such as GLEAN and AutoPerf, aimed at improving high-performance computing (HPC) in leadership-class facilities and beyond. The operations team launched Cooley, a powerful new visualization and analysis cluster with terabytes of RAM and GPU memory

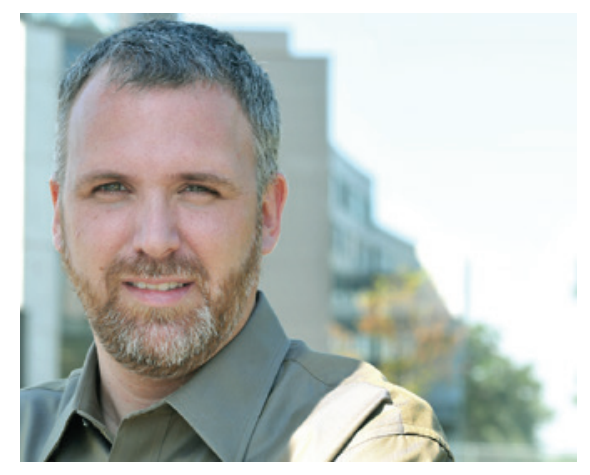

\section{Michael E. Papka}

Division Director, ALCF; Deputy Associate Laboratory Director, Computing, Environment, and Life Sciences

to help meet our user community's data analysis needs. Cooley, the follow-on system to Tukey, will support more exploration capabilities, including in-situ analysis and unprecedented volume-rendered visualization.

On the outreach side, ALCF staff promoted our services and world-class technical expertise within the HPC community, and continued to build on our successful training programs for our current and future users alike. The ALCF has hit its stride in many areas, and will no doubt continue to impact the greater HPC community through its research publications, software releases, and active involvement in standards bodies and working groups.

Our staff remains the ALCF's most valuable asset, and in this year's report we showcase a diversity of them: computational scientists Marta García and Chris Knight, application performance engineer Scott Parker, operations team lead Skip Reddy, postdoc and visualization expert Silvio Rizzi, and UX developer Savannah French. 2015 also saw major leadership changes, with Katherine Riley replacing Paul Messina as our second Director of Science, and Mark Fahey replacing Bill Allcock as our second Director of Operations.

This is my final annual letter to you as ALCF's director. After more than five years as director, I am returning to my full-time role as Argonne's Deputy Associate Laboratory Director for Computing, Environment, and Life Sciences, where I will continue to cultivate and support efforts that connect computing to areas of inquiry. I'm proud of the time I've spent at the ALCF and all the things the division has accomplished. I look forward to sharing in its future successes.

\section{Michael E. Papka}




\section{ALCF LEADERSHIIP: YEAR IN REVIEW}

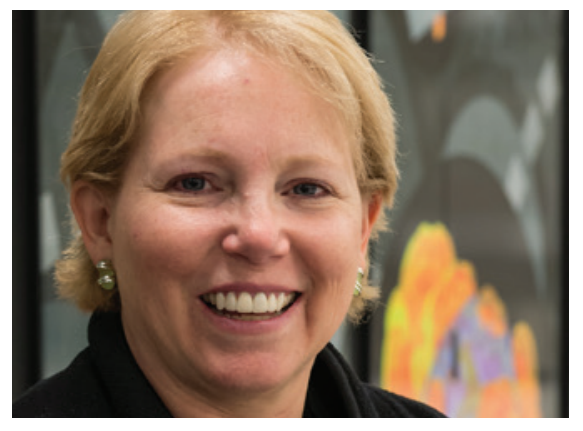

\section{FUTURE SYSTEMS AND FACILITY INITIATIVES}

\section{Susan Coghlan \\ Deputy Director}

In April, DOE's announcement of our two future systems, Theta and Aurora, made national news. The contracts to design and build the supercomputers were awarded to Intel and Cray. Like Mira, both machines will be many-core, homogeneous systems, which will help users with the transition from our current IBM architecture to the Intel Xeon Phi architecture. We continued to work diligently behind the scenes to prepare for the arrival of the new systems, making progress with facility enhancements to address their power and cooling requirements. In addition, we kicked off our Early Science Program for Theta and we began working closely with Intel and Cray to evaluate early hardware and to improve the system software.
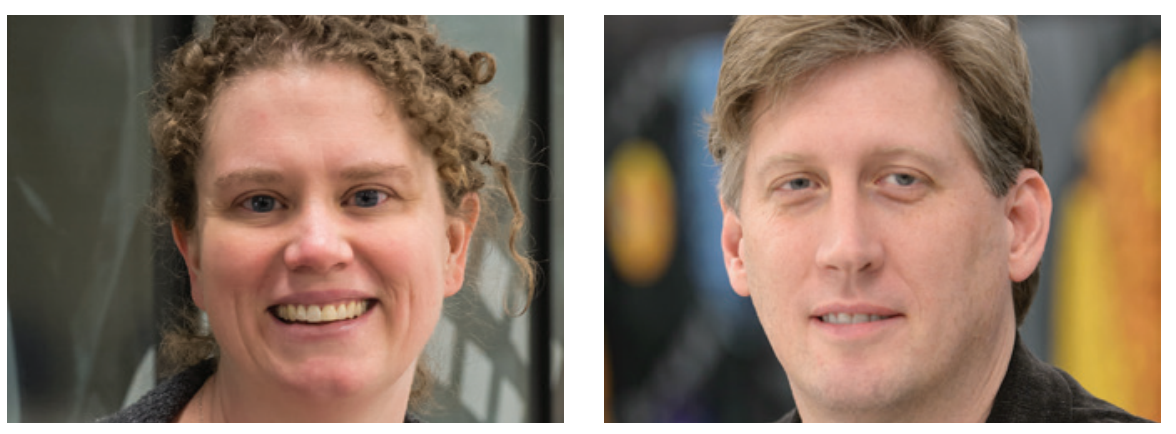

\section{SCIENCE}

\section{Katherine Riley}

Director of Science

In October, I was named the ALCF's new Director of Science, the second in the facility's history. I'm excited about my role in shaping the ALCF's future as a world-class scientific computing center. We started phase one of our Early Science Program this year to help ready our new machines for science. The selected projects will also lead the way in demonstrating how applications can prepare for many of the architectural features we will see as we move toward exascale. In September, I chaired DOE's HighPerformance Computing Operations Review (HPCOR) to identify ways to improve application portability on future systems. We also collaborated with OLCF and NERSC on the first two Exascale Requirements Reviews, which aim to determine the future computational science objectives for the six DOE Office of Science programs. Finally, Mira continued to enable our users to produce amazing science and engineering research, resulting in many high-impact publications.

\section{OPERATIONS}

\section{Mark Fahey}

Director of Operations

In my first year as ALCF Director of Operations, I'm pleased to report that Mira had its best performance year ever in 2015 - hitting or exceeding all key metrics for availability and utilization. We also installed a new visualization and analysis cluster with 386 GB of memory and a GPU per node, called Cooley, and decommissioned its predecessor. To improve our archival storage, we completed the swap of roughly 10,000 LTO4 tapes to LTO6 tapes, providing nearly three times the capacity. In collaboration with Argonne's Joint Laboratory for System Evaluation (JSLE), we piloted a data management service called Petrel that allows researchers to store and share large datasets internally and externally. This effort involved performing upgrades to the JLSE network, which resulted in 40/56 Gb Ethernet connectivity. In addition, we deployed a suite of tools to track compiler and library usage on our Blue Gene/Q systems. In the coming year, we will be deploying a new Cray XC system, a new "burst buffer-like" storage cache, a hierarchical storage management disk-to-tape migration solution, and a new allocation charging system. 

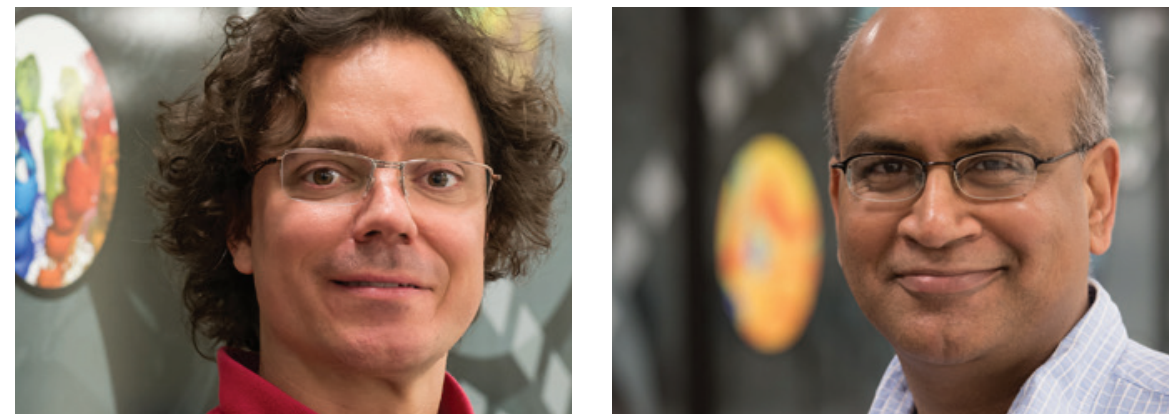

\section{USER \\ EXPERIENCE}

\section{Richard Coffey}

Director of User Experience

We have many evolving practices in place to help ensure a smooth and successful user experience at the ALCF. From day one, the user experience team interacts with the project teams nearly continuously; from helping them quickly gain access to the resources to providing training to promoting their accomplishments. In 2015, we exceeded all targets related to user support and problem resolution. We had our best-ever response rate for our annual user survey. We designed and executed our first-ever "Ensemble Jobs for Better Throughput" videoconference. And, I'm especially proud to report, every one of our 2016 INCITE projects were ready for prime time two weeks prior to the critical January 1 start date. All of the efforts helped remove roadblocks and enable scientists to focus on their research. I look forward to the challenges ahead. Great service is our goal and we never tire of finding solutions to problems.

\section{PERFORMANCE ENGINEERING}

\section{Kalyan Kumaran}

Manager, Performance Engineering

We had a very busy year, supporting users on Mira while also playing a critical technical role in preparations for our next-generation systems, Theta and Aurora. One of the highlights involving our current systems was the deployment of AutoPerf, a library that automatically collects performance data from applications. This tool is already proving to be tremendously helpful in our efforts to optimize applications and understand their requirements. For our future systems, we helped complete the non-recurring engineering (NRE) and build contracts and led NRE working groups to ensure that the new technologies meet the needs of the facility and our users. Our team also began porting applications and benchmarks to early hardware to reveal information on the Intel Xeon Phi architecture that will help ease the transition from Mira to Theta. In addition, we are leading a Theta Early Science Program project to port performance tools, debuggers, libraries, and other software to Theta to make them available when the system enters production mode next year.

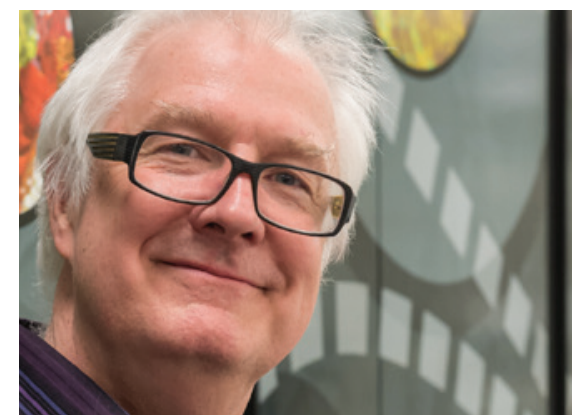

\section{VISUALIZATION AND DATA ANALYSIS}

\section{Mark Hereld}

Manager, Visualization and Data Analysis

In June, we launched our new visualization and analysis platform, Cooley. With eight times the memory capacity of its predecessor, Tukey, Cooley improved our ability to visualize, delve into, and interact with large-scale numerical datasets. For instance, the new system enabled an INCITE team to perform massive 3D visualizations of high-speed combustion and detonation that were intractable on Tukey. In addition to helping scientists better understand and present their simulation results, Cooley also generated some visualizations that garnered noteworthy attention. We worked with an INCITE research team to produce a visualization of superlubricity that went on to be featured on the cover of DOE Quadrennial Technology Review. We also worked with Argonne engine researchers to generate a gasoline compression ignition visualization that was recognized as a finalist in the SC15 Visualization Showcase. We are looking forward to another year of helping our users to achieve research goals by turning their simulation data into illuminating visualizations. 


\section{INTRODUCING}
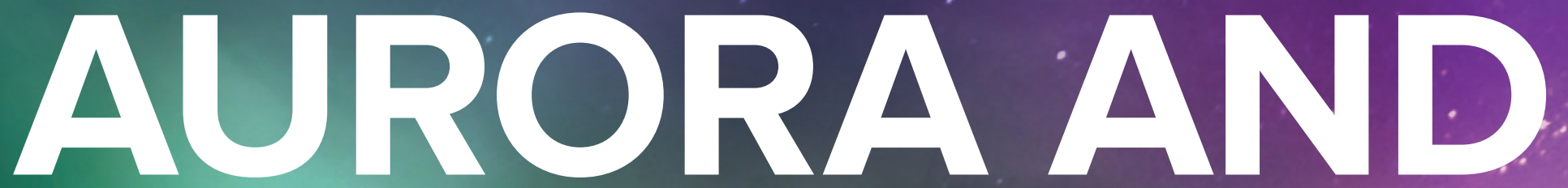

\section{In April, DOE announced its investment to deliver Aurora and Theta, the ALCF's next-generation supercomputers.}
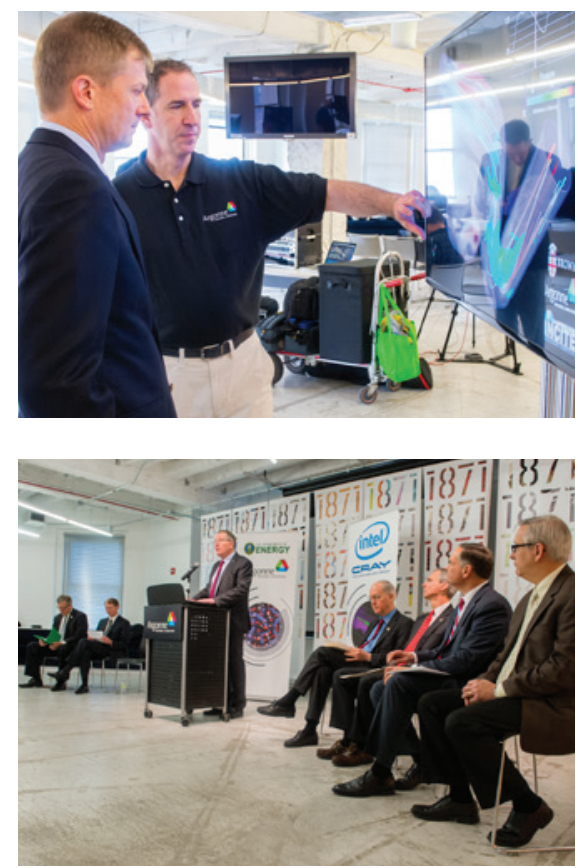

Top: ALCF visualization expert Joe Insley explains a blood flow simulation to a guest following the April 9 press event announcing Aurora and Theta.

Bottom: Argonne Director Peter Littlewood addresses the press and invited guests during the DOE announcement.
Designed in collaboration with industry leaders Intel and Cray, Aurora is scheduled for delivery in 2018. The effort is the result of DOE's Collaboration of Oak Ridge, Argonne, and Lawrence Livermore (CORAL) initiative, a joint procurement activity launched in 2014.

\section{Aurora will deliver more than} 18 times the computational performance of Mira, its predecessor at the ALCF, using a nearly equal number of compute nodes. Aurora will be a manycore system, but with nearly an order of magnitude more processors.
Aurora's revolutionary architecture features Intel's HPC scalable system framework and second-generation Intel ${ }^{\circledR}$ OmniPath Fabric, providing a peak performance of 180 petaflops. The system will have a combined total of over 7 petabytes of on-package high-bandwidth memory and persistent memory,

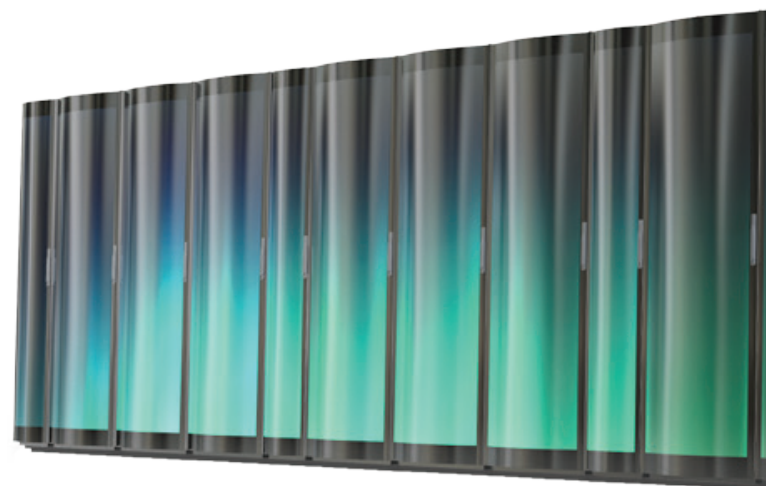




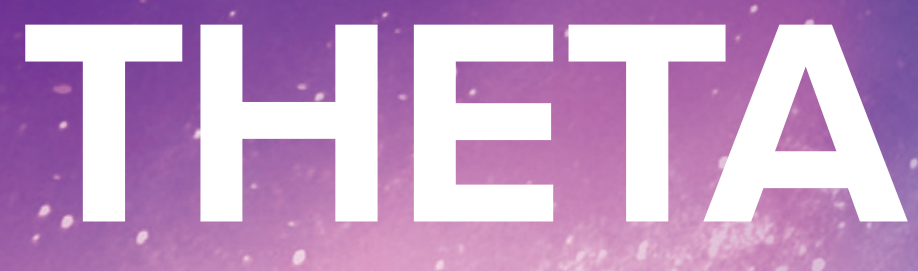

connected and communicating via a high-performance system fabric to achieve landmark throughput. The nodes will be linked to a dedicated burst buffer and a high-performance parallel storage solution.

The system will help ensure continued U.S. leadership in high-end computing for scientific research, while also cementing the nation's position as a global leader in the development of next-generation exascale computing systems.
A second system, named Theta, will be delivered in 2016 to serve as a bridge between Mira and Aurora. Based on Intel's secondgeneration Xeon Phi processor, Theta will enable breakthrough science and engineering research while providing an early production system to help ALCF users transition their applications to the new technology.

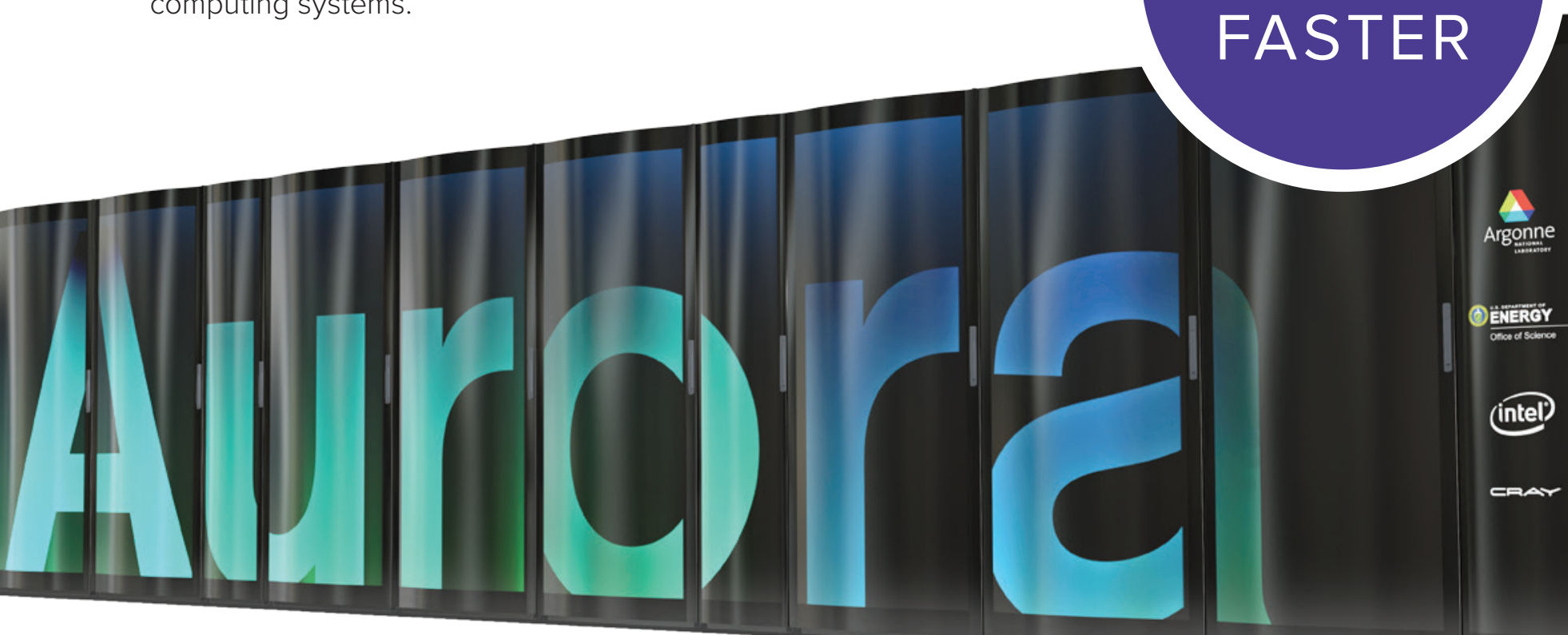




\title{
PREPARING THETA FOR SCIENCE ON DAY ONE
}

\author{
As part of the process of bringing a new supercomputer into \\ production, the ALCF conducts the Early Science Program (ESP) to \\ ensure its next-generation systems are ready to hit the ground running.
}

In August, the facility kicked off its Theta ESP with the selection of six computational science projects to help prepare scientific applications for the architecture and scale of the new supercomputer.

The ESP brings together computational scientists, code developers, and computing hardware experts to optimize key applications for Theta, and to solidify libraries and infrastructure to pave the way for other applications to run on the system.

Modeled after the ALCF's highly successful ESP for Mira, the program also gives researchers substantial allocations of pre-production compute time on Theta to pursue innovative computational science calculations that push the boundaries of what's possible with leadership-class supercomputers.
Like the typical ALCF workload, the six selected ESP projects, known as Tier One projects, represent a wide spectrum of scientific areas and numerical methods.To help develop and optimize their software for Theta, project teams are collaborating with ALCF and vendor staff. Four of the six projects were also assigned a dedicated postdoctoral researcher.

Because of the strong response to the call for proposals, the ALCF expanded the Theta ESP to include six additional Tier Two projects to help prepare other applications for Theta. While these projects did not receive allocations for science runs, they do have access to ESP training, an ESP discussion forum, early hardware, and to Theta itself for porting, tuning, and debugging. In addition, the ALCF is leading an omnibus ESP project to port and adapt software tools, such as programming models, performance tools, debuggers, and libraries, so they are available during the system's early deployment phase.

Once Theta is delivered and accepted, the Early Science period will give ESP teams dedicated access to the full system before Theta enters production mode. The Theta ESP will culminate with a community workshop, open to all ALCF users, in which the project teams present their code development work and share lessons learned.

\section{From Mira to Aurora}

\begin{tabular}{|c|c|c|c|c|c|c|c|c|c|c|c|c|c|c|c|c|c|}
\hline \multirow[b]{2}{*}{ Milestones* } & \multicolumn{3}{|l|}{ CY2015 } & \multicolumn{4}{|c|}{ CY2016 } & \multicolumn{4}{|c|}{ CY2017 } & \multicolumn{4}{|c|}{ CY2018 } & \multicolumn{2}{|c|}{ CY2019 } \\
\hline & Q1 Q2 & Q3 & Q4 & Q1 & Q2 & Q3 & Q4 & & Q2 & Q3 & Q4 & Q1 & Q2 & Q3 & Q4 & Q1 & Q2 \\
\hline \multicolumn{18}{|l|}{ Mira Production } \\
\hline \multicolumn{18}{|c|}{ Theta ESP Call for Proposals } \\
\hline \multicolumn{18}{|c|}{ Theta ESP Projects Announced } \\
\hline \multicolumn{18}{|c|}{ Theta ESP Application Preparation } \\
\hline \multicolumn{18}{|c|}{ Theta Early Science Period } \\
\hline \multicolumn{18}{|l|}{ Theta Production } \\
\hline \multicolumn{18}{|c|}{ Aurora ESP Call for Proposals } \\
\hline \multicolumn{18}{|c|}{ Aurora ESP Projects Announced } \\
\hline \multicolumn{18}{|c|}{ Aurora ESP Application Preparation } \\
\hline \multicolumn{18}{|c|}{ Aurora Early Science Period } \\
\hline Aurora Production & & & & & & & & & & & & & & & & & \\
\hline
\end{tabular}

\footnotetext{
* Dates are subject to change.
} 


\section{Theta ESP Tier One Science Projects}

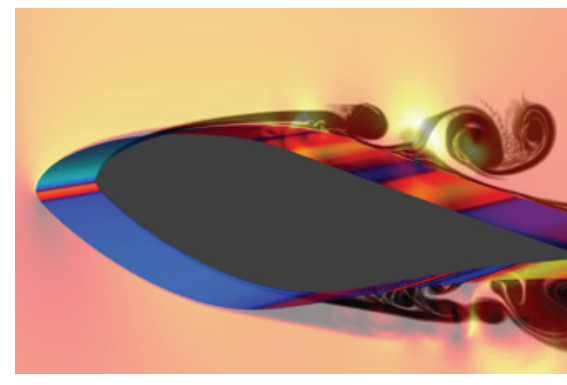

Scale-Resolving Simulations of Wind Turbines with SU2

\section{Juan J. Alonso}

Stanford University

\section{Code: SU2}

Researchers will develop a simulation capability to design better wind turbines and to lay out large wind farms for maximum energy extraction and improved turbine fatigue life. To do so, the research team will generate a database of large-eddy simulations of various single and multiple wind turbine settings.

Image credit: Ramesh Balakrishnan, Argonne National Laboratory

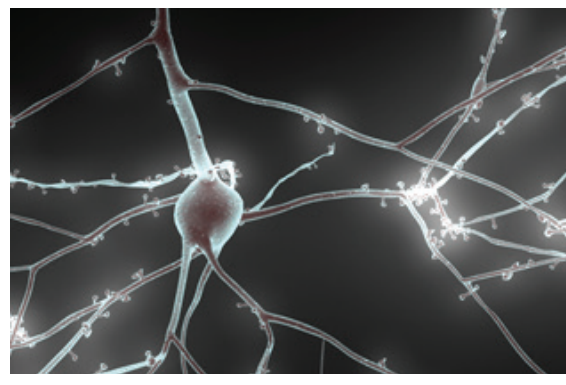

Large-Scale Simulation of Brain Tissue: Blue Brain Project, EPFL Fabien Delalondre EPFL

Code: CoreNeuron

This project aims to improve our understanding of the brain using simulations of brain plasticity-experience-dependent changes in synaptic connectivity. Other work will include simulating rodent somatosensory cortex and the electrical activity of the largest possible brain model for several seconds of biological time. Image credit: Blue Brain Project, EPFL

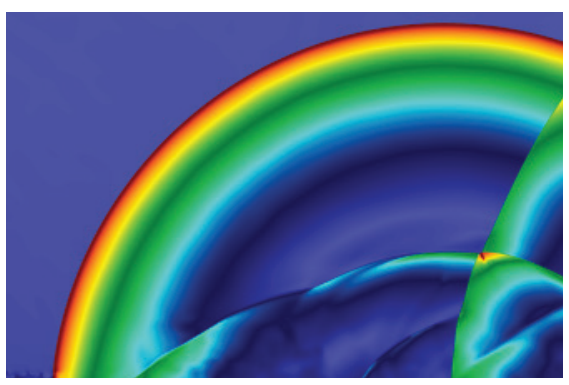

Direct Numerical Simulations of Flame Propagation in HydrogenOxygen Mixtures in Closed Vessels Alexei Khokhlov

The University of Chicago Code: $H S C D$

Researchers will perform direct numerical simulations of the flame acceleration and the deflagration-to-detonation transition process in hydrogen-oxygen mixtures in closed spherical vessels-exactly matching experimental apparatus. This research is aimed at improving the industrial and public safety of hydrogen fuels and certain water-cooled nuclear reactors.

Image credit: Charles Bacon, Marta García, and Joseph A. Insley, Argonne National Laboratory; Alexei Khokhlov, The University of Chicago; Joanna Austin and Andrew Knisely, University of Illinois at Urbana-Champaign

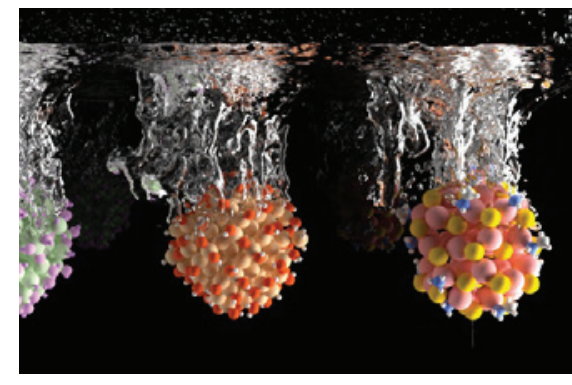

First-Principles Simulations of Functional Materials for Energy Conversion

Giulia Galli

The University of Chicago

Codes: Qbox, WEST

Researchers will combine ab initio molecular dynamics and post-density functional theory methods to optimize properties of nanostructured materials for use in solar and thermal energy conversion devices at an unprecedented level of accuracy. The ultimate goal is to provide a truly predictive tool for device performance within a Materials Genome Initiative design framework. Image credit: Nicholas Brawand, The University of Chicago

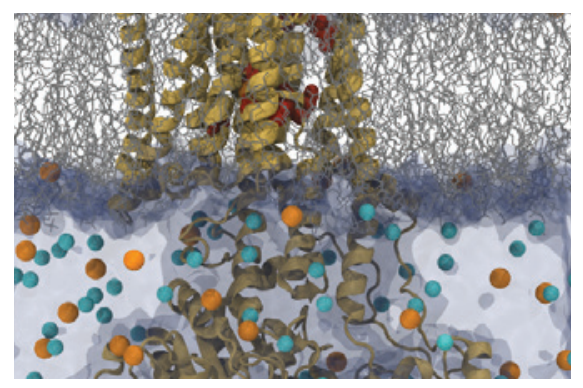

Free Energy Landscapes of Membrane Transport Proteins Benoît Roux

The University of Chicago Code: NAMD

This project will carry out molecular dynamics simulations to provide detailed visualizations of the large conformational changes of membrane transport proteins and quantitative predictions of the energetics of these processes. This atomistic picture of membrane transport proteins stands to improve our understanding of a broad range of biological functions.

Image credit: Brian Radak and Huan Rui, The University of Chicago 


\section{ALCF}

RESOURCES

PROPEL

DATA-INTENSIVE EXPERIMENTS

\section{Simulations have become an}

\section{essential part of experimental} research, providing information to help scientists test theories, understand results from experiments, and inform the setup of new experiments.

In 2015, the ALCF continued to build partnerships with experimental facilities that have large computation and data science challenges. The following collaborative efforts demonstrate how leadership computing resources can be used to propel scientific breakthroughs, and inform and facilitate large-scale, data-driven experiments.

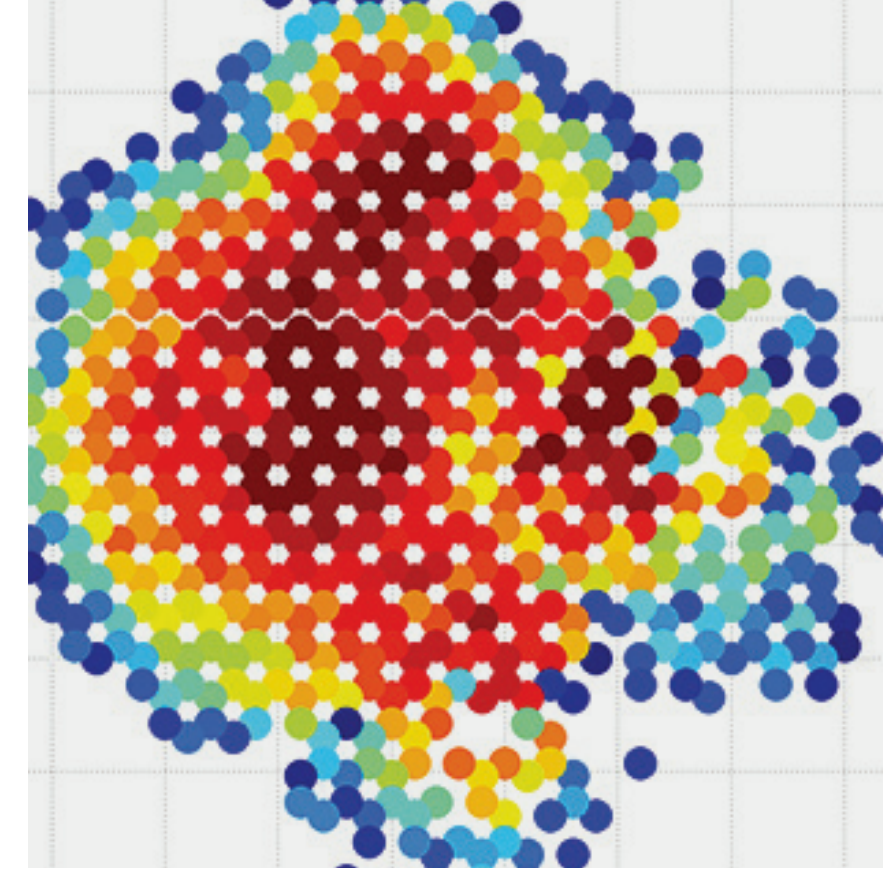

High-energy x-ray diffraction microscopy image of an $~ 50$ micron diameter gold wire (courtesy of B. Suter, Carnegie Mellon University) showing confidence index for each $\sim 1$ micron voxel. The data is from the 1-ID beamline of the APS. Reconstructions conducted using APS and ALCF resources were led by Argonne researchers Hemant Sharma and Justin Wozniak.

Image credit: Jonathan Almer and Hemant Sharma, Argonne National Laboratory

\section{Advanced Photon Source}

Recent work by a collaboration of scientists from Argonne's Advanced Photon Source (APS), Mathematics and Computer Science (MCS) Division, and the ALCF has produced advances in computational methods and infrastructure that are helping to boost APS beamline performance.

As part of a high-energy diffraction microscopy (HEDM) project, a research team received a three-day allocation of APS beam time to develop new experimental techniques and algorithms to image the grain structures of polycrystalline materials. They also received a Director's Discretionary allocation at the ALCF to run an analysis job of their experimental procedure on Mira. Using the Swift parallel scripting language, MCS and ALCF researchers helped transform their HEDM analysis software into a scalable application capable of utilizing Mira efficiently.

The resulting simulations showed obviously skewed data, which allowed the APS researchers to adjust the experimental hardware and take new trial images until the confidence levels indicated a correct physical setup. The culprit was a cable that was too short, constraining the detector's movement. Without such inbeam experimentation and computational assessment, the researchers' beam time would have proceeded to completion, yielding useless data that would be discovered weeks or months later during a manual data analysis phase. By using Mira to help detect and correct a problem early on, the three-day experiment instead produced publishable results. 


\section{ALLOCATION PROGRAMS}

\section{Any researcher in the world with a large-scale computing problem can apply for time on ALCF computing resources.}

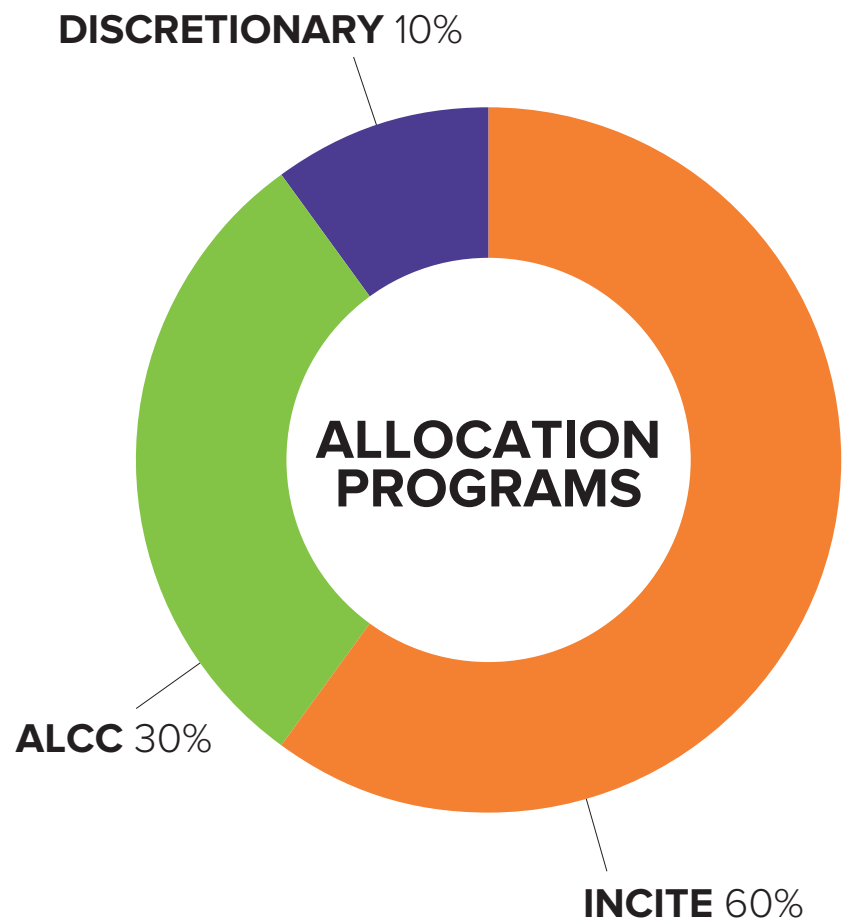

A breakdown of how computing time on Mira is allotted among the three primary allocation programs.

\section{Innovative \& Novel \\ Computational Impact on Theory and Experiment (INCITE)}

The DOE's INCITE program provides allocations to computationally intensive, large-scale research projects that aim to address "grand challenges" in science and engineering. The program conducts a two-part review of all proposals: a peer review by an international panel of experts and a computational-readiness review. The annual call for proposals is issued in April, and the allocations are awarded in millions of core-hours for one to three years.

\section{ASCR Leadership Computing Challenge (ALCC)}

The DOE's ALCC program allocates resources to projects directly related to the DOE's energy mission, national emergencies, or for broadening the community of researchers capable of using leadership computing resources. The DOE conducts a peer review of all proposals based on scientific and technical merit of the project; appropriateness of the proposed method or approach; competency and adequacy of personnel and proposed resources; and the reasonableness and appropriateness of the proposed allocation request. The yearlong allocation cycle runs from July 1 to June 30.

\section{Director's Discretionary}

The ALCF's Director's Discretionary program provides "start up" awards to researchers working toward an INCITE or ALCC allocation to help them achieve computational readiness. Projects must demonstrate a need for leadership-class resources. Awards may be made year round to industry, academia, laboratories, and others, and are usually between three and six months in duration. The size of the award varies based on the application and its readiness/ability to scale; awards are generally from the low hundreds of thousands to the low millions of core-hours. 

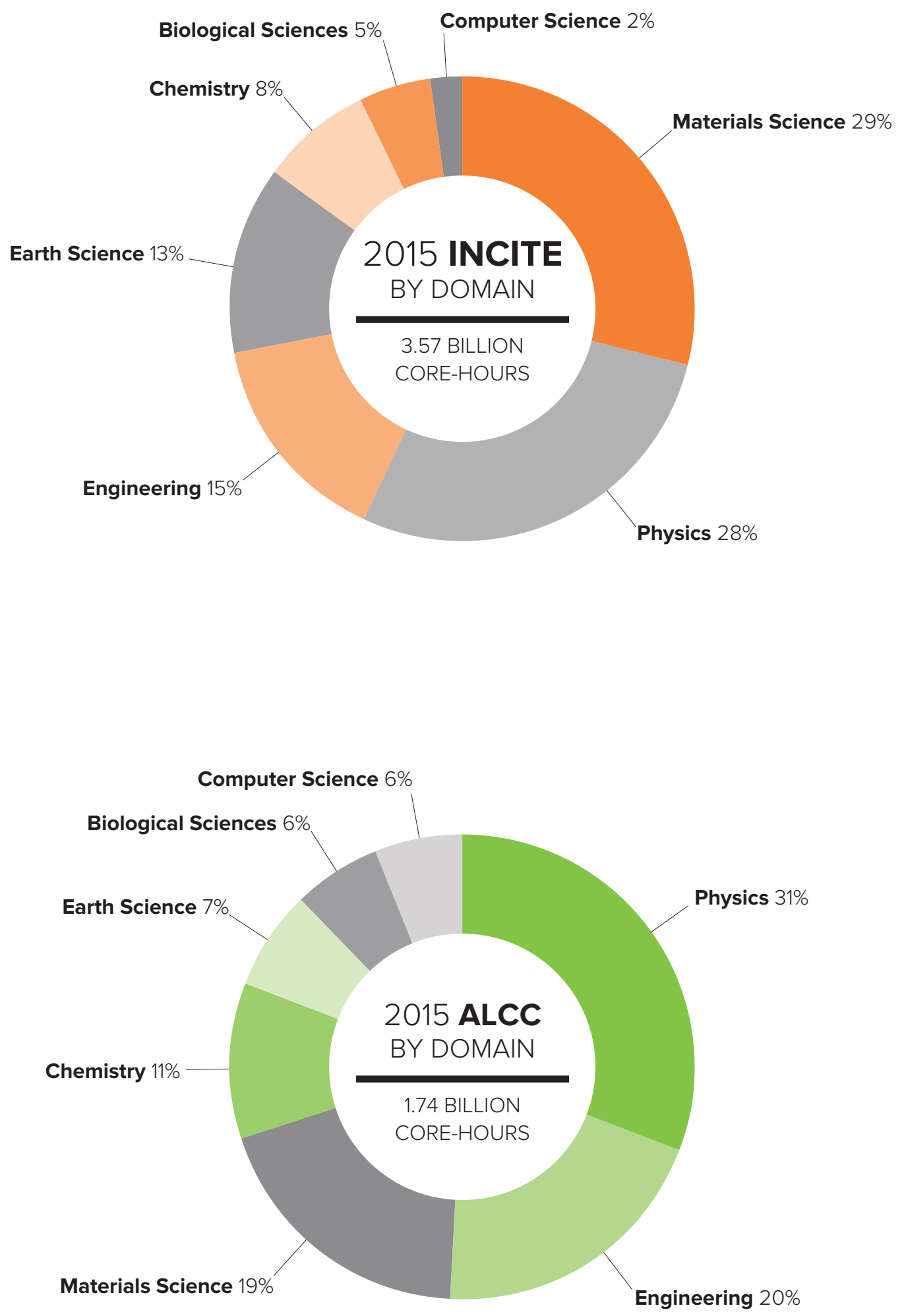

Note: Data is from calendar year 2015. 


\section{MIRA USAGE}

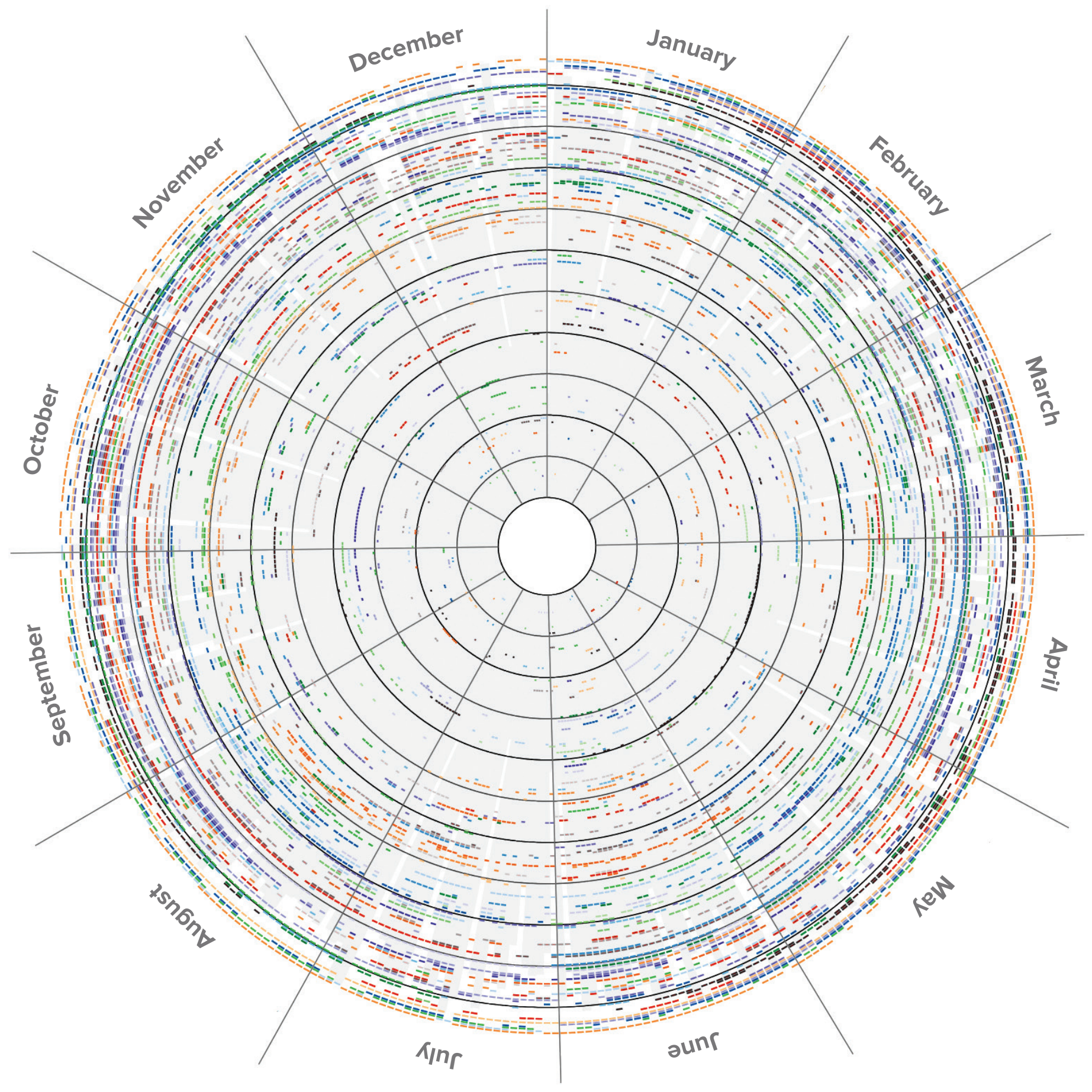

This visualization depicts all projects and jobs that ran on Mira in 2015 Each colored ring represents a project; each box represents a day that the project ran a job on the system. The radial histogram in the background shows the percentage of overall machine usage for that day (with 0 percent at the inner circle and 100 percent at the outer edge of the circle).

Image credit: Michael E. Papka and Eric Pershey, Argonne National Laboratory; Adam Young, Northern Illinois University 


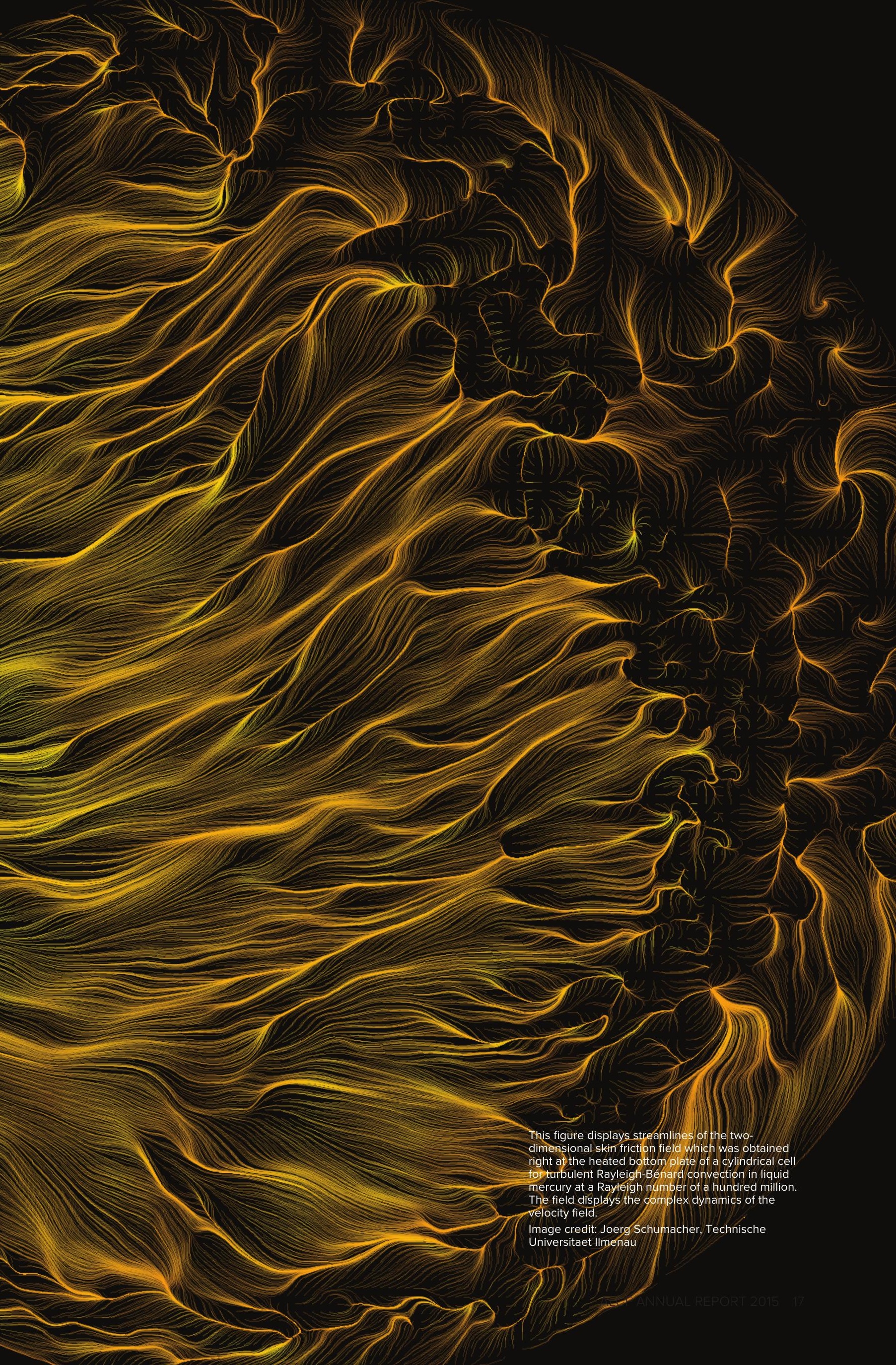


The ALCF is accelerating scientific discoveries in many disciplines, ranging from chemistry and engineering to physics and materials science.

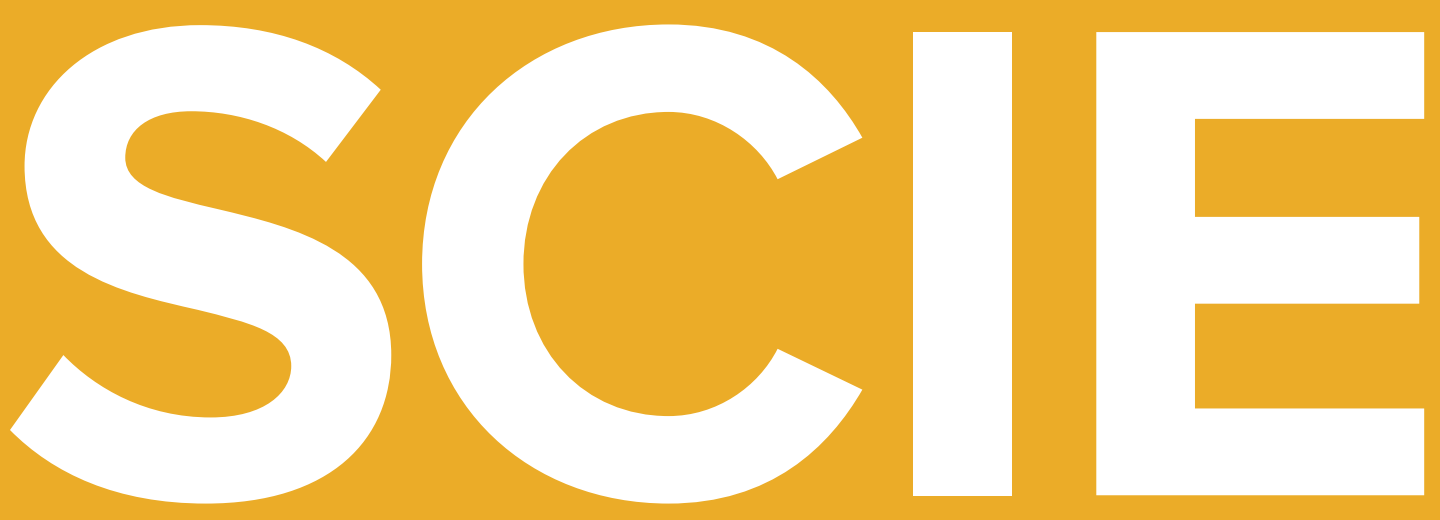




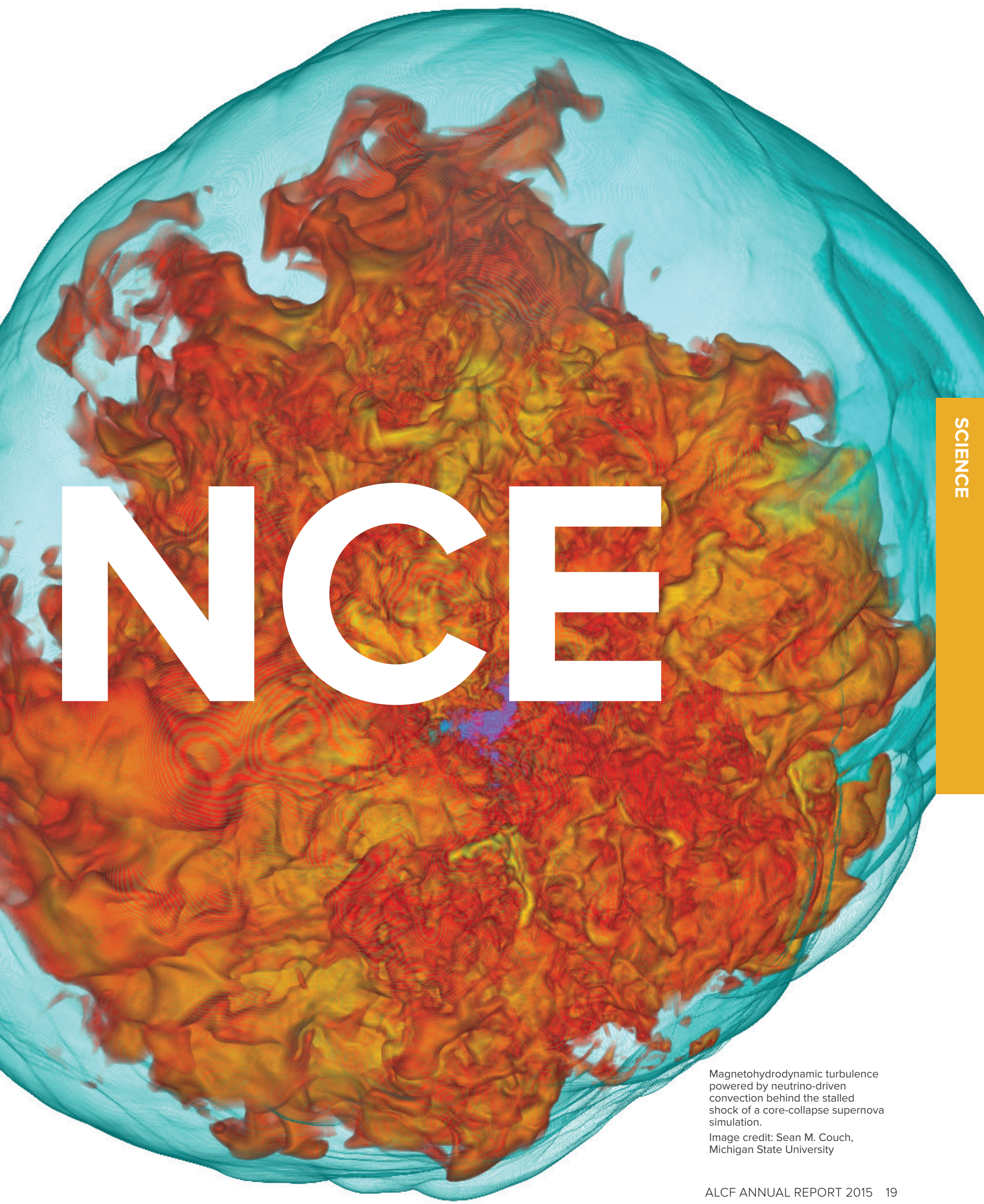




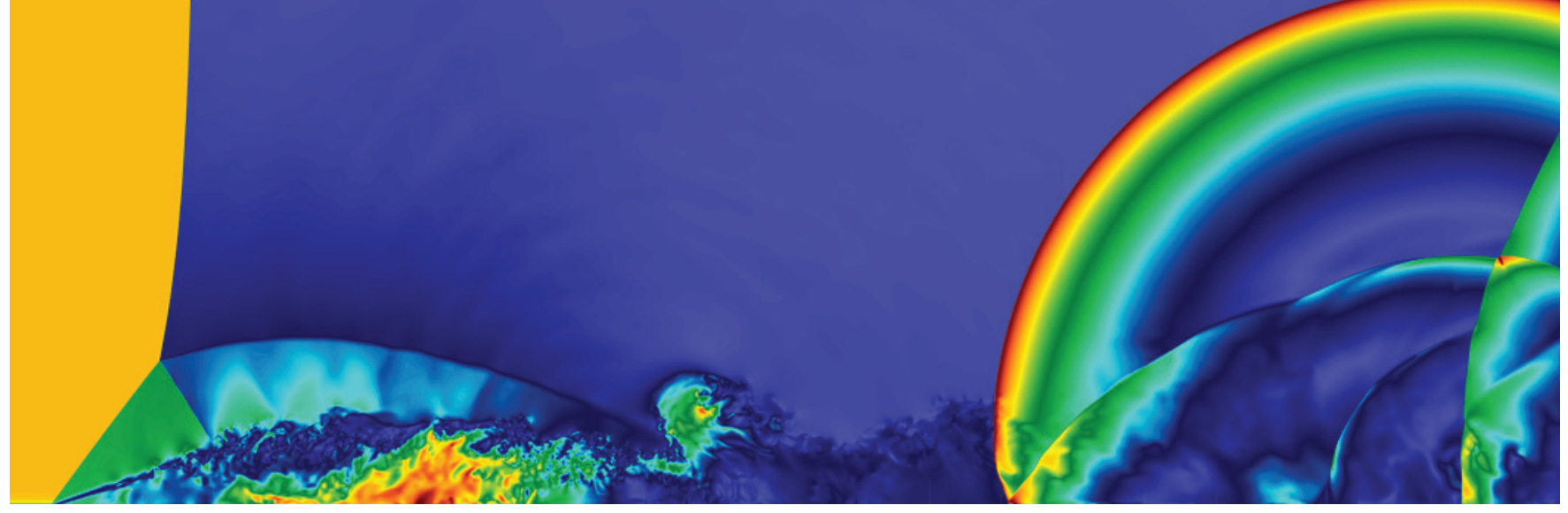

\section{FIRST-PRINCIPLES SIMULATIONS OF}

\section{HIGH-SPEED COMBUSTION AND DETONATION}

\section{ALEXEI KHOKHLOV}

ajk@oddjob.uchicago.edu The University of Chicago

\section{INCITE}

150 Million Core-Hours Chemistry

Image Weak ignition behind a reflected shock in $2 \mathrm{H}_{2}+\mathrm{O}_{2}$ mixture at initially atmospheric pressure. The figure shows a 2D distribution of density in the middle of a shock tube with a square cross-section of $5 \mathrm{~cm}$ $x 5 \mathrm{~cm}$. The end wall of the tube is on the right. The reflected bifurcated shock is on the left and is moving to the left. Weak ignition took place near the end wall approximately 60 microseconds after the shock reflection. The transition of the flame to a detonation happened several microseconds later. Expanding detonation wave is visible on the right.

Image credit: Charles Bacon, Marta García, and Joseph A. Insley, Argonne National Laboratory; Alexei Khokhlov, The University of Chicago; Joanna Austin and Andrew Knisely, University of Illinois at Urbana-Champaign
Detonation waves caused by deflagration-to-detonation transition (DDT) in gaseous mixtures present a significant hazard in the production and delivery of combustible chemicals and fuels. Hydrogen fuel is particularly sensitive to detonation, and DDT hazard is also a potential threat to the safety of certain types of water-cooled nuclear reactors. Because detonation events occur quickly with multiple effects and mechanisms acting concurrently, high-resolution, multidimensional simulations are the most feasible method for investigating the detailed physics of DDT.

\begin{abstract}
APPROACH Researchers from the University of Chicago are using Mira to perform a systematic first-principles study of combustion and detonation phenomena in hydrogen-oxygen mixtures and other reactive gases in various settings. Their study requires using direct numerical simulation (DNS) to explicitly resolve physical processes on spatial scales ranging from meters to microns, as well as attendant shocks, discontinuities, and physical variables. Researchers are performing these simulations with a reactive flow NavierStokes high-speed combustion and detonation code, which incorporates detailed physics and chemistry suitable for hydrogen combustion and highresolution treatment of shock waves. The code also supplies a uniform grid, and static and dynamic adaptive mesh refinement capabilities.
\end{abstract}

RESULTS The research team has conducted the first 3D reactive flow NavierStokes DNS of flame acceleration and DDT in a stoichiometric hydrogen-oxygen mixture at $1 \mathrm{~atm}$ initial pressure in a $2.5 \mathrm{~cm}$ square pipe with smooth and rough walls. They found wall roughness introduces additional turbulence, which leads to a somewhat faster DDT. It also presents a drastic change in the location of the DDT. In smooth tubes, the DDT was observed ahead of the turbulent flame brush, in a hot gas pre-compressed by the rapidly accelerating flame. However, in the case of rough walls, the team found that the DDT occurs inside the flame brush.

IMPACT This project is using advanced simulations to study the fundamental physics of DDT, providing insights that are not observable in experiments. An improved understanding of the complex mechanisms involved in the detonation of reactive gases will help researchers and engineers to better predict the onset of detonation and develop safety mechanisms for real-world applications. 


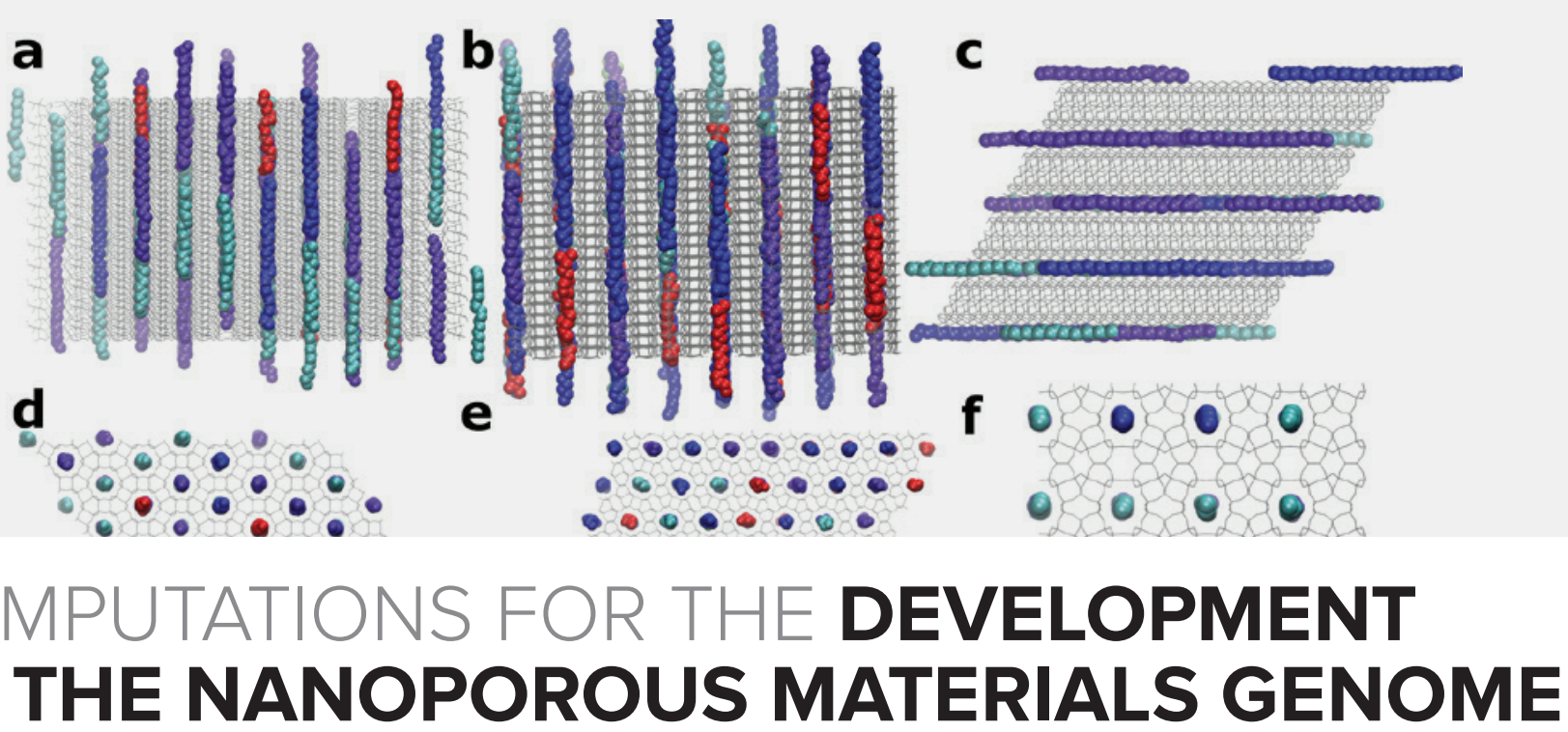

\section{J. ILJA \\ SIEPMANN}

siepmann@umn.edu

University of Minnesota

\section{Director's Discretionary}

8 Million Core-Hours

Materials Science
Image Snapshots of representative hydrocarbon configurations inside zeolite frameworks. Views facing the main channel axis $(a-c)$ and along the main channel axis $(d-f)$ are shown for ATO (a,d), MTW (b,e) and PCOD-8113534 (c,f). Zeolite frameworks are depicted as grey lines, and n-octadecane, n-tetracosane, n-triacontane, (2-methyl and 4-methylheptadecane) and 2,2-dimethylhexadecane molecules as cyan, purple, blue, red and green spheres, respectively. Image credit: Peng Bai and J. Ilja Siepmann, University of Minnesota
In the petrochemical and biofuel industries, aluminosilicate materials called zeolites are used as catalysts and molecular sieves to aid in the processing of fuels and chemical feedstocks. To date, more than $\mathbf{2 0 0}$ types of zeolites have been synthesized and more than 330,000 thermodynamically feasible zeolite structures have been predicted using computational searches. With such a large pool of candidate materials, traditional laboratory methods could take decades to identify the optimal zeolite for a particular application.

\begin{abstract}
APPROACH To help accelerate this process, scientists from the University of Minnesota and Rice University used Mira to demonstrate a predictive theory and modeling tool that can rapidly screen thousands of materials to pinpoint promising candidates for further research and development. ALCF staff helped ensure optimal performance on Mira by guiding the developers of the MCCCSMN code (Monte Carlo for Complex Chemical Systems) in adding OpenMP support to permit hybrid MPI/OpenMP parallelism, and helping design an MPIbased framework to allow high-throughput calculations capable of using all of Mira's 786,432 cores
\end{abstract}

RESULTS The team's computations successfully identified new zeolites for two important applications. One of the zeolites has the ability to purify ethanol from fermentation broths in a single separation step, demonstrating the potential to replace an energy-intensive, multi-step distillation process currently used by industry. To validate the simulation results, the researchers synthesized and tested the promising zeolite, providing experimental data that was in very good agreement with the predictions. For the second application, the team investigated potential zeolite-based catalytic systems for a dewaxing process called hydroisomerization, in which linear long-chain alkanes are transformed into slightly branched alkanes to reduce the pour point and increase the viscosity of lubricant oils and other fuel products. Their simulations identified zeolites with up to 100 times better adsorption capability than current technology used for this process. The research team published results from this project in Nature Communications.

IMPACT With the ability to identify optimal zeolites and metal-organic frameworks for particular tasks, this predictive modeling capability has the potential to benefit the production of biofuel and petroleum products, and the development of gas storage and carbon capture devices, while reducing the time and cost of associated laboratory research and development efforts. 


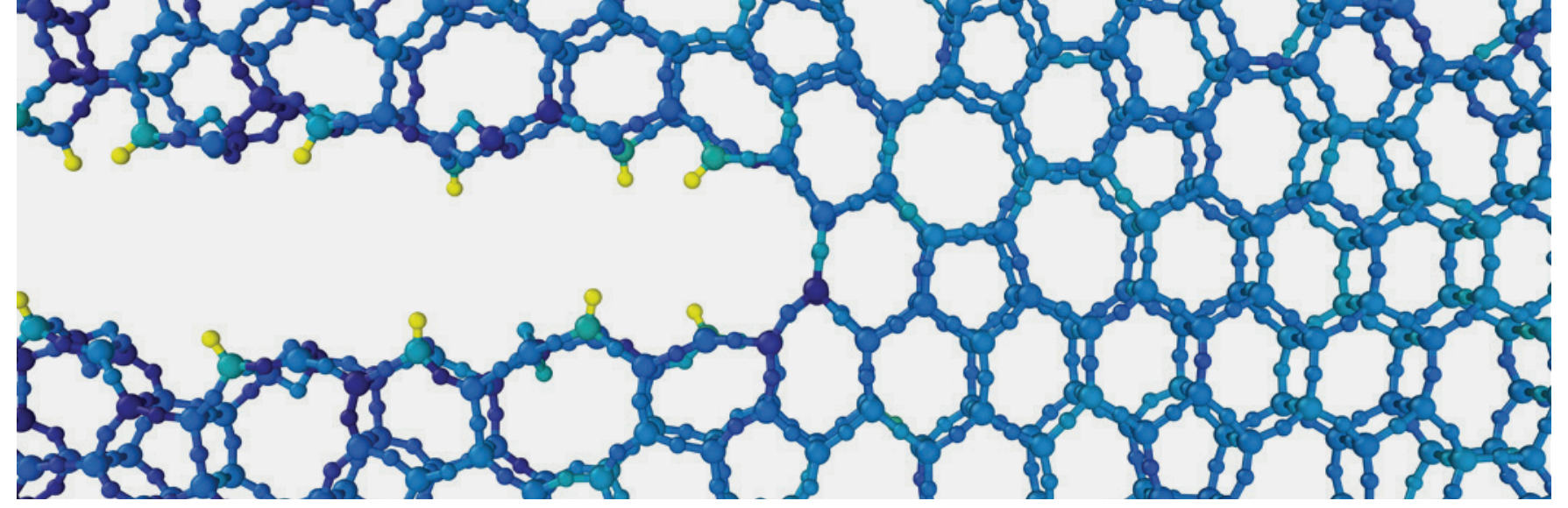

\section{$\mathrm{SiO}_{2}$ FRACTURE: CHEMOMECHANICS WITH A MACHINE-LEARNING HYBRID QM/MM SCHEME}

\section{JAMES KERMODE}

j.r.kermode@warwick.ac.uk University of Warwick

\section{INCITE}

125 Million Core-Hours Materials Science

Image Crack propagation in a twodimensional bilayer of amorphous silicon dioxide modeled with a polarizable interatomic potential. Atoms are colored by their local potential energy on a scale normalized from dark blue, lowest energy, to yellow, highest energy. Image credit: Marco Caccin and Alessandro De Vita, King's College London; James Kermode,

University of Warwick
Fracturing minerals requires a tremendous amount of energy. In mining, the energy spent fracturing represents almost $\mathbf{8 5}$ percent of such activity. The World Business Council estimates that mining is responsible for nearly 5 percent of total human energy consumption, and annually releases millions of tons of carbon dioxide. Understanding the physical process of grinding and crushing minerals under different environments has a technological and scientific relevance to reduce the ecological footprint of mining.

\begin{abstract}
APPROACH A collaborative team from the University of Warwick, King's College London, the University of Basel, and Argonne National Laboratory is using Mira to improve the techniques required to understand the intricate mechanisms of fracturing materials, and to overcome the challenges of simulating large materials and complex physical processes at atomic resolution. The team is employing a quantum mechanical/molecular mechanical (QM/ MM) approach coupled with a Machine Learning on-the-Fly (MLOTF) method, which is of particular interest since it enables the systematic study of large systems with QM precision for the first time. ALCF staff helped the project to move forward by porting the CP2K electronic structure code and enabling an optimized version of the linear algebra library ELPA for large QM simulations.
\end{abstract}

RESULTS The team's work is advancing the techniques required to understand the intricate mechanisms of fracturing materials, and is helping to overcome the challenges of simulating large materials and complex physical processes at atomic resolution. The MLOTF scheme efficiently combines classical and quantum mechanics to study bond breaking in amorphous systems. The researchers have demonstrated that MLOTF works on the fracture of real 3D materials such as silicon and silica, common components in rocks. In practical tests, MLOTF has shown a speedup of up to $10^{3}$ compared to carrying out QM calculations at every time step.

IMPACT This research has implications for the manufacture of silica-based products resistant to fracture, and could significantly reduce energy and cost expenditures associated with mineral ore processing. The methodology used has potential future application in the study of a wide variety of materials and processes. 


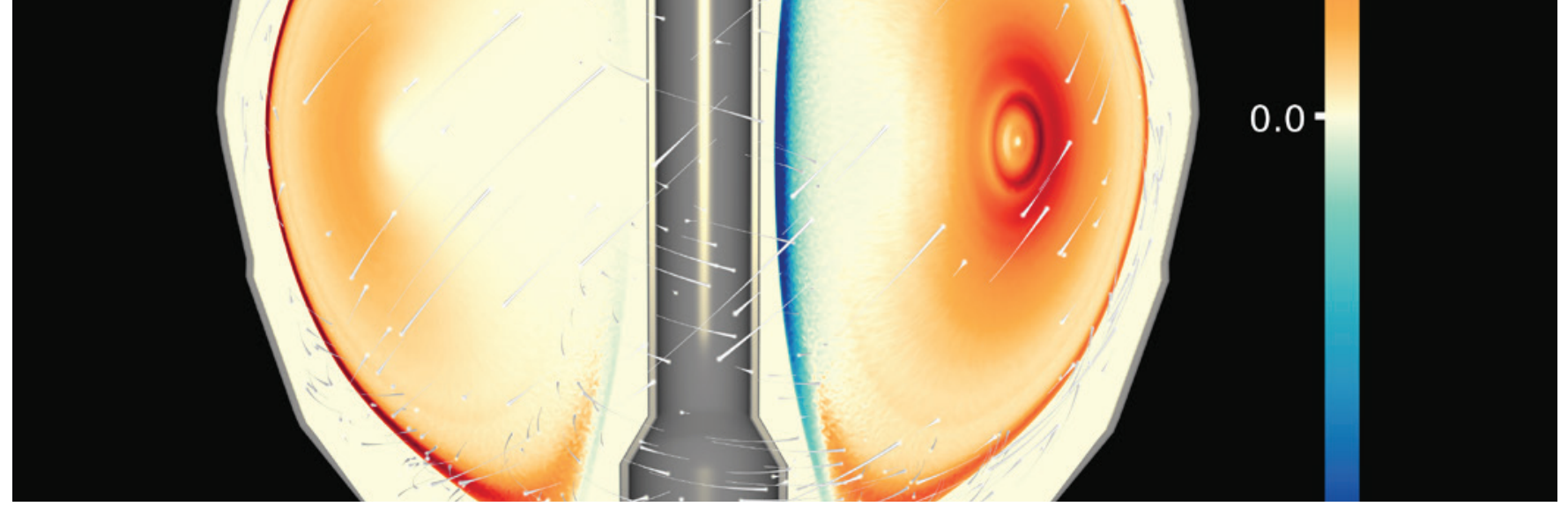

\section{HIGH-FIDELITY SIMULATION OF}

\section{TOKAMAK EDGE PLASMA TRANSPORT}

\section{C.S. \\ CHANG}

cschang@pppl.gov

Princeton Plasma Physics

Laboratory

\section{INCITE}

270 Million Core-Hours (ALCF: 100M; OLCF: 170M) Physics

Image Developed from simulations on Mira, this image shows trapped (left cross-section) and passing (right cross-section) electrons carried in the bootstrap current of a tokamak, which is in contrast to the previous understanding that the bootstrap current is carried by passing particles only. Based on a series of high-resolution simulations of bootstrap current in present-day tokamak geometries, researchers have modified a well-known formula that calculates the value of bootstrap current in order to improve the prediction of fusion efficiency in tokamak reactors.

Image credit: Kwan Liu-Ma, University of California, Davis
With the potential to produce a clean and virtually inexhaustible energy supply, fusion power could be a global solution to future energy demand. But many difficult scientific problems first must be understood and overcome before a functional fusion power plant can be realized. One of the biggest obstacles to building a magnetically confined fusion energy system, such as ITER, is confining the plasma within a practical amount of space. A wellconfined edge plasma forms a steep pedestal and puts the fusion plasma into a high-confinement mode, which allows an efficient fusion burn in the plasma core.

APPROACH Researchers from the Princeton Plasma Physics Laboratory are using XGCa, the latest iteration of the XGC gyrokinetic code, to gain an understanding of the underlying plasma physics that govern edge plasma confinement in fusion reactors. With help from ALCF staff, the researchers were able to run simulations with XGCa on more than 260,000 cores with excellent scalability, allowing the team to accurately evaluate a strong self-generated electrical current, called "bootstrap current," that runs through the plasma. This current helps enable better confinement of the edge plasma, and therefore higher fusion efficiency.

RESULTS The team's simulations revealed electron behaviors related to bootstrap current that are not accurately predicted for present-day tokamak geometry by the Sauter formula, which is used to calculate values for the bootstrap current. Two types of electrons characterize bootstrap current: passing and trapped. Based on tokamak geometries with large aspect ratios, physicists have understood for decades that passing electrons in the core plasma are primarily responsible for carrying the bootstrap current. However, with more detailed simulations of trapped and passing electrons of the edge plasma, the INCITE researchers observed that trapped electrons can take on the responsibility of carrying bootstrap current at the edge where there are fewer passing electrons. Numerous simulations for different geometric and plasma conditions led to a new unified analytical formula that can describe the edge current, as well as the core current.

IMPACT This new analytical formula is expected to be widely used by international fusion researchers studying edge plasma physics. This will enable the fusion research community to better understand and predict edge plasma behavior, and to ensure highly efficient tokamak performance and more economical ITER operation 


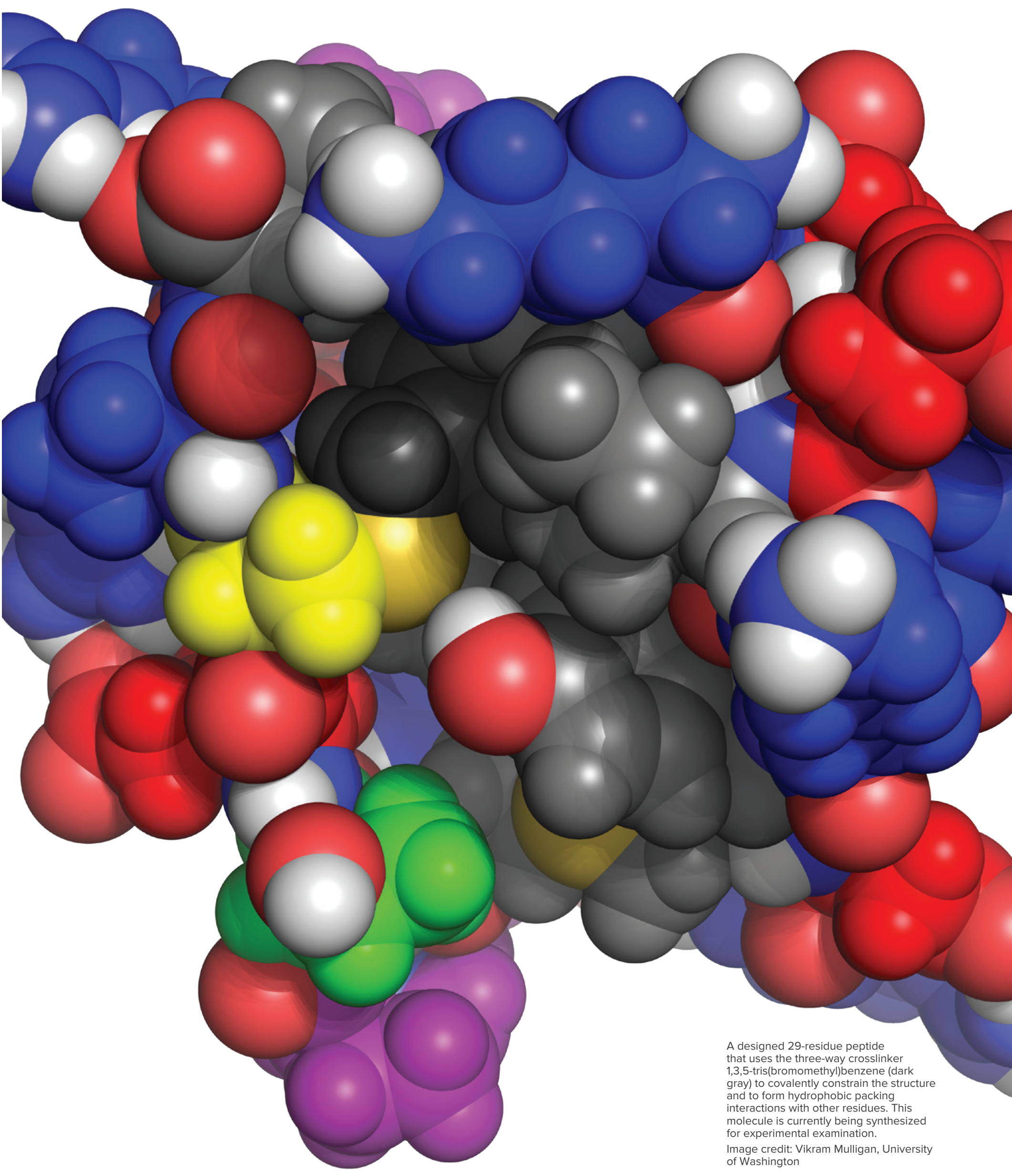



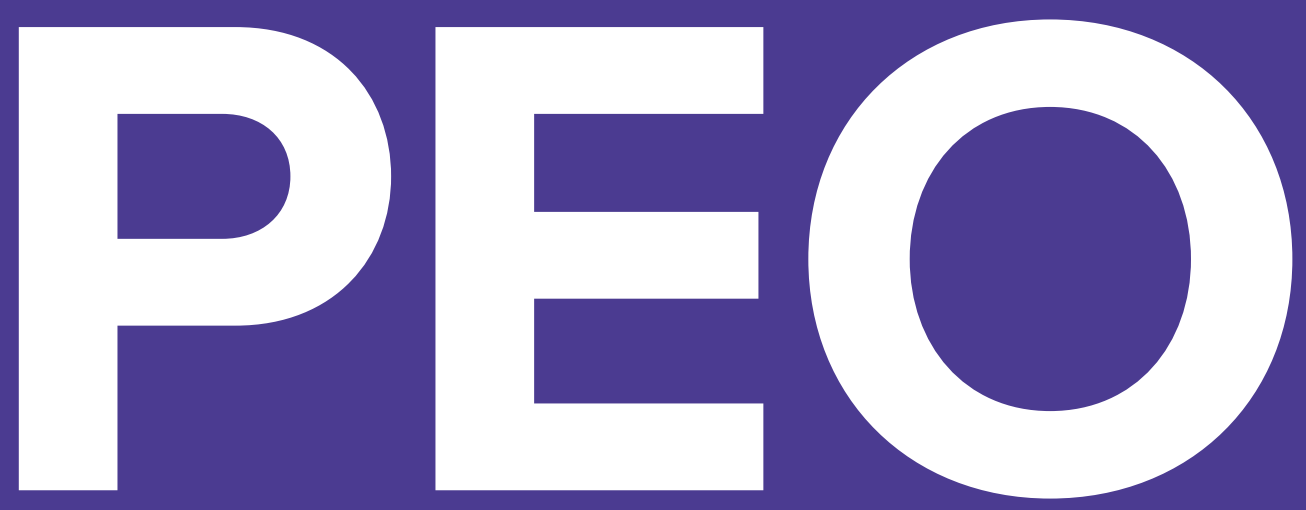

With a shared passion for research and innovation, the ALCF's talented and diverse staff help make the facility one of the world's premier centers for computational science and engineering. 


\section{ALCF EXPERTISE}

\section{The ALCF has assembled a world-class team of experts to help maximize the use of ALCF computing systems.}

\section{Catalysts}

Catalysts are computational scientists who work directly with project teams to maximize and accelerate their research efforts. With multidisciplinary domain expertise, a deep knowledge of the ALCF computing environment, and experience with programming methods and community codes, the team helps users surmount any obstacles they may encounter using ALCF computing resources. This includes collaborating with colleagues from other ALCF teams to resolve issues when additional expertise is required.

\section{Data Analytics and Visualization}

Data Analytics and Visualization experts facilitate the use of tools and methods for high-performance post processing of large datasets, interactive data exploration, batch visualization, and production visualization.

\section{Operations}

Operations ensures that system hardware and software work reliably and optimally; system tools are matched to the unique system architectures and scale of ALCF resources; the entire system software stack works smoothly together; and I/O performance issues, bug fixes, and requests for system software are addressed.

\section{Performance Engineers}

Performance Engineers help users achieve optimal performance on ALCF resources by working with them to port, tune, and parallelize scientific applications and other software on current and emerging systems. The team also helps drive the future of high-performance computing and data-intensive computing through research and development of performance models and scalable algorithms, and the development of standards for key areas, such as benchmarks, compilers, and programming models.

\section{User Experience}

User Experience coordinates communications, support, and outreach for the ALCF. The communications team stewards ALCF user stories and ensures that the facility provides relevant and accurate information to users, DOE, and the public at large. The support team manages the workflows for user accounts and projects, and assists users with support requests related to their ALCF projects. Outreach focuses on organizing training efforts and user-centered events for the ALCF. 


\section{ALCF WELCOMES TWO NEW DIRECTORS}

\section{The ALCF filled two important leadership positions in 2015.}

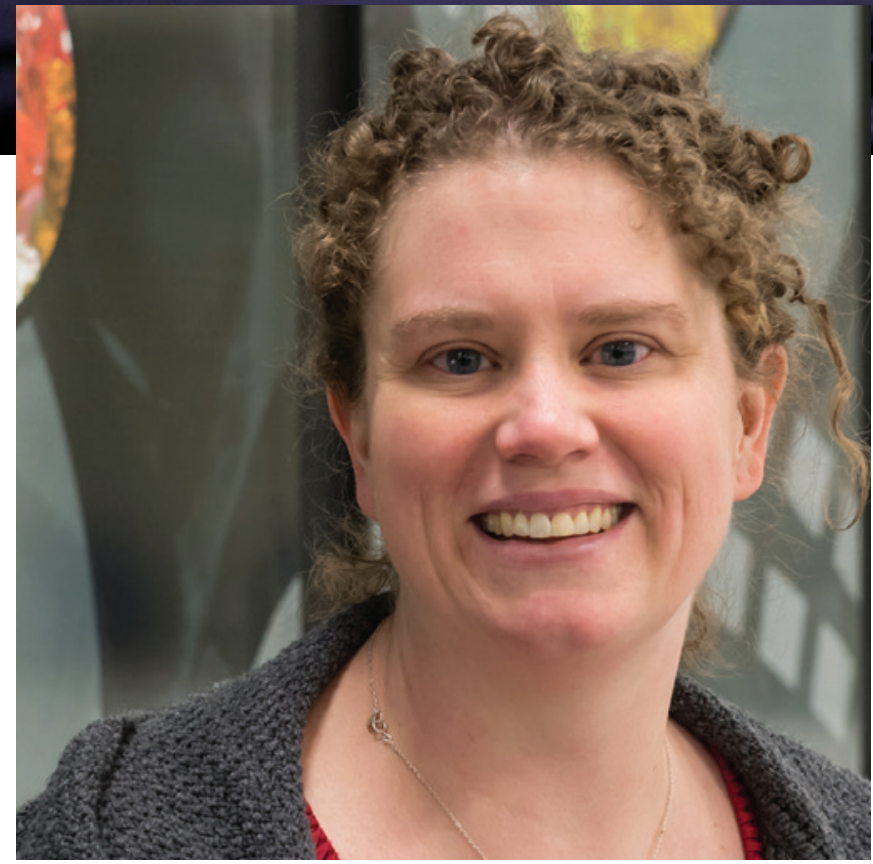

Katherine cultivates and guides teams of scientists and engineers to use one of the most powerful supercomputers in the world dedicated to open science.

\section{KATHERINE RILEY}

\section{Director of Science}

The ALCF is familiar territory for Katherine as she was one of the facility's first hires in 2007. In her current role as Director of Science, she leads scientific strategy for the ALCF, ensuring the facility delivers leading-edge computational capabilities and expertise that help advance fundamental discovery and understanding in a broad range of scientific and engineering disciplines.

\section{Katherine has spent her career focused on} scientific application architecture and how it impacts performance, scalability, and extensibility. She previously served as a principal scientific applications engineer and manager of the ALCF catalyst team, a group of computational scientists who work directly with users to help them maximize their time on ALCF systems and achieve their research goals. Katherine has also been a key contributor to the strategic vision of the facility, assisting with the design and development of ALCF supercomputers by helping to identify the scientific requirements for new systems.

Prior to joining the ALCF, she served as scientific applications engineer in the Mathematics and Computer Science Division at Argonne and as a senior scientific programmer at the University of Chicago. 


\section{STAFF SPOTLIGHTS}

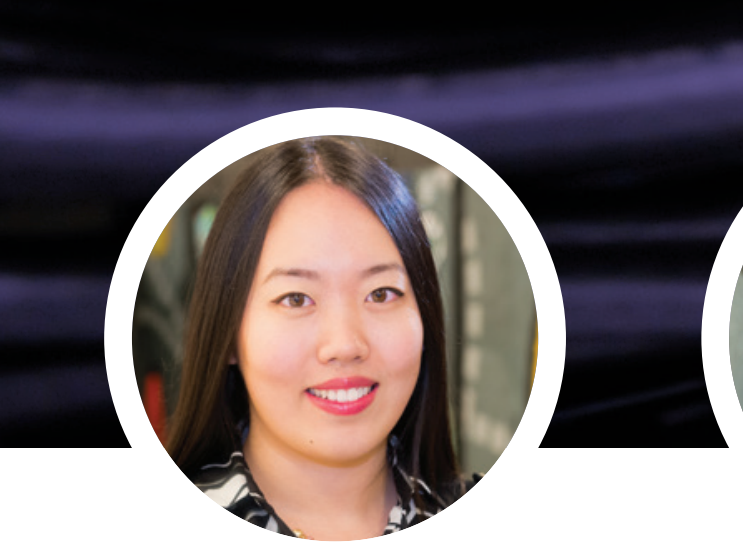

\section{Savannah (Xiao) French UX Developer}

While pursing her degrees in actuarial science and computer science at North Central College, Savannah landed an internship as a research aide at the ALCF. Her first task was to design and build an interactive website graphic that displayed real-time information about the jobs running on Mira. In 2015, Savannah was hired as a full-time developer on the user experience team. In addition to helping maintain the ALCF website, she designs and develops research and event websites for strategic initiatives. Savannah was also a key part of the team that executed the ALCF's annual user survey, participating in every step of the process from reviewing survey questions and following up with users to analyzing the final results. The team's efforts resulted in the survey's highest response rate ever.

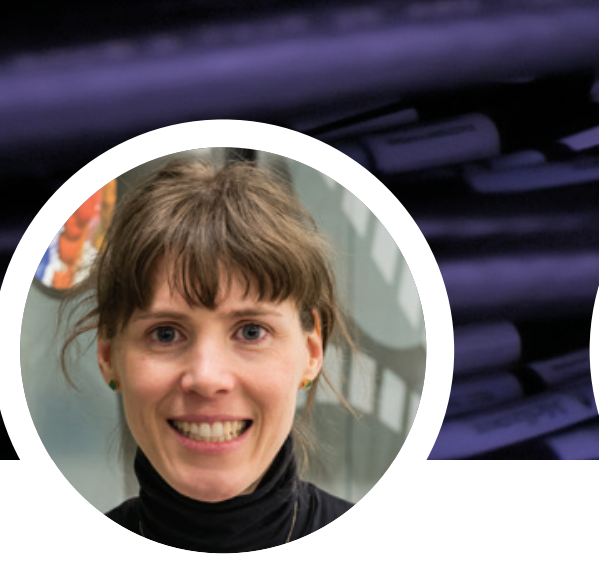

\section{Marta García \\ Computational Scientist}

As a member of the catalyst team, Marta works closely with science teams to maximize their use of ALCF computing resources. Her expertise in computational fluid dynamics has made her a key collaborator on several projects, including work to develop advanced engine modeling and simulation capabilities. In 2015 , Marta played an important role in helping Argonne build its future R\&D programs and capabilities, chairing the Director's Review Committee for Competitive Grants for the Laboratory Directed Research and Development (LDRD) Program. She was also one of four Argonne researchers featured in the "Catch a Rising Science Star" public lecture, which showcased some of Argonne's best and brightest young scientists. In 2016, Marta will take the reins from former ALCF Director of Science Paul Messina as the program manager of the Argonne Training Program on Extreme-Scale Computing.

\section{Christopher Knight Computational Scientist}

Chris served as a guest lecturer at Harvard University this fall, leading six sessions as part of a graduate-level course on extreme-scale computing. The hands-on lessons, which included running jobs on Mira, helped the students (most of whom did not have a background in science) understand how supercomputers can be used to address real-world problems, such as designing better batteries. At the ALCF, Chris's recent work with a University of Minnesota team resulted in a Nature Communications paper that discussed their promising results with a predictive modeling tool for identifying optimal zeolites. He is now co-PI for the team's ALCC project focused on modeling functional nanoporous materials. Chris is also working closely with an Argonne/University of Chicago team investigating materials for energy conversion and storage, serving as co-PI of an ALCC award and a collaborator for their Theta ESP project. 


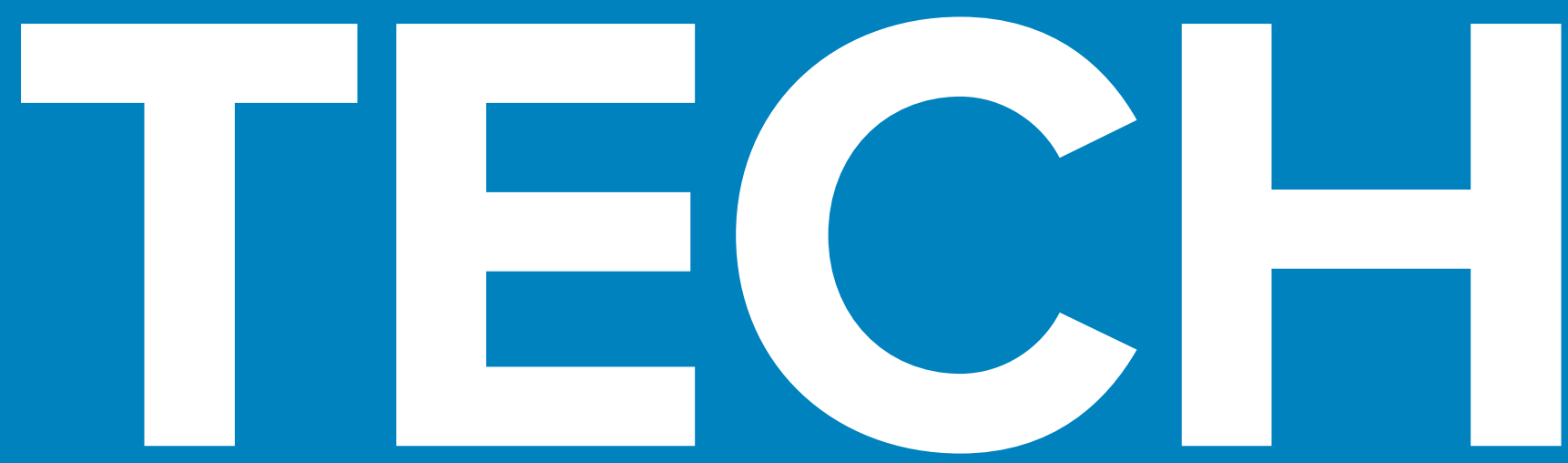

ALCF researchers are helping to develop the technologies needed to drive continued improvements in supercomputing performance. 


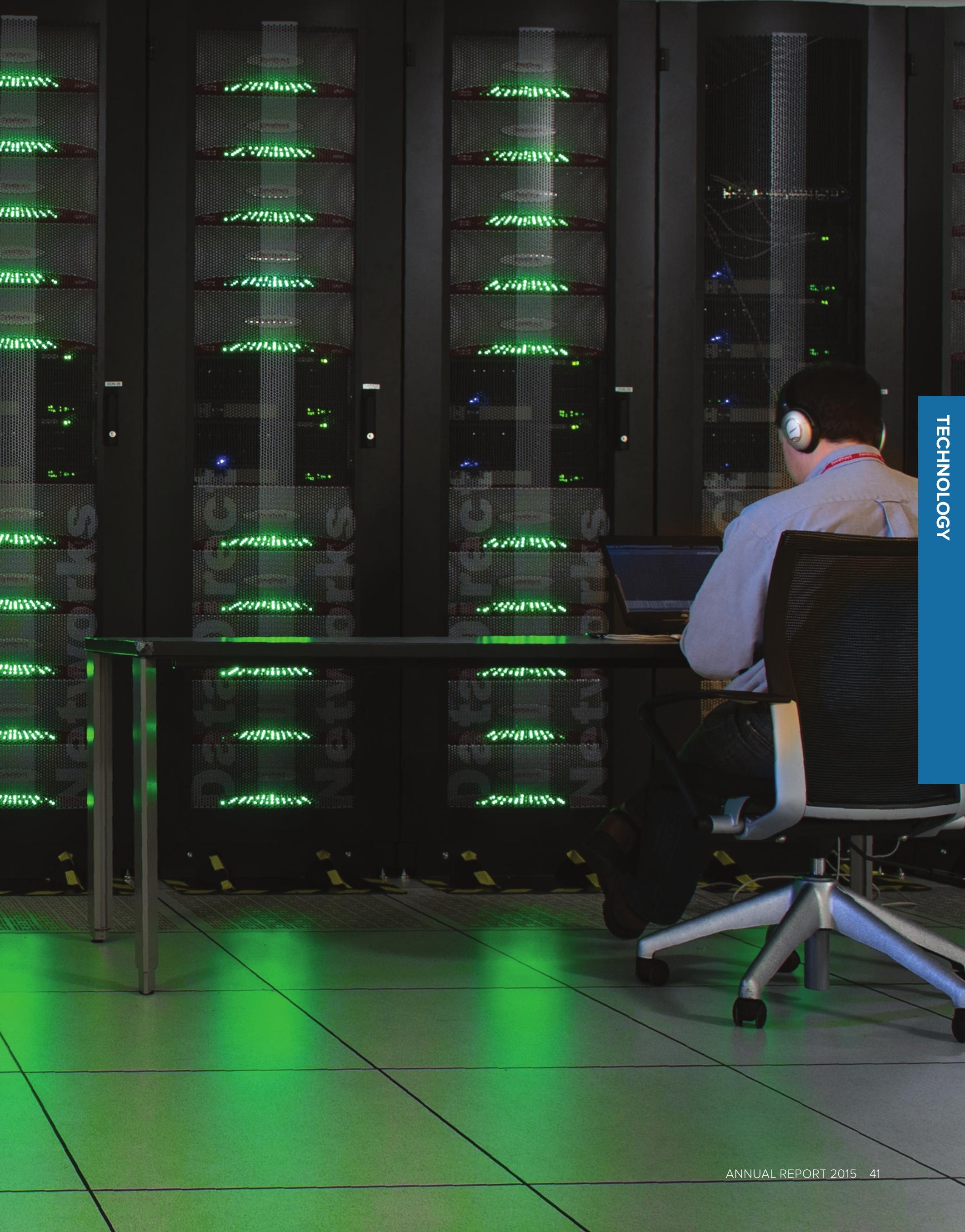




\title{
ENABLING SCIENCE THROUGH HPC TECHNOLOGY
}

\author{
ALCF researchers are developing software tools \\ and approaches that are helping scientific applications \\ run more efficiently on its leadership-class systems.
}

\begin{abstract}
AutoPerf
Launched in 2015, AutoPerf is an ALCF-developed library that automatically collects performance information for applications running on ALCF systems. The tool records data from simulations and saves it into files for analysis when the job is completed. Output includes MPI usage and performance information that indicates which MPI routines were called, how many times each routine was called, the time spent in each routine, the number of bytes sent or received (if applicable), and data from the systems' hardware performance counters. This information will help ALCF staff better understand the requirements of applications and how to optimize their performance.
\end{abstract}

\section{Compiler and Library Tracking}

The ALCF deployed two software tools in 2015 to track the compilers and libraries being used on its systems. The first stage in the librarytracking process is a component called Trackdeps, which records paths to all inputs to the build process that contribute to the final output, including compiler identity, header files, Fortran module files, and libraries. Tracklib, the second stage in the librarytracking process, is a set of tools used to examine programs as they run on Mira, producing the data necessary to match the job's accounting information with the data collected by Trackdeps. With these tools in place, staff can determine how various libraries and compilers are being used at the facility, providing knowledge that helps inform support and research priorities for the ALCF and DOE.

\section{Darshan}

ALCF researchers are collaborating with staff from Argonne's Mathematics and Computer Science Division on the continued development of Darshan, a scalable HPC I/O instrumentation library designed to capture an accurate picture of application I/O behavior. This tool records statistics such as the number of files opened, time spent performing $\mathrm{I} / \mathrm{O}$, and the amount of data accessed by an application. Darshan's lightweight design makes it suitable for full-time deployment for workload characterization of large systems. The information revealed by Darshan enables researchers to investigate and tune the $\mathrm{I} / \mathrm{O}$ behavior of complex HPC applications, while also helping the storage research community to develop strategies and approaches to better serve the needs of scientific computing. Darshan was originally developed on the IBM Blue Gene series of computers at the ALCF, but it is portable across a wide variety of platforms and is now deployed at multiple DOE computing facilities including NERSC, OLCF, and Lawrence Livermore National Laboratory. 


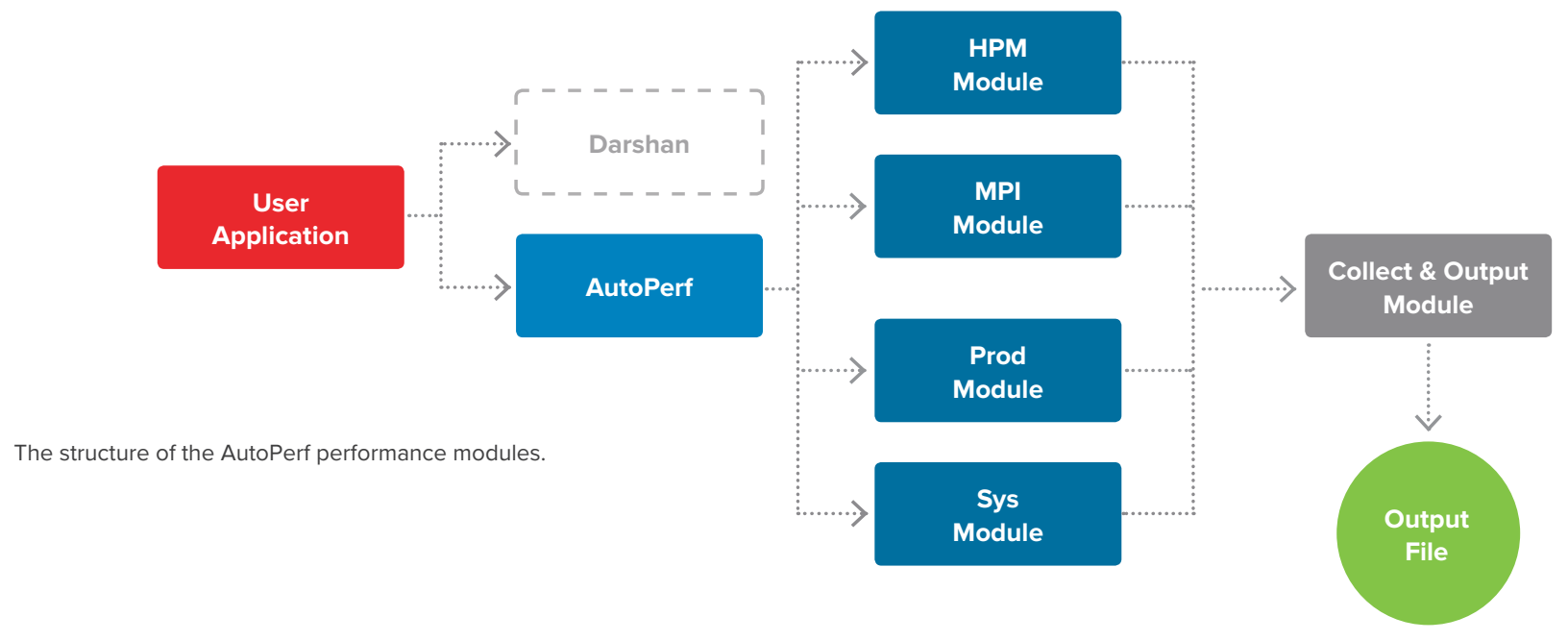

\section{Cobalt}

Developed at Argonne, Cobalt is the ALCF's job scheduler and resource manager. This tool gives the facility great flexibility in executing its scheduling policies, accommodates diverse workloads, and supports important features (e.g., alternate kernel support) that are either not supported or poorly supported by other schedulers and resource managers. The ALCF continues to develop and improve Cobalt to meet future resource scheduling needs. In 2015, ALCF researchers began preparing Cobalt to support its next-generation systems, Theta and Aurora, when they are deployed. The Cobalt development team also implemented a draining and backfilling scheme for cluster systems, like Cooley, which improves utilization of the resource and prevents excessive delays in the start of jobs with larger node counts.

\section{GLEAN}

ALCF researchers are developing an open source software tool called GLEAN to help users optimize data movement between the compute, analysis, and storage resources of HPC systems. GLEAN improves I/O performance by exploiting the network topology for collective $\mathrm{I} O$, leveraging data semantics of applications, and incorporating asynchronous data staging. GLEAN can also be used to perform in-situ analysis during large-scale simulation runs, which allows researchers to analyze data in real time. Algorithms and components of GLEAN are being used by projects running on ALCF systems, and are being integrated with the IBM system software stack to benefit users of IBM Blue Gene/Q systems.

\section{Optimal Scheduling of In-Situ Analysis}

With in-situ analysis, researchers can perform real-time data analysis and visualization during large-scale simulation runs. To help facilitate this capability, ALCF researchers are investigating the challenge of scheduling the analyses with the simulation. The team used a mixedinteger linear program to maximize the number of online analyses subject to resource constraints, such as I/O bandwidth, network bandwidth, rate of computation, and available memory. Using Mira, they performed case studies with the LAMMPS and FLASH codes that demonstrated the effectiveness of their approach. The results were presented at SC15.

The team plans to extend this work by optimally scheduling the analyses computations on different resources. 


\section{NEW SYSTEMS PROVIDE ADVANCED CAPABILITIES}

\section{Leading-edge computing technologies are intrinsic to the ALCF. The facility is continually developing and deploying resources that support forefront computational research.}

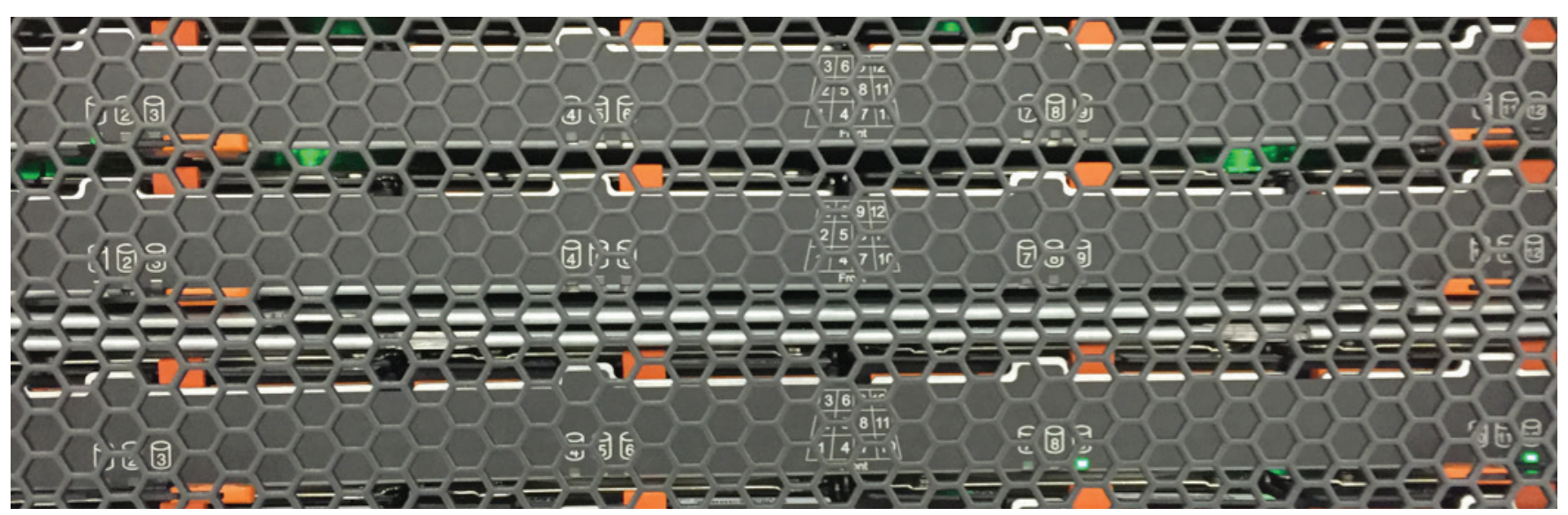

\section{Cooley}

In June, the ALCF launched Cooley, a new visualization and analysis cluster with nearly eight times the memory capacity of the facility's previous system, Tukey. This significant memory boost, along with Cooley's state-of-the-art hardware, is helping ALCF users to better analyze and explore the massive datasets that result from their simulations on Mira. Some of the new and improved capabilities enabled by Cooley include: in-situ analysis, volumerendered visualizations, meshing complex geometries, and uncertainty quantification analysis.

\section{Storage Upgrade}

The ALCF is in the midst of a storage infrastructure upgrade aimed at reducing the amount of time users have to spend on data management and I/O efforts. In 2014, the operations team added a second IBM General Parallel File System (GPFS) to compliment its primary GPFS. This year, they continued work on a burst buffer-like storage cache that is being developed to provide users with much faster I/O rates for running jobs. The team is also working on a service to automatically archive data to longterm storage. In addition, they tripled the facility's archival storage capacity by replacing its LTO4 tape drives with LTO6 tape drives.

\section{Petrel}

Researchers from ALCF and Globus are collaborating to develop Petrel, a pilot data service that allows researchers to store and share largescale datasets with collaborators without the burden of local account management. Petrel leverages the ALCF's storage and infrastructure and Globus's transfer and sharing services to provide a mechanism to store large research data, move data in and out of the system, and make subsets of the data available to collaborators. Petrel has been deployed for researchers at Argonne's Advanced Photon Source and will be expanded to other ALCF users in the near future. 


\section{ALCF COMPUTING RESOURCES}

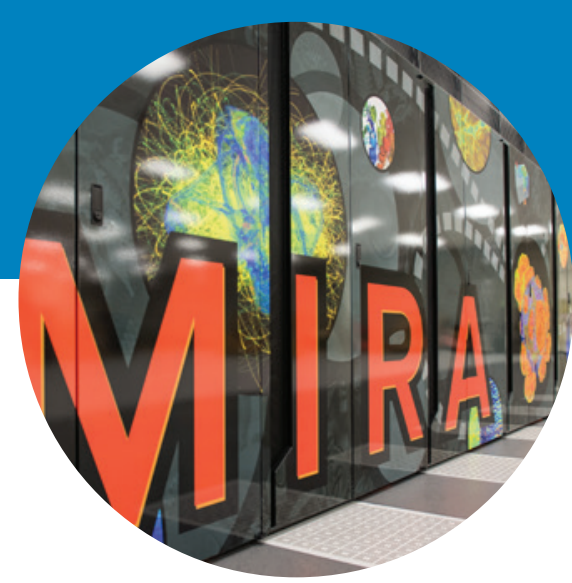

\section{MIRA}

Mira, the ALCF's 10-petaflops IBM Blue Gene/Q supercomputer, opens the door for scientists to analyze data more efficiently, design products more quickly, and address some of society's biggest problems in ways that would otherwise be impossible. The system is capable of carrying out 10 quadrillion calculations per second. Mira is also among the most energy-efficient supercomputers, saving considerable energy through innovative chip designs and a unique water-cooling system.

\section{$\square 48$ racks}

$\square 161600 \mathrm{MHz}$ PowerPC A2 cores per node

$\square$ 49,152 nodes

$\square 786,432$ cores

$\square 768$ TiB RAM

$\square$ 5D torus interconnect

$\square 384 \mathrm{I} / \mathrm{O}$ nodes

$\square$ Peak performance of 10 petaflops

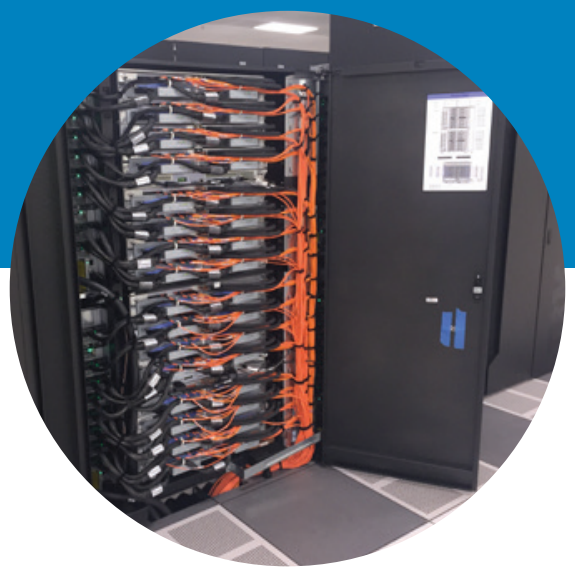

CETUS

The primary role of Cetus is to run small jobs to debug problems that occur on Mira. Cetus shares the same software environment and file systems as Mira.

$\square 4$ racks

$\square$ 4,096 nodes

$\square 64$ TB RAM

$\square$ 5D torus interconnect

$\square 32$ 1/O nodes

$\square$ Peak performance of 838 teraflops

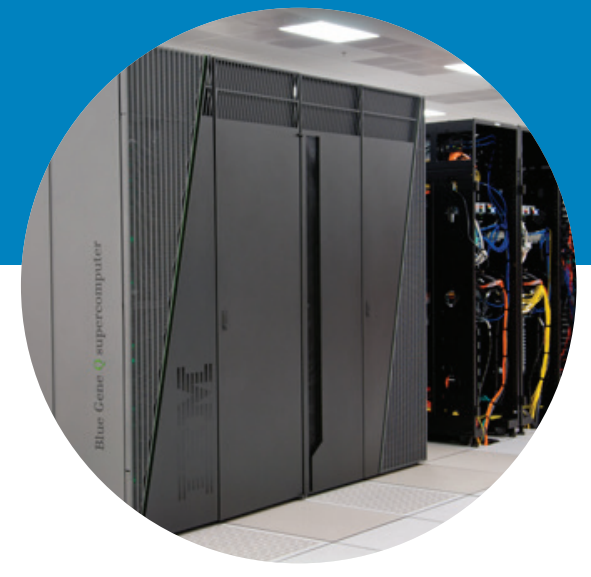

VESTA

As the ALCF's test and development platform, Vesta serves as a launching pad for researchers preparing to use Mira. Vesta has the same architecture as Mira, but on a much smaller scale.

$\square 2$ racks

$\square$ 2,048 nodes

$\square 32$ TB RAM

$\square$ 5D torus interconnect

$\square 32 \mathrm{l} / \mathrm{O}$ nodes

$\square$ Peak performance of 419 teraflops

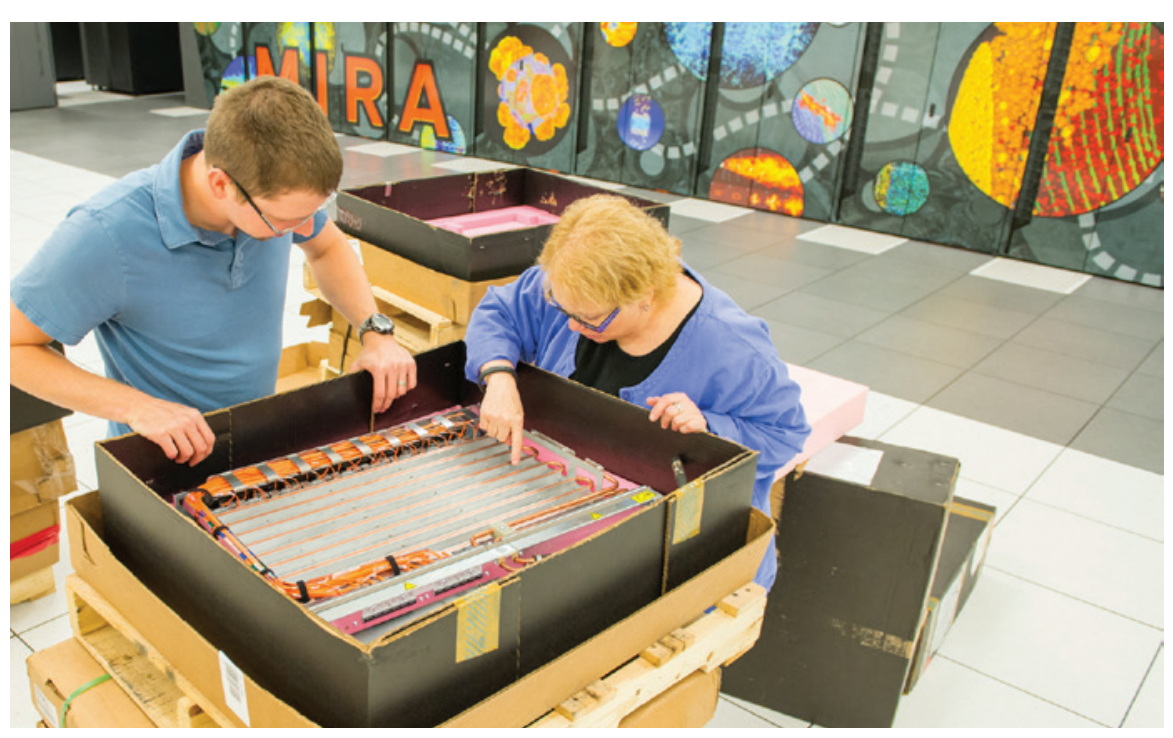

Susan Coghlan and Eric Pershey of ALCF discuss computing hardware in the facility's machine room. 


\section{ALCF users have access to several computing resources, including one of the most powerful supercomputers in the world.}

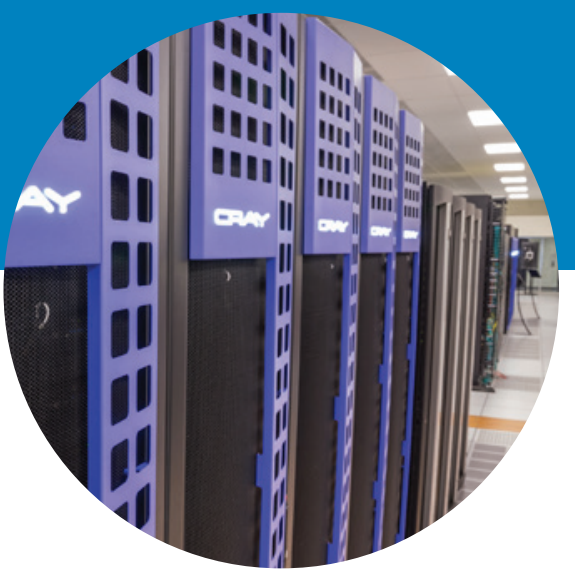

\section{COOLEY}

Cooley is the ALCF's analysis and visualization cluster. Equipped with graphics processing units (GPUs), Cooley converts computational data from Mira into high-resolution visual representations. The resulting images and videos help users to better analyze and understand the data generated by Mira. Cooley can also be used for statistical analysis, helping to pinpoint trends in the simulation data. Additionally, the system is capable of preprocessing efforts, such as meshing, to assist users preparing for Mira simulations. Cooley shares file systems with Mira, enabling direct access to Mira-generated results.

\section{Each Cooley node has:}

$\square 22.4 \mathrm{GHz}$ Intel Haswell E5-2620 v3 6-core processors

$\square$ NVIDIA Tesla K80 GPU accelerator containing two Kepler GK210 GPUs

$\square 384$ GB RAM

$\square 24$ GB GPU RAM

The full Cooley system has:

$\square 126$ nodes

$\square$ 1,512 cores

$\square$ FDR Infiniband interconnect

$\square 47$ TB RAM

๑ 3 TB GPU RAM

$\square$ Peak performance of 293 teraflops

\section{DATA STORAGE}

At the ALCF, disk storage provides intermediate-term storage for active projects, offering a means to access, analyze, and share simulation results. Tape storage is used to archive data from completed projects.

DISK STORAGE The Blue Gene/Q data systems consist of 384 I/O nodes that connect to 22 storage arrays that control 13,000 disk drives with a total useable capacity of 27 PB and a maximum aggregate transfer speed of $330 \mathrm{~GB} / \mathrm{s}$ over two file systems. The ALCF uses the General Parallel File System to access the storage.

TAPE STORAGE The ALCF has two 10,000-slot libraries using LTO 6 tape technology. The LTO tape drives have built-in hardware compression with compression ratios typically between 1.25:1 and 2:1, depending on the data, giving an effective capacity of 26-40 PB.

\section{NETWORKING}

The Blue Gene/Q systems have an internal proprietary network for communicating between nodes. InfiniBand enables communication between the I/O nodes and the storage system. Ethernet is used for external user access, and for maintenance and management of the systems.

The ALCF connects to other research institutions using up to 100 $\mathrm{Gb} / \mathrm{s}$ of network connectivity. Scientists can transfer datasets to and from other institutions over fast research networks, such as the Energy Science Network (ESNet) and Internet2. 
The ALCF is actively engaged in several forward-looking activities that are helping to shape the future of supercomputing.
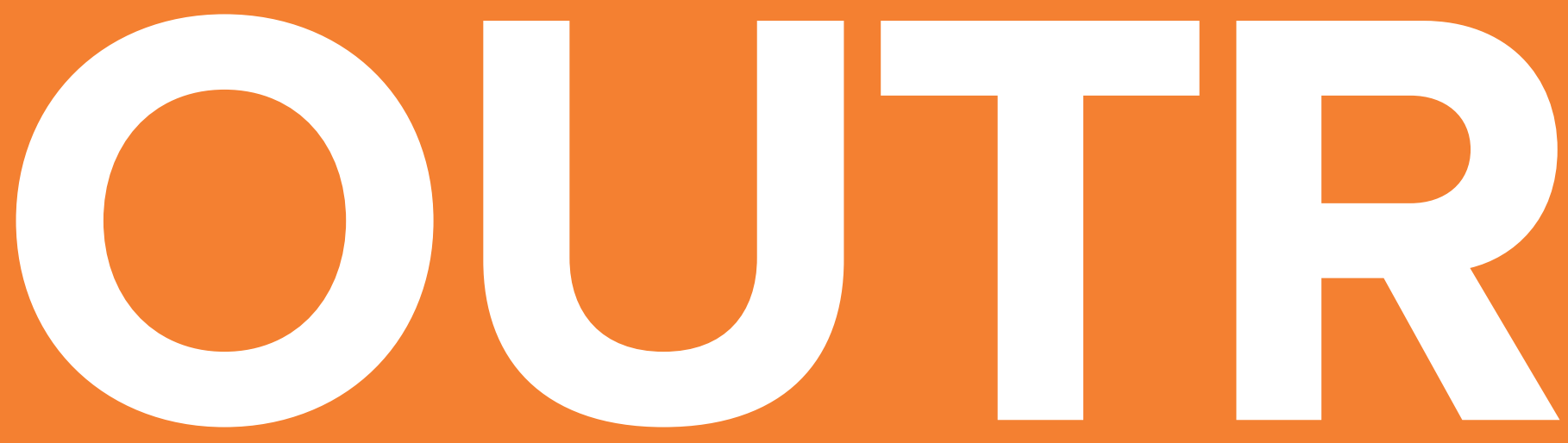


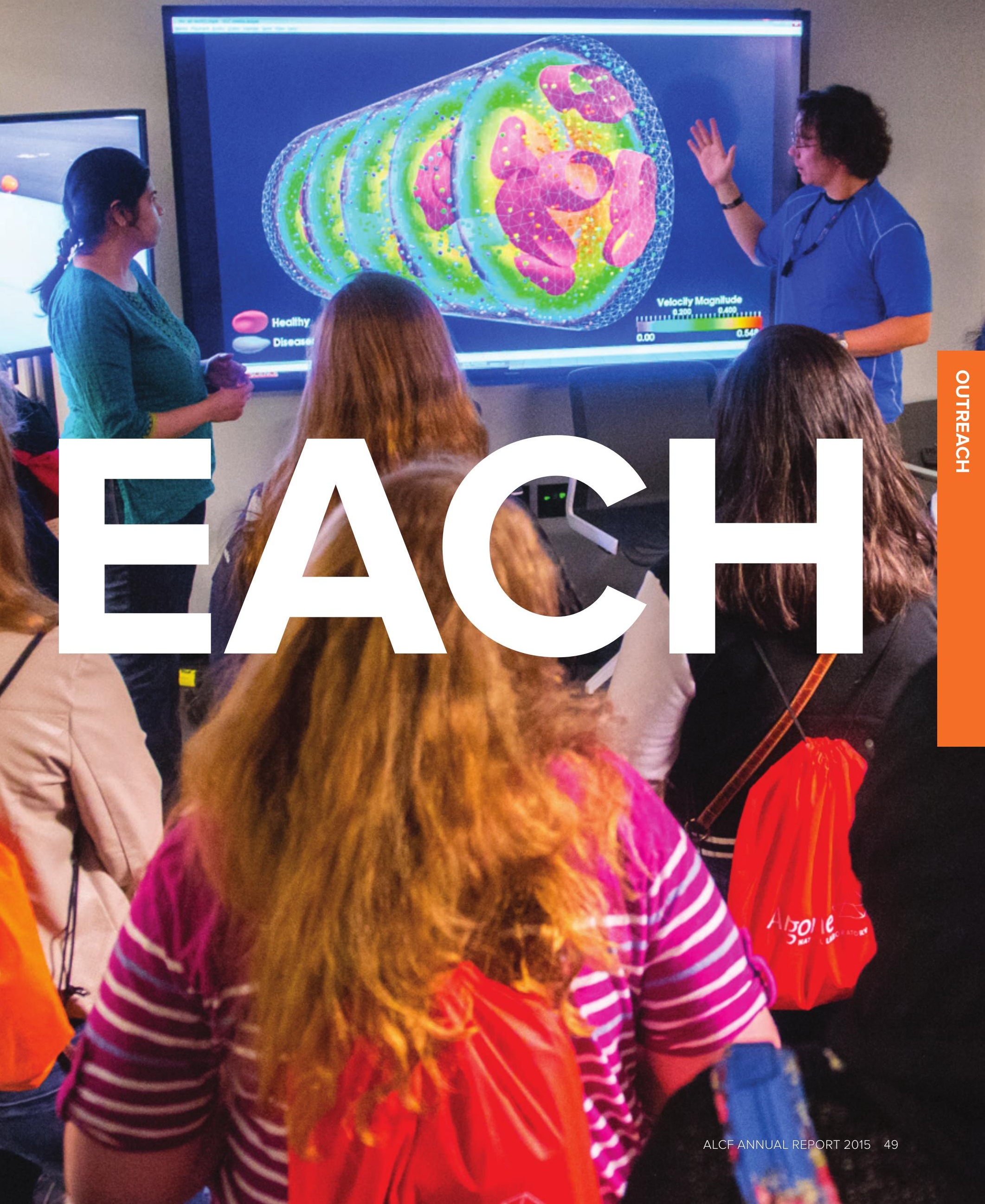


THE HPC COMMUNITY

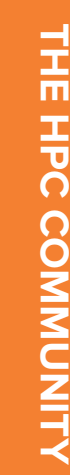

SHAPING JHE
SUPERCOMPUTING
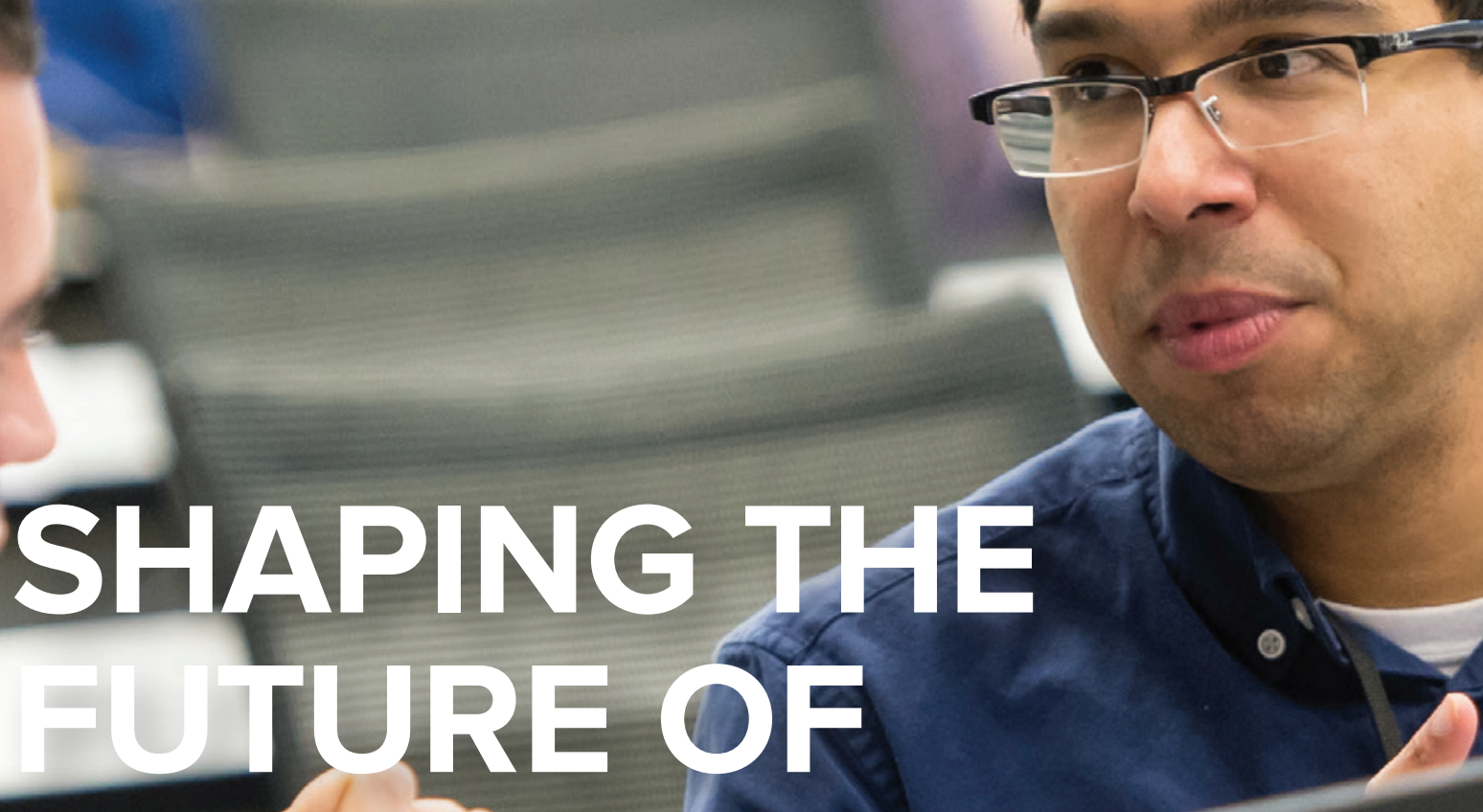


\section{As active members of the HPC community, ALCF researchers lead and participate in several strategic activities that aim to push the boundaries of what's possible in computational science and engineering.}

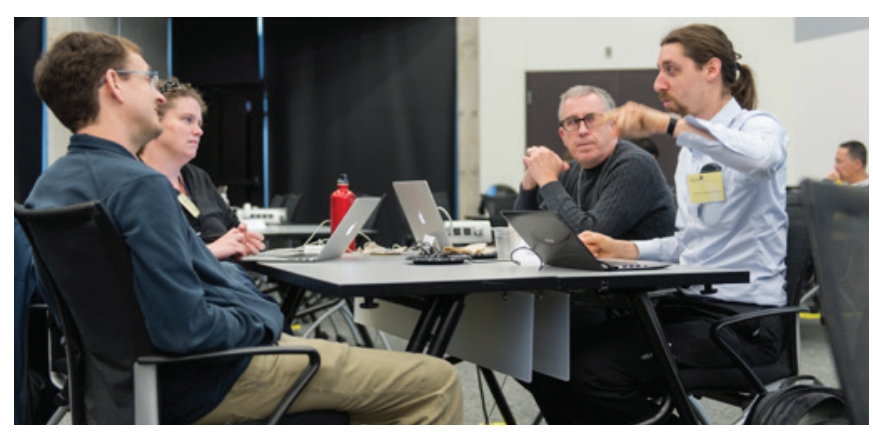

ALCF researchers collaborate to help users prepare for the scale and architecture of leadership computing systems.

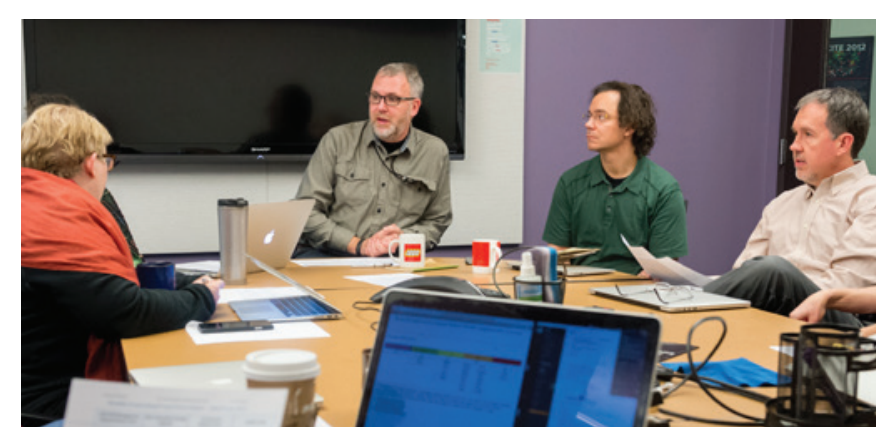

ALCF leadership holds regular meetings to discuss the facility's strategic initiatives.

\section{HPCOR}

ALCF staff played a key role in DOE's High-Performance Computing Operational Review (HPCOR) on Scientific Software Architecture for Portability and Performance in September. Chaired by ALCF Director of Science Katherine Riley, the three-day workshop brought together application developers, computing facilities, vendors, and library and tool developers to identify approaches and best practices for increasing application portability and performance on diverse architectures in the coming years.

\section{Exascale \\ Requirements Reviews}

ALCF is collaborating with the other DOE ASCR facilities, OLCF and NERSC, to hold a series of Exascale Requirements Reviews to determine the mission-critical computational science objectives for each of the six DOE Office of Science program offices through 2025. These workshops bring together key domain scientists and computational science experts to identify the requirements for developing an exascale ecosystem that includes computation, data analysis, software, workflows, HPC services, and other features. The reviews for High Energy Physics and Basic Energy Sciences were held in 2015, with the four remaining reviews planned for 2016.

\section{IXPUG}

The ALCF's next supercomputers, Aurora and Theta, will be based on successive generations of the Intel Xeon Phi processor. As one of the founding members of the Intel Xeon Phi User's Group (IXPUG), the ALCF has been active in the group's mission to provide a forum for exchanging information to enhance the usability and efficiency of scientific and technical applications running on large-scale HPC systems that use the Xeon Phi processor. ALCF Industry Outreach Lead David Martin represents the facility as member of the IXPUG Steering Committee. In 2015, the committee organized a workshop and a birds-of-a-feather (BOF) session at ISC'15, a BOF session at SC15, and the IXPUG Annual Meeting at Lawrence Berkeley National Laboratory. 
The ALCF is committed to providing training and outreach opportunities that prepare researchers to efficiently use its leadership computing systems, while also cultivating a diverse and skilled HPC workforce for the future.

\section{GROWING THE HPC COMMUNITY

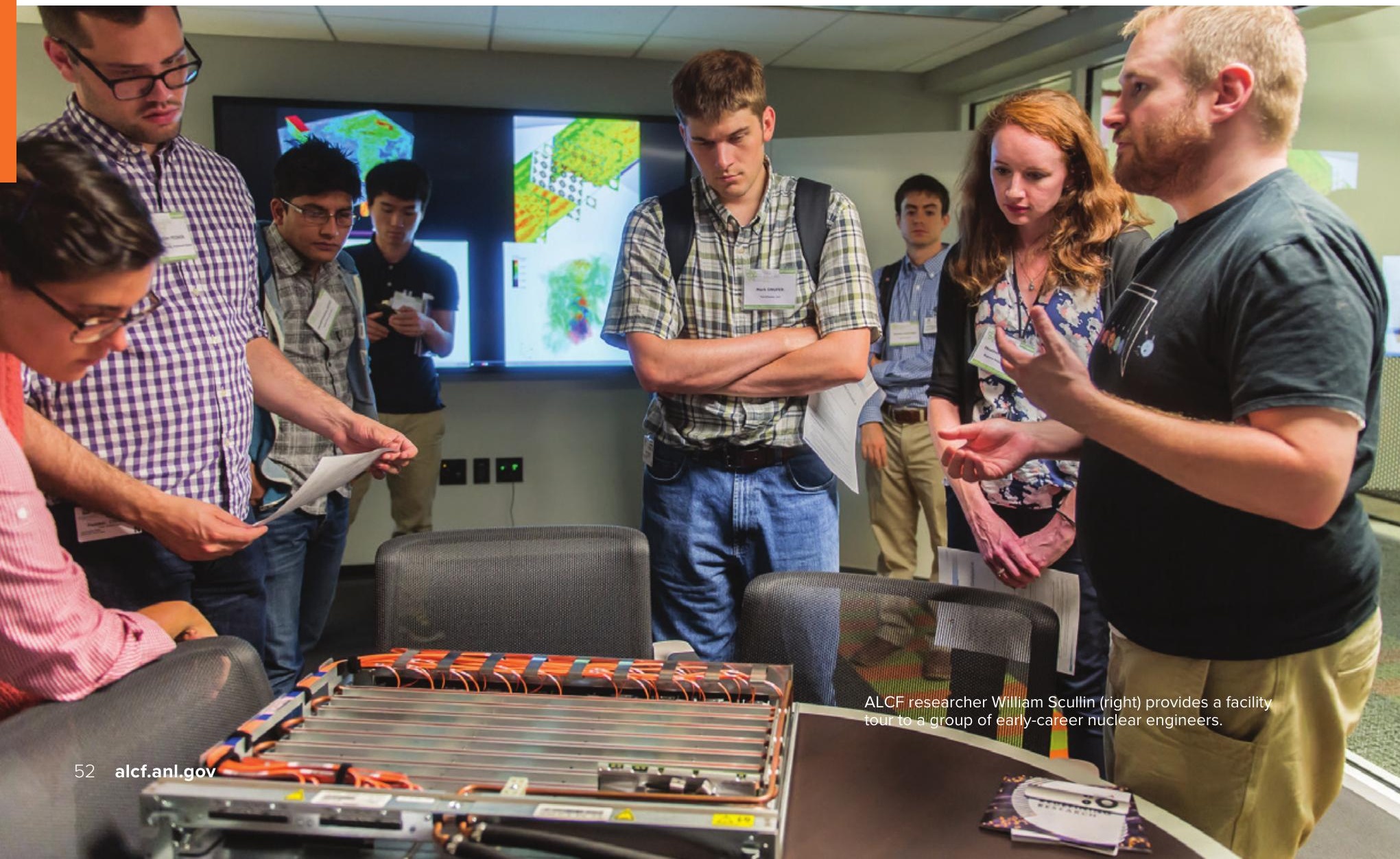




\section{GUIDING CURRENT AND FUTURE USERS}

\section{INCREASE Workshop}

This fall, Argonne partnered with the Interdisciplinary Consortium for Research and Education and Access in Science and Engineering (INCREASE) for a two-day workshop aimed at increasing the participation in and diversity of the user base at the laboratory's scientific user facilities, with a focus on engaging minorityserving institutions.

As part of the event, the ALCF held a brainstorming session to identify and better understand the barriers to gaining access to ASCR user facilities and how to overcome them. The partnership between INCREASE and Argonne has established a foundation for growing the next generation of STEM professionals and HPC users at minority-serving institutions.

\section{ATPESC}

For two weeks this summer, a group of 65 students and early career researchers attended the Argonne Training Program on Extreme-Scale Computing (ATPESC), a course designed to teach them the key skills and tools needed to efficiently use leading-edge supercomputers. Packed with technical lectures, handson exercises, and dinner talks, the arduous training program addresses all aspects of HPC with a curriculum that evolves each year to emphasize particular areas of interest.

This year, the organizers incorporated more hands-on sessions and placed increased focus on the importance of performance portability across diverse computing architectures.
The content was organized around seven core program tracks: hardware architectures; programming models and languages; numerical algorithms and FASTMath; community codes and software engineering; visualization and data analysis; toolkits and frameworks; and data-intensive computing and $\mathrm{I} / \mathrm{O}$. As with previous years, the ALCF recorded all of the 2015 ATPESC presentations and posted them to YouTube to extend the reach of the program.

\section{Mira Performance Boot Camp}

Now in its seventh year, the ALCF's Boot Camp event is a cornerstone of the facility's user training program. The three-day on-site workshop provides a timely opportunity for the user community to tap into the expertise of assembled ALCF staff and invited guests for help ramping up their code's scalability as they prepare to submit a proposal for an INCITE award.

The bulk of this year's event was devoted to hands-on, one-on-one tuning of applications. In addition, ALCF experts spoke on topics of interest, including Blue Gene/Q architecture, ensemble jobs, parallel $1 / O$, and data analysis. Guest speakers from tool and debugger vendors provided information and individualized assistance to attendees.

Reservation queues created specifically for the event gave the participants quick, uninterrupted access to ALCF resources, allowing them to run 835 jobs and to use more than 18.8 million core-hours as they diagnosed code issues and tweaked performance. This year, several groups were able to complete fullmachine runs on Mira and generate plots to incorporate into their INCITE proposals.

\section{Virtual Training for Users}

Employing an interactive videoconference format, the ALCF is able to connect with users from around the globe to provide virtual training on ALCF services and resources.

Offered several times a year, the Getting Started on ALCF Resources videoconference is targeted at new users and those in need of a refresher. The highly interactive sessions cover the basics that researchers need to get their projects up and running, and give users an opportunity to receive guided assistance in building their codes and submitting jobs on Mira.

New in 2015, the Ensemble Jobs for Better Throughput videoconference was developed for users whose workloads include multiple small jobs (<8K nodes) that are suitable to run concurrently. The specialized training session gave participants hands-on experience setting up an ensemble job script and helped them identify which job submission type is best for their projects.

The ALCF also used the virtual format for the Theta Early Science Program, holding videoconferences with project teams for the kickoff workshop and the program's first training session. 


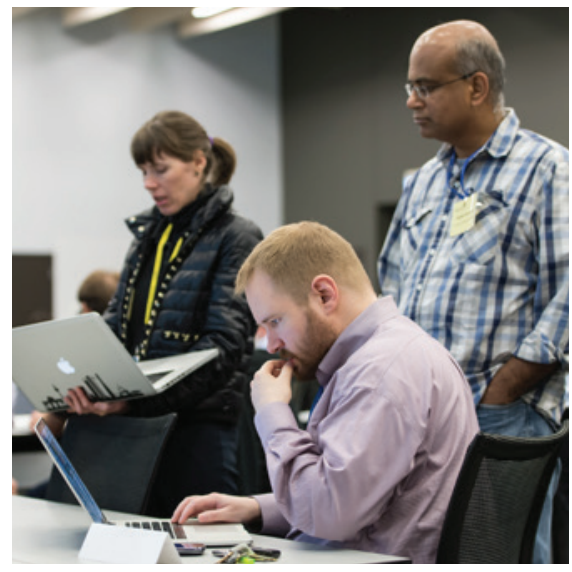

From introducing students to exciting career possibilities in HPC to working with users to inform them of new approaches for leadership-class systems, education is a critical part of the ALCF's mission.
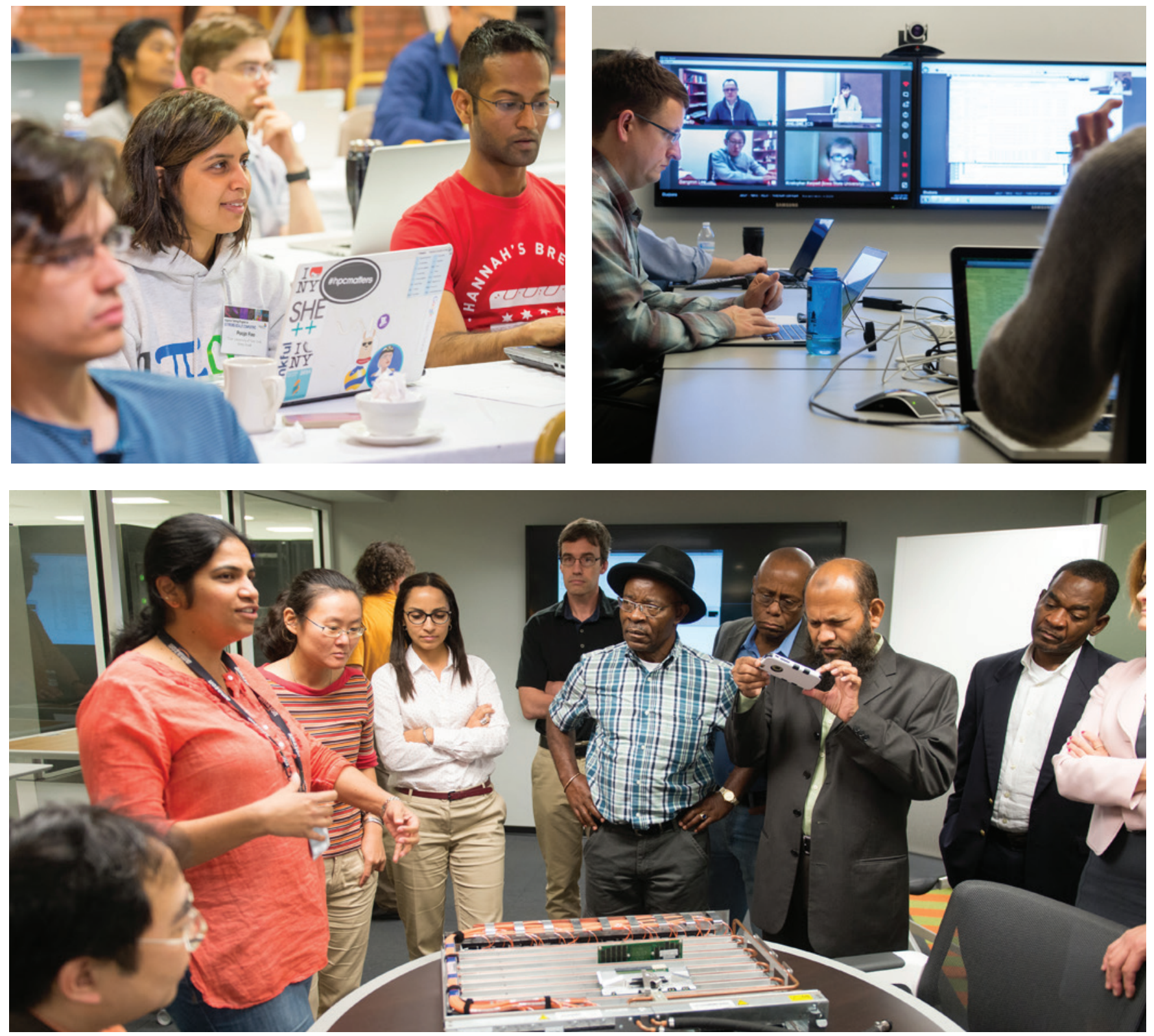

ALCF held or participated in a number of training and outreach events in 2015, including the Mira Performance Boot Camp (top), ATPESC (middle left), Getting Started videoconferences (middle right), and the INCREASE workshop (bottom). 
APPENDICES 


\section{ALCF PUBLICATIONS}

Researchers who use ALCF resources are major contributors to numerous publications that document their breakthrough science and engineering. The refereed journal articles and conference proceedings represent research ventures undertaken at the ALCF through programs supported by the U.S. Department of Energy and Argonne National Laboratory.

The publications are listed in descending order of their publication dates. An asterisk after a name indicates an Argonne author. ALCF publications are listed online at http://www.alcf.anl.gov/publications.

Barrett, B. R., E. Dikmen, A. F. Lisetskiy, P. Maris, A. M. Shirokov, and J. P. Vary. "Deriving the Nuclear Shell Model from First Principles." Journal of Physics: Conference Series, December 2015, vol. 580.

Bellwied, R., S. Borsányi, Z. Fodor, J. Günther, S. D. Katz, C. Ratti, and K. K. Szabó. "The QCD Phase Diagram from Analytic Continuation.” Physics Letters B, December 2015, vol. 751, 559-564.

Bellwied, R., S. Borsányi, Z. Fodor, S.D. Katz, A. Pásztor, C. Ratti, and K.K. Szabó. "Fluctuations and Correlations in High Temperature QCD.” Physical Review D, December 2015, vol. 92, no. 114505 .

Changlani, H. J., H. Zheng, and L. K. Wagner. "Density-Matrix Based Determination of Low-Energy Model Hamiltonians from Ab Initio Wavefunctions." Journal of Chemical Physics, December 2015, vol. 143, 102814.

Chen, C., ${ }^{*}$ Y.-L. S. Tse, G. E. Lindberg, C. Knight,* and G. A. Voth.* "Hydroxide Solvation and Transport in Anion Exchange Membranes." Journal of the American Chemical Society, December 2015, 991-1000.

Clough, K., P. Figueras, H. Finkel, ${ }^{*}$ M. Kunesch, E. A. Lim, and S. Tunyasuvunakool. "GRChombo: Numerical Relativity with Adaptive Mesh Refinement." Classical and Quantum Gravity, December 2015, vol. 32, no. 24.

Fang, A., H. Fujita, and A. A. Chien.* "Towards Understanding Post-Recovery Efficiency for Shrinking and Non-Shrinking Recovery." Euro-Par 2015: Parallel Processing Workshops, December 2015, vol. 9523, 656-668.

Hunold, S., A. Costan, D. Giménez, A. losup, L. Ricci, M. E. Gómez Requena, V. Scarano, A. L. Varbanescu, S. L. Scott, S. Lankes, J. Weidendorfer, and M. Alexander. Euro-Par 2015: Parallel Processing Workshops, Lecture Notes in Computer Science, December 2015, vol. 9523.
Li, X., E. Du, H. Lei, Y.-H. Tang, M. Dao, S. Suresh, and G. E. Karniadakis. "Patient-Specific Blood Rheology in Sickle-Cell Anaemia." Interface Focus, December 2015.

Li, Z., X. Bian, X. Li, and G. E. Karniadakis. "Incorporation of Memory Effects in Coarse-Grained Modeling via the Mori-Zwanzig Formalism." Journal of Chemical Physics, December 2015, vol. 143, 243128.

Li, Z., J. R. Kermode, and A. De Vita. "Molecular Dynamics with On-the-Fly Machine Learning of Quantum-Mechanical Forces." Physical Review Letters, December 2015, vol. 114, no. 9.

Li, Z., A. Yazdani, A. Tartakovsky, and G. E. Karniadakis. "Transport Dissipative Particle Dynamics Model for Mesoscopic Advection-Diffusion-Reaction Problems." Journal of Chemical Physics, December 2015, vol. 143, no. 1.

Liu, N., A. Haider, X.-H. Sun,, and D. Jin. "FatTreeSim: Modeling Large-Scale Fat-Tree Networks for HPC Systems and Data Centers Using Parallel and Discrete Event Simulation." Proceedings of the 3rd ACM SIGSIM Conference on Principles of Advanced Discrete Simulation, December 2015, London, England, 199-210.

Mahesh, K., P. Kumar,* A. Gnanaskandan, and Z. Nitzkorski. "LES Applied to Ship Research." Journal of Ship Science, December 2015, vol. 59, no. 4, 238-245.

Mosby, M. and K. Matouš. "Computational Homogenization at Extreme Scales." Extreme Mechanics Letters, December 2015, vol. 6, 68-74.

Olsen, K. and R. Takedatsu. "The SDSU Broadband Ground-Motion Generation Module BBtoolbox Version 1.5." Seismological Research Letters, December 2015, vol. 86, no. 1, 81-88.

Peguiron, A., L. C. Ciacchi, A. De Vita, J. R. Kermode, and G. Moras. "Accuracy of Buffered-Force QM/MM Simulations of Silica." Journal of Chemical Physics, December 2015, vol. 142, 064116. 
Ramakrishnan, R., M. Hartmann, E. Tapavicza, and O. A. von Lilienfeld.. "Electronic Spectra from TDDFT and Machine Learning in Chemical Space." Journal of Chemical Physics, December 2015, vol. 143, 084111.

Ratcliff, L. E.," L. Genovese, S. Mohr, and T. Deutsch. "Fragment Approach to Constrained Density Functional Theory Calculations Using Daubechies Wavelets." Journal of Chemical Physics, December 2015, vol. 142, no. 23.

Rosenberg, D., A. Pouquet, R. Marino, and P. D. Mininni. "Evidence for Bolgiano-Obukhov Scaling in Rotating Stratified Turbulence Using High-Resolution Direct Numerical Simulations." Physics of Fluids, December 2015, vol. 27.

Schiller, J. A., L. K. Wagner, and E. Ertekin. "Phase Stability and Properties of Manganese Oxide Polymorphs:

Assessment and Insights from Diffusion Monte Carlo." Physical Review B, December 2015, vol. 92, no. 23.

Sukys, J., U. Rasthofer, S. Verma, P. Hadjidoukas, D. Rossinelli, F. Wermelinger, B. Hejazialhosseini, and P. Koumoutsakos. "Numerical Investigation of Collapsing Cavity Arrays." Physics of Fluids, December 2015, vol. 24.

Withers, K. B., K. B. Olsen, and S. M. Day. "Memory-Efficient Simulation of Frequency-Dependent Q." Bulletin of the Seismological Society of America, December 2015, vol. 105, no. 6.

Yu, J., L. K. Wagner, and E. Ertekin. "Towards a Systematic Assessment of Errors in Diffusion Monte Carlo Calculations of Semiconductors: Case Study of Zinc Selenide and Zinc Oxide." Journal of Chemical Physics, December 2015, vol. 143, 224707.

Bai, Z., T. Blum, P.A. Boyle, N. H. Christ, J. Frison, N. Garron, T. Izubuchi, C. Jung, C. Kelly, C. Lehner, R.D. Mawhinney, C. T. Sachrajda, A. Soni, and D. Zhang. "Standard Model Prediction for Direct CP Violation in $\mathrm{K} \rightarrow \Pi \pi$ Decay." Physical Review Letters, November 2015, vol. 115, no. 21.

Dawson, W. and F. Gygi. "Performance and Accuracy of Recursive Subspace Bisection for Hybrid DFT Calculations in Inhomogeneous Systems." Journal of Chemical Theory and Computation, November 2015, vol. 11, no. 10, 4655-4663.

Feng, J. and I. Bolotnov. "Bubble-Induced Turbulence Study in Homogeneous Turbulent Flow Using DNS Approach." 68th Annual Meeting of the APS Division of Fluid Dynamics, November 2015, vol. 60, no. 21, Boston, Massachusetts.

Hu, L., K. D. Hammond, B. D. Wirth, and D. Maroudas. "Molecular-Dynamics Analysis of Mobile Helium Cluster Reactions Near Surfaces of Plasma-Exposed Tungsten." Journal of Applied Physics, November 2015, 163301.
Insley, J. A., J. Kodavasal, ${ }^{*}$ X. Chai, K. Harms,, M. García,* and S. Som.* "Gasoline Compression Ignition: Optimizing Start of Injection Time." SC15: The International Conference for High Performance Computing, Networking, Storage and Analysis, November 2015, Austin, Texas.

Keceli,* M., H. Zhang, ${ }^{*}$ P. Zapol, ${ }^{*}$ D. A. Dixon, and A. F. Wagner. "Shift-and-Invert Parallel Spectral Transformation Eigensolver: Massively Parallel Performance for Density-Functional Based Tight-Binding." Journal of Computational Chemistry, November 2015, vol. 37, no. 4, 448-459.

Kodavasal, J., ${ }^{*}$ K. Harms*, P. Srivastava, S. Som,* S. Quan, K. Richards, and M. García.* "Development of a StiffnessBased Chemistry Load Balancing Scheme, and Optimization of $\mathrm{I} / \mathrm{O}$ and Communication, to Enable Massively Parallel High-Fidelity Internal Combustion Engine Simulations." Proceedings of the American Society of Mechanical Engineers, November 2015, Houston, Texas.

Li, H., and J. L. Bredas. "Comparison of the Impact of Zinc Vacancies on Charge Separation and Charge Transfer at ZnO/Sexithienyl and ZnO/Fullerene Interfaces." Advanced Materials, November 2015.

Lu, C., X. Li, D. Wu, L. Zheng, and W. Yang. "Predictive Sampling of Rare Conformational Events in Aqueous Solution: Designing a Generalized Orthogonal Space Tempering Method." Journal of Chemical Theory and Computation, November 2015, vol. 12, no. 1, 41-52.

Malakar, P., ${ }^{*}$ V. Vishwanath,* T. Munson,* C. Knight,* M. Hereld,* S. Leyffer,* and M. E. Papka.* "Optimal Scheduling of In-Situ Analysis for Large-Scale Scientific Simulations."

SC "15: Proceedings of the International Conference for High Performance Computing, Networking, Storage and Analysis, November 2015, Austin, Texas.

Peng, I. B., S. Markidis, E. Laure, D. Holmes, and M. Bull. "A Data Streaming Model in MPI." Proceedings of the 3rd Workshop on Exascale MPI, November 2015, vol. 2, Austin, Texas.

Romero, N.A., ${ }^{*}$ A. Nakano, K. M. Riley,, F. Shimojo, R. K. Kalia, P. Vashishta, and P. C. Messina.* "Quantum Molecular Dynamics in the Post-Petaflops Era." Computer, November 2015, vol. 48, no. 11, 33-41.

Schlipf, M. and F. Gygi. "Optimization Algorithm for the Generation of ONCV Pseudopotentials." Computer Physics Communications, November 2015, vol. 196, 36-44. 
Shan, H., K. McElvain, C. Johnson, S. Williams, and W. E. Ormand. "Parallel Implementation and Performance Optimization of the Configuration-Interaction Method." SC '15 Proceedings of the International Conference for High Performance Computing, Networking, Storage and Analysis, November 2015, no. 9.

Shulenburger, L., A. D. Baczewski, Z. Zhu, J. Guan, and D. Tománek. "The Nature of the Interlayer Interaction in Bulk and Few-Layer Phosphorus." NANO Letters, November 2015, vol. 15, no. 12, 8170-8175.

Bailey, J. A., A. Bazavov, C. Bernard, C. M. Bouchard, C. DeTar, D. Du, A.X. El-Khadra, E.D. Freeland, E. Gámiz, S. Gottlieb, U.M. Heller, A.S. Kronfeld, J. Laiho, L. Levkova, Y. Liu, E. Lunghi, P.B. Mackenzie, Y. Meurice, E. Neil, S.-W. Qiu, J. N. Simone, R. Sugar, D. Toussaint, R. S. Van de Water, and R. Zhou. " $\mathrm{B} \rightarrow$ Ill Form Factors for New Physics Searches from Lattice QCD.” Physical Review Letters, October 2015, vol. 115, no. 15.

Blood-Forsythe, M. A., T. Markovich, R. A. DiStasio, Jr., R. Car, and A. Aspuru-Guzik. "Analytical Nuclear Gradients for the Range-Separated Many-Body Dispersion Model of Noncovalent Interactions." Chemical Science, October 2015.

Canepa, P., S. Jayaraman, L. Cheng,* N. N. Rajput,* W. D. Richards, G. S. Gautam, L. A. Curtiss, ${ }^{*}$ K. A. Perssond, and G. Ceder. "Elucidating the Structure of the Magnesium Aluminum Chloride Complex Electrolyte for Magnesium-Ion Batteries." Energy \& Environmental Science, October 2015, vol. 8, 3718-3730.

Gandolfi, S., A. Gezerlis, and J. Carlson. "Neutron Matter from Low to High Density." Annual Review of Nuclear and Particle Science, October 2015, vol. 65, 303-328.

Hammond, K. D. and B. D. Wirth. Erratum: "Crystal Orientation Effects on Helium Ion Depth Distributions and Adatom Formation Processes in Plasma-Facing Tungsten." Journal of Applied Physics, October 2015, vol. 118, 229901.

Narayanan,* B., S. A. Deshmukh,* S. K. R .S. Sankaranarayanan,* and S. Ramanathan. "Strong Correlations between Structural Order and Passive State at Water-Copper Oxide Interfaces." Electrochimica Acta, October 2015, vol. 179, 386-393.

Rizzi, S., ${ }^{*}$ M. Hereld, ${ }^{*}$ J. Insley,* M. E. Papka,, T. Uram,* and V. Vishwanath.* "Large-Scale Parallel Visualization of ParticleBased Simulations Using Point Sprites and Level-of-Detail." Proceedings of the 15th Eurographics Symposium on Parallel Graphics and Visualization (EGPGV), October 2015, Cagliari, Sardinia, Italy, 141-142.

Sewell, C., L. Lo, K. Heitmann,* S. Habib,* and J. Ahrens. "Utilizing Many-Core Accelerators for Halo and Center Finding within a Cosmology Simulation." 5th IEEE Symposium on Large Data Analysis and Visualization, October 2015, Chicago, Illinois, 91-98.
Wagner, L. K. "Ground State of Doped Cuprates from First-Principles Quantum Monte Carlo Calculations." Physical Review B, October 2015, vol. 92, no. 16.

Yan, L., J. E. Elenewski, W. Jiang,* and H. Chen. "Computational Modeling of Self-Trapped Electrons in Rutile $\mathrm{TiO}_{2}$." Physical Chemistry Chemical Physics, October 2015, vol. 17, 29949-29957.

Yu, H., J. Xie, K.-L. Ma, H. Kolla, and J. H. Chen. "Scalable Parallel Distance Field Construction for Large-Scale Applications." IEEE Transactions on Visualization and Computer Graphics, October 2015, vol. 21, no. 10, 1187-1200.

Bui, H., ${ }^{*}$ R. Jacob,* P. Malakar,* V. Vishwanath,* A. Johnson, M. E. Papka, ${ }^{*}$ and J. Leigh. "Multipath Load Balancing for $\mathrm{M} \times \mathrm{N}$ Communication Patterns on the Blue Gene/Q Supercomputer Interconnection Network." 2015 IEEE International Conference on Cluster Computing (CLUSTER), September 2015, Chicago, Illinois, 833-840.

Carlson, J., S. Gandolfi, F. Pederiva, S. C. Pieper,* R. Schiavilla, K.E. Schmidt, and R. B. Wiringa.* "Quantum Monte Carlo Methods for Nuclear Physics." Reviews of Modern Physics, September 2015, vol. 87, no. 3.

Fodor, Z., K. Holland, J. Kuti, S. Mondal, D. Nogradi, and C. H. Wong. "The Running Coupling of the Minimal Sextet Composite Higgs Model.” Journal of High Energy Physics, September 2015.

Isaila, F., P. Balaprakash,* S. M. Wild,, D. Kimpe, R. Latham,* R. Ross, ${ }^{*}$ and P. Hovland.* "Collective I/O Tuning Using Analytical and Machine Learning Models." 2015 IEEE International Conference on Cluster Computing (CLUSTER), September 2015, Chicago, Illinois, 128-137.

Kermode, J. R., A. Gleizer, G. Kovel, L. Pastewka, G. Csányi, D. Sherman, and A. De Vita. "Low Speed Crack Propagation via Kink Formation and Advance on the Silicon (110) Cleavage Plane." Physical Review Letters, September 2015, vol. 115, no. 13-25.

Klawonn, A., M. Lanser, and O. Rheinbach. "Toward Extremely Scalable Nonlinear Domain Decomposition Methods for Elliptic Partial Differential Equations." Software and High Performance Computing, September 2015, vol. 37, no. 6, 667-696.

Li, X., C. Lv, K. M. Corbett, L. Zheng, D. Wu, and W. Yang. "Free Energy Landscape of a Minimalist Salt Bridge Model." Protein Science, September 2015, 270-276.

Malakar, P.* and V. Vishwanath.* "Hierarchical Read-Write Optimizations for Scientific Applications with Multi-Variable Structured Datasets." Proceedings of the 12th Annual IFIP International Conference on Network and Parallel Computing (NPC), September 2015, New York, New York. 
Pruitt, S. R., ${ }^{*}$ K. R. Brorsen, and M. S. Gordon. "Ab Initio Investigation of the Aqueous Solvation of the Nitrate Ion." Physical Chemistry Chemical Physics, September 2015, vol. 27, 27027-27034.

Sewell, C., K. Heitmann, ${ }^{*}$ H. Finkel,, G. Zagaris, S. T. Parete-Koon, P. Fasel, A. Pope, ${ }^{*}$ N. Frontiere, ${ }^{*}$ L. Lo, B. Messer, S. Habib,* and J. Ahrens. "Large-Scale Compute-Intensive Analysis via a Combined In-Situ and Co-Scheduling Workflow Approach." SC '15 Proceedings of the International Conference for High Performance Computing, Networking, Storage and Analysis, September 2015, Austin, Texas.

Tang, Y.-H., S. Kudo, X. Bian, Z. Li, and G. E. Karniadakis. "Multiscale Universal Interface: A Concurrent Framework for Coupling Heterogeneous Solvers." Journal of Computational Physics, September 2015, vol. 297, 13-31.

Wallace, S., V. Vishwanath," S. Coghlan,* Z. Lan, and M. E. Papka.* "Comparison of Vendor Supplied Environmental Data Collection Mechanisms." 2015 IEEE International Conference on Cluster Computing (CLUSTER), September 2015, Chicago, Illinois, 690-697.

Weichselbaum, N. A., M. R. Abkenar, M. Vanella, M. T. Manzari, E. Balaras, and P. M. Bardet. "Validation Facility and Model Development for Nuclear Fuel Assembly Response to Seismic Loading." Journal of Nuclear Engineering and Radiation Science, September 2015, vol. 1, no. 4.

Yazdani, A., X. Li, and G. E. Karniadakis. "Dynamic and Rheological Properties of Soft Biological Cell Suspensions." Rheologica Acta, September 2015, 1-17.

Bailey, J. A., A. Bazavov, C. Bernard, C. M. Bouchard, C. DeTar, D. Du, A.X. El-Khadra, J. Foley, E. D. Freeland, E. Gámiz, S. Gottlieb, U. M. Heller, J. Komijani, A. S. Kronfeld, J. Laiho, L. Levkova, P. B. Mackenzie, E. T. Neil, S.-W. Qiu, J. Simone, R. Sugar, D. Toussaint, R. S. Van de Water, and R. Zhou. "B $\rightarrow$ DPv Form Factors at Nonzero Recoil and IVcbl from 2+1-Flavor Lattice QCD." Physical Review D, August 2015, vol. 92, no. 3.

Bankura, A., B. Santra, R. A. DiStasio Jr., C. W. Swartz, M. L. Klein, and X. Wu. "A Systematic Study of Chloride Ion Solvation in Water Using van der Waals Inclusive Hybrid Density Functional Theory." Molecular Physics, July 2015, vol. 113, no. 17-18.

Doolaard, H. J., A. Shams, F. Roelofs, K. Van Tichelen, S. Keijers, J. De Ridder, J. Degroote, J. Vierendeels, I. Di Piazza, E. Merzari," A. Obabko, ${ }^{*}$ and P. Fischer.* "CFD Benchmark for a Heavy Liquid Metal Fuel Assembly." Proceedings of NURETH-16, August 2015, Chicago, Illinois.
Heitmann, K., ${ }^{*}$ N. Frontiere, ${ }^{*}$ C. Sewell, S. Habib, ${ }^{*}$ A. Pope, ${ }^{*}$ H. Finkel, ${ }^{*}$ S. Rizzi, ${ }^{*}$ J. Insley, ${ }^{*}$ and S. Bhattacharya.* "The Q Continuum Simulation: Harnessing the Power of GPU Accelerated Supercomputers." Astrophysical Journal, August 2015, vol. 219, no. 2.

Lozosa, J. C., K. B. Olsen, J. N. Brune, R. Takedatsu, R. J. Brune, and D. D. Oglesby. "Broadband Ground Motions from Dynamic Models of Rupture on the Northern San Jacinto Fault, and Comparison with Precariously Balanced Rocks." Bulletin of the Seismological Society of America, August 2015, vol. 105, no. 4, 1947-1960.

Lykov, K., X. Li, H. Lei, I. V. Pivkin, and G. E. Karniadakis. "Inflow/Outflow Boundary Conditions for Particle-Based Blood Flow Simulations: Application to Arterial Bifurcations and Trees." Computational Biology, August 2015.

Maheshwari, K., J. M. Wozniak, ${ }^{*}$ T. G. Armstrong, D. S. Katz,* T. A. Binkowski, X. Zhong, ${ }^{*}$ O. Heinonen, ${ }^{*}$ D. Karpeyev, and M. Wilde.* "Porting Ordinary Applications to Blue Gene/Q Supercomputers." 2015 IEEE 11th International Conference on eScience. August 2015, Munich, Germany, 420-428.

Nomura, K., P. E. Small, R. K. Kalia, A. Nakano, and P. Vashishta. "An Extended-Lagrangian Scheme for Charge Equilibration in Reactive Molecular Dynamics Simulations." Computer Physics Communications, July 2015, vol. 192, 91-96.

Prabhat, S. B., V. Vishwanath,, E. Dart, M. Wehner, and W. D. Collins. "TECA: Petascale Pattern Recognition for Climate Science." Computer Analysis of Images and Patterns, August 2015, vol. 8257, 426-436.

Radice, D., S. M. Couch, and D. Ott. "Implicit Large Eddy Simulations of Anisotropic Weakly Compressible Turbulence with Application to Core-Collapse Supernovae." Computational Astrophysics and Cosmology, August 2015.

Ren, X., N. Marom, F. Caruso, M. Scheffler, and P. Rinke. "Beyond the GW Approximation: A Second-Order Screened Exchange Correction." Physical Review B, August 2015, vol. 92, no. 8.

Santra, B., R. A. DiStasio Jr., F. Martelli, and R. Car. "Local Structure Analysis in Ab Initio Liquid Water." Molecular Physics, July 2015, vol. 113, no. 17-18.

Wirth, B. D., K. D. Hammond, S. I. Krasheninnikov, and D. Maroudas. "Challenges and Opportunities of Modeling Plasma-Surface Interactions in Tungsten Using HighPerformance Computing." Journal of Nuclear Materials, August 2015, vol. 463, 30-38. 
Zarzycki, C. M., K. A. Reed, J. Bacmeister, A. P. Craig, S. C. Bates, and N. A. Rosenbloom. "Impact of Ocean Coupling Strategy on Extremes in High-Resolution Atmospheric Simulations." Geoscientific Model Development, August 2015, vol. 8, no. 7983-8007.

Alsamarah, A. E., P. LaCuran, J. Oelschlaeger, J. Hao, and Y. Luo. "Uncovering Molecular Bases Underlying Bone Morphogenetic Protein Receptor Inhibitor Selectivity." PLOS One, July 2015.

Bailey, J. A., A. Bazavov, C. Bernard, C. M. Bouchard, C. DeTar, D. Du, A. X. El-Khadra, J. Foley, E. D. Freeland, E. Gámiz, S. Gottlieb, U. M. Heller, J. Komijani, A. S. Kronfeld, J. Laiho, L. Levkova, Y. Liu, P. B. Mackenzie, Y. Meurice, E. T. Neil, S.-W. Qiu, J. Simone, R. Sugar, D. Toussaint, R. S. Van de Water, and R. Zhou. "IVubl from $B \rightarrow$ $\rightarrow$ Pv Decays and (2+1)-Flavor Lattice QCD." Physical Review D, July 2015, vol. 92, no. 1.

Couch, S. M., E. Chatzopoulos, W. D. Arnett, and F. X. Timmes. "The Three-Dimensional Evolution to Core Collapse of a Massive Star." Astrophysical Journal Letters, July 2015, vol. 808, no. 1.

Macridin, A., A. Burov, E. Stern, J. Amundson, and P. Spentzouris. "Simulation of Transverse Modes with Their Intrinsic Landau Damping for Bunched Beams in the Presence of Space Charge." Physical Review Accelerators and Beams, July 2015, vol. 18, no. 7.

Oryspayev, D., H. M. Aktulga, M. Sosonkina, P. Maris, and J .P. Vary. "Performance Analysis of Distributed Symmetric Sparse Matrix Vector Multiplication Algorithm for Multi-Core Architectures." Concurrency and Computation: Practice and Experience, July 2015, vol. 27, no. 17, 5019-5036.

Perdikaris, P., D. Venturi, J. O. Royset, and G. E. Karniadakis. "Multi-Fidelity Modelling via Recursive Co-Kriging and Gaussian-Markov Random Fields." Proceedings of the Royal Society A, July 2015.

Wang, J.* and V. R. Kotamarthi.* "High-Resolution Dynamically Downscaled Projections of Precipitation in the Mid and Late 21st Century over North America." Earth's Future, July 2015, vol. 3, no. 7, 268-288.

Boyle, P. A., N. H. Christ, J. M. Flynn, N. Garron, C. Jung, A. Jüttner, R. D. Mawhinney, D. Murphy, C. T. Sachrajda, F. Sanfilippo, and H. Yin. "The Kaon Semileptonic Form Factor in N f = 2 + 1 Domain Wall Lattice QCD with Physical Light Quark Masses." Theoretical Physics, June 2015.

Caccin, M., Z. Li, J. R. Kermode, and A. De Vita. "A Framework for Machine-Learning-Augmented Multiscale Atomistic Simulations on Parallel Supercomputers." International Journal of Quantum Chemistry, June 2015.
Chien, A., ${ }^{*}$ P. Balaji, ${ }^{*}$ P. Beckman,, N. Dun,* A. Fang, H. Fujita, K. Iskra, ${ }^{*}$ Z. Rubenstein, Z. Zheng, ${ }^{*}$ R. Schreiber, J. Hammond, J. Dinan, I. Laguna, D. Richards, A. Dubey, B. van Straalenf, M. Hoemmen, M. Heroux, K. Teranishi, and A. Siegel.* "Versioned Distributed Arrays for Resilience in Scientific Applications: Global View Resilience." Procedia Computer Science, June 2015, vol. 51, 29-38.

Colquhoun, B., C. T. H. Davies, J. Kettle, J. Koponen, A. T. Lytle, R. J. Dowdall, and G.P. Lepage. "B-Meson Decay Constants: A More Complete Picture from Full Lattice QCD." Physical Review D, June 2015, vol. 91, no. 11.

Dikmen, E., A. F. Lisetskiy, B. R. Barrett, P. Maris, A. M. Shirokov, and J. P. Vary. "Ab Initio Effective Interactions for Sd-Shell Valence Nucleons." Physical Review Letters, June 2015.

Fodor, Z., K. Holland, J. Kuti, S. Mondal, D. Nogradi, and C. H. Won. "The Running Coupling of 8 Flavors and 3 Colors." Journal of High Energy Physics, June 2015.

Gaiduk, A. P., F. Gygi, and G. Galli.* "Density and Compressibility of Liquid Water and Ice from First-Principles Simulations with Hybrid Functionals." Journal of Physical Chemistry Letters, June 2015, vol. 6, no. 15, 2902-2908.

Ghosh, D., E. M. Constantinescu,* and J. Brown. "Efficient Implementation of Nonlinear Compact Schemes on Massively Parallel Platforms." SIAM Journal on Scientific Computing, June 2015, vol. 37, no. 3, 354-383.

Hammond, K. D., L. Hu, D. Maroudas, and B. D. Wirth. "Helium Impurity Transport on Grain Boundaries: Enhanced or Inhibited?" Europhysics Letters, June 2015, vol. 110, no. 5.

Hansen, K., F. Biegler, R. Ramakrishnan, W. Pronobis, O. A. von Lilienfeld, ${ }^{*}$ K.-R. Müller, and A. Tkatchenko. "Machine Learning Predictions of Molecular Properties: Accurate Many-Body Potentials and Nonlocality in Chemical Space." Journal of Physical Chemistry Letters, June 2015, vol. 6, no. 12, 2326-2331.

Jiang, Z., J. He, S. A. Deshmukh, P. Kanjanaboos, G. Kamath, Y. Wang, S. K. R. S. Sankaranarayanan, J. Wang, H. M. Jaeger, and X.-M. Lin. "Subnanometre Ligand-Shell Asymmetry Leads to Janus-Like Nanoparticle Membranes. Nature Materials, June 2015, vol. 14, 912-917

Lovato, A.,* S. Gandolfi, J. Carlson, S. C. Pieper,* and R. Schiavilla. "Electromagnetic and Neutral-Weak Response Functions of He4 and C12." Physical Review C, June 2015, vol. 91, no. 6 .

Lv, C., E. W. Aitchison, D. Wu, L. Zheng, X. Cheng, and W. Yang. "Comparative Exploration of Hydrogen Sulfide and Water Transmembrane Free Energy Surfaces via Orthogonal Space Tempering Free Energy Sampling." Journal of Computational Chemistry, June 2015. 
Park, H., F. DiMaio, and D. Baker. "The Origin of Consistent Protein Structure Refinement from Structural Averaging." Structure, June 2015, 1123-1128.

Amer, A., ${ }^{*}$ H. Lu, P. Balaji,* and S. Matsuoka. "Characterizing MPI and Hybrid MPI+Threads Applications at Scale: Case Study with BFS." 15th IEEE/ACM International Symposium on Cluster, Cloud and Grid Computing, May 2015, Shenzhen, China, 1075-1083.

Bereau, T., D. Andrienko, and O. A. von Lilienfeld.* "Transferable Atomic Multipole Machine Learning Models for Small Organic Molecules." Journal of Chemical Theory and Computation, May 2015, vol. 11, no. 7, 3225-3233.

Berman, D., S. A. Deshmukh,* S. K. R .S. Sankaranarayanan,* A. Erdemir,* A. V. Sumant.* "Macroscale Superlubricity Enabled by Graphene Nanoscroll Formation." Science, May 2015, vol. 348, 1118.

Chen, H., F. Fiuza, A. Link, A. Hazi, M. Hill, D. Hoarty, S. James, S. Kerr, D.D. Meyerhofer, J. Myatt, J. Park, Y. Sentoku, and G. J. Williams. "Scaling the Yield of Laser-Driven ElectronPositron Jets to Laboratory Astrophysical Applications." Physical Review Letters, May 2015, vol. 114, no. 215001.

Deng, M., L. Grinberg, B. Caswell, and G. E. Karniadakis. "Effects of Thermal Noise on the Transitional Dynamics of an Inextensible Elastic Filament in Stagnation Flow." Soft Matter, May 2015, vol. 11, 4962-4972.

Ekström, A., G. R. Jansen, K. A. Wendt, G. Hagen, T. Papenbrock, B. D. Carlsson, C. Forssén, M. Hjorth-Jensen, P. Navrátil, and W. Nazarewicz. "Accurate Nuclear Raddi and Binding Energies from a Chiral Interaction." Physical Review C, May 2015, vol. 91.

Jain, N., A. Bhatele, J.-S. Yeom, M. F. Adams, F. Miniati, C. Mei, and L. V. Kale. "Charm++ and MPI: Combining the Best of Both Worlds." Department of Computer Science, University of Illinois at Urbana-Champaign, Urbana, Illinois, May 2015, 655-664.

Meng, J., ${ }^{*}$ V. Morozov,* T. Uram,, V. Vishwanath,* and K. Kumaran.* "Modeling Cooperative Threads to Project GPU Performance for Adaptive Parallelism." Proceedings of 16th International Workshop on Parallel and Distributed Scientific and Engineering Computing, May 2015, Hyderabad, India, 998-1007.

Meng, J., S. Seo,* P. Balaji,* and Y. Wei. "SWAP-Assembler 2: Scalable Genome Assembler towards Millions of CoresPractice and Experience." 15th IEEE/ACM International Symposium on Cluster, Cloud and Grid Computing, May 2015, Shenzhen, China, 769-772.
Reed, K. A., J. T. Bacmeister, N. A. Rosenbloom, M. F. Wehner, S. C. Bates, P. H. Lauritzen, J. E. Truesdale, and C. Hannay. "Impact of the Dynamical Core on the Direct Simulation of Tropical Cyclones in a High-Resolution Global Model." Geophysical Research Letters, May 2015, vol. 42, no. 9, 3603-3608.

Signoracci, A., T. Duguet, G. Hagen, and G. R. Jansen. "Ab Initio Bogoliubov Coupled Cluster Theory for Open-Shell Nuclei." Physical Review C, May 2015, vol. 91, no. 6.

Wu, J., X. Xiong, and Z. Lan. "Hierarchical Task Mapping for Parallel Applications on Supercomputers." Journal of Supercomputing, May 2015, vol. 71, no. 5, 1776-1802.

Blum, T., P.A. Boyle, N. H. Christ, J. Frison, N. Garron, T. Janowski, C. Jung, C. Kelly, C. Lehner, A. Lytle, R.D. Mawhinney, C. T. Sachrajda, A. Soni, H. Yin, and D. Zhang. " $\mathrm{K} \rightarrow \Pi \Pi \Delta \mathrm{I}=3 / 2$ Decay Amplitude in the Continuum Limit." Physical Review D, April 2015, vol. 91, no. 7.

Canepa, P., G. S. Gautam, R. Malik, S. Jayaraman, Z. Rong, K. R. Zavadil,* K. Persson, and G. Ceder. "Understanding the Initial Stages of Reversible Mg Deposition and Stripping in Inorganic Nonaqueous Electrolytes." Chemistry of Materials, April 2015, vol. 27, no. 9, 3317-3325.

Faber, F., A. Lindmaa, O. A. von Lilienfeld, ${ }^{*}$ and R. Armiento. "Crystal Structure Representations for Machine Learning Models of Formation Energies." International Journal of Quantum Chemistry, April 2015.

Ferri, N., R. A. DiStasio, Jr., A. Ambrosetti, R. Car, and A. Tkatchenko. "Electronic Properties of Molecules and Surfaces with a Self-Consistent Interatomic van der Waals Density Functional." Physical Review Letters, April 2015, vol. 114, no. 17.

Hinohara, N., M. Kortelainen, W. Nazarewicz, and E. Olsen. "Complex-Energy Approach to Sum Rules within Nuclear Density Functional Theory." Physical Review C, April 2015, vol. 91, no. 4.

Hura, S.-M., V. Thapar, A. Ramírez-Hernández, ${ }^{*}$ G. Khaira, T. Segal-Peretz,* P. A. Rincon-Delgadillo, W. Li, M. Müller, P. F. Nealey, and J. J. de Pablo. "Molecular Pathways for Defect Annihilation in Directed Self-Assembly." Proceedings of the National Academy of Sciences, April 2015, vol. 112, no. 46, 14144-14149.

von Lilienfeld, O. A., ${ }^{*}$ R. Ramakrishnan, M. Rupp, and A. Knoll. "Fourier Series of Atomic Radial Distribution Functions: A molecular Fingerprint for Machine Learning Models of Quantum Chemical Properties." International Journal of Quantum Chemistry, April 2015. 
Lo, Y., J., S. Williams, B. Van Straalen, T. J. Ligocki,

M. J. Cordery, N. J. Wright, M. W. Hall, and L. Oliker. "Roofline

Model Toolkit: A Practical Tool for Architectural and Program Analysis." High Performance Computing Systems: Performance Modeling, Benchmarking, and Simulation, April 2015, vol. 8966, 129-148.

Ramakrishnan, R., P. O. Dral, M. Rupp, and O. A. von Lilienfeld.* "Big Data Meets Quantum Chemistry Approximations: The $\triangle$-Machine Learning Approach." Journal of Chemical Theory and Computation, April 2015, vol. 11, no. 5, 2087-2096.

Ramakrishnan, R. and O. A. von Lilienfeld.* "Many Molecular Properties from One Kernel in Chemical Space." CHIMIA International Journal for Chemistry, April 2015, vol. 69, no. 4, 182-186.

Ratcliff, L. E., ${ }^{*}$ L. Grisanti, L. Genovese, T. Deutsch, T. Neumann, D. Danilov, W. Wenzel, D. Beljonne, and J. Cornil. "Toward Fast and Accurate Evaluation of Charge On-Site Energies and Transfer Integrals in Supramolecular Architectures Using Linear Constrained Density Functional Theory (CDFT)-Based Methods." Journal of Chemical Theory and Computation, April 2015, vol. 11, no. 5, 2077-2086.

Shaw, J. H., A. Plesch, C. Tape, M. P. Suess. T. H. Jordan, G. Ely, E. Hauksson. J. Tromp, T. Tanimoto, R. Graves, K. Olsen, C. Nicholson, P. J. Maechling, C. Rivero, P. Lovely, C. M. Brankman, and J. Munster. "Unified Structural Representation of the Southern California Crust and Upper Mantle." Earth and Planetary Science Letters, April 2015, vol. 415, no. 1, 1-15.

Ferroni, F., K. D. Hammond, and B. D. Wirth. "Sputtering Yields of Pure and Helium-Implanted Tungsten under FusionRelevant Conditions Calculated Using Molecular Dynamics." Journal of Nuclear Materials, March 2015, vol. 458, 419-424.

Jordan, T.H. "An Effective Medium Theory for ThreeDimensional Elastic Heterogeneities." Geophysical Journal International, March 2015, vol. 203, no. 2, 1343-1354.

Kim, C. and G. E. Karniadakis. "Brownian Motion of a Rayleigh Particle Confined in a Channel: A Generalized Langevin Equation Approach." Journal of Statistical Physics, March 2015, vol. 158, no. 5, 1100-1125.

Lonardoni, D., ${ }^{*}$ A. Lovato,* S. Gandolfi, and F. Pederiva. "Hyperon Puzzle: Hints from Quantum Monte Carlo Calculations." Physical Review Letters, March 2015, vol. 114, no. 9.

Maheshwari, K., ${ }^{*}$ E. Jung, ${ }^{*}$ J. Meng, ${ }^{*}$ V. Morozov, V. Vishwanath," and R. Kettimuthu." "Workflow Performance Improvement Using Model-Based Scheduling over Multiple Clusters and Clouds." Future Generation Computer Systems, March 2015, vol. 54, 206-218.
McDonnell, J. D., N. Schunck, D. Higdon, J. Sarich,* S. M. Wild," and W. Nazarewicz. "Uncertainty Quantification for Nuclear Density Functional Theory and Information Content of New Measurements." Physical Review Letters, March 2015, vol. 114, no. 12.

Schumacher, J., P. Götzfried, and J. D. Scheel. “Enhanced Enstrophy Generation for Turbulent Convection in LowPrandtl-Number Fluids." Proceedings of the National Academy of Sciences of the United States of America, March 2015, vol. 112, no. 31, 9530-9535.

Terasaki, J. "Many-Body Correlations of Quasiparticle Random-Phase Approximation in Elements of Neutrinoless Double- $\beta$ Decay." Physical Review C, March 2015, vol. 91, no. 3.

Ballaka, S. B., R. T. Jaspers, L. Deldicque, S. Chalil, E. L. Peters, A. de Haan, and H. Degens. "Blunted Hypertrophic Response in Old Mouse Muscle Is Associated with a Lower Satellite Cell Density and Is Not Alleviated by Resveratrol." Experimental Gerontology, February 2015, vol. 62, 23-31.

Bao, Y., R. L. Martin, M. Haranczyk, and M. W. Deem. "In Silico Prediction of MOFs with High Deliverable Capacity or Internal Surface Area." Physical Chemistry Chemical Physics, February 2015, vol. 17, 11962-11973.

Beresnyak, A. "On the Parallel Spectrum in Magnetohydrodynamic Turbulence." Astrophysical Journal Letters, February 2015, vol. 801, no. 1.

Bitzek, E., J. R. Kermode, and P. Gumbsch. "Atomistic Aspects of Fracture." International Journal of Fracture, February 2015, vol. 191, no. 1, 13-30.

Dytrych, T., A. C. Hayes, K. D. Launey, J. P. Draayer, P. Maris, J. P. Vary, D. Langr, and T. Oberhuber. "Electron-Scattering Form Factors for ${ }^{6} \mathrm{Li}$ in the Ab Initio Symmetry-Guided Framework." Physical Review C, February 2015, vol. 91, no. 2.

Ekström, A., B. D. Carlsson, K. A. Wendt, C. Forssén, M. Hjorth-Jensen, R. Machleidt, and S. M. Wild.* "Statistical Uncertainties of a Chiral Interaction at Next-to-Next-to Leading Order." Journal of Physics G: Nuclear and Particle Physics, February 2015, vol. 42, no. 3.

Furnstahl, R. J., G. Hagen, T. Papenbrock, and K. A. Wendt. "Infrared Extrapolations for Atomic Nuclei." Journal of Physics G: Nuclear and Particle Physics, February 2015.

Higdon, D., J. D. McDonnell, N. Schunck, J. Sarich,* and S. M. Wild.* "A Bayesian Approach for Parameter Estimation and Prediction Using a Computationally Intensive Model." Journal of Physics G: Nuclear and Particle Physics, February 2015, vol. 42, no. 3. 
Ireland, D. G. and W. Nazarewicz. "Enhancing the Interaction between Nuclear Experiment and Theory through Information and Statistics." Journal of Physics G: Nuclear and Particle Physics, February 2015, vol. 42, no. 3.

Isbiliroglu, Y., R. Taborda, and J. Bielak. "Coupled SoilStructure Interaction Effects of Building Clusters During Earthquakes." Earthquake Spectra, February 2015, vol. 31, no. 1, 463-500.

Langhammer, J., P. Navrátil, S. Quaglioni, G. Hupin, A. Calci, and R. Roth. "Continuum and Three-Nucleon Force Effects on 9Be Energy Levels." Physical Review C, February 2015, vol. 91, no. 2.

Li, Z., Y.-H. Tang, X. Lia, and G. E. Karniadakis. "Mesoscale Modeling of Phase transition Dynamics of Thermoresponsive Polymers." Chemical Communications, February 2015, vol. 51, 11038-11040.

Papadimitriou, G. and J. P. Vary. "Nucleon-Nucleon Resonances at Intermediate Energies Using a Complex Energy Formalism." Physics Letters B, February 2015, vol. 746, 121-126.

Papadimitriou, G. and J. P. Vary. "Nucleon-Nucleon Scattering with the Complex Scaling Method and Realistic Interactions." Physical Review C, February 2015, vol. 91, no. 2.

Schunck, N., J. D. McDonnell, J. Sarich,* S. M. Wild,* and D. Higdon. "Error Analysis in Nuclear Density Functional Theory." Journal of Physics G: Nuclear and Particle Physics, February 2015.

Wang, J., ${ }^{*}$ F. N. U. Swati, M. L. Stein, and V. R. Kotamarthi.* "Model Performance in Spatiotemporal Patterns of Precipitation: New Methods for Identifying Value Added by a Regional Climate Model." Journal of Geophysical Research Atmospheres, February 2015, vol. 10, no. 4, 1239-1259.

Yanai, T., G. I. Fann, G. Beylkinc, and R. J. Harrison. "Multiresolution Quantum Chemistry in Multiwavelet Bases: Excited States from Time-Dependent Hartree-Fock and Density Functional Theory via Linear Response." Physical Chemistry Chemical Physics, February 2015, vol. 17, 31405-31416.

Al-Hamdani, Y. S., M. Ma, D. Alfè, O. A. von Lilienfeld, ${ }^{*}$ and A. Michaelides. "Communication: Water on Hexagonal Boron Nitride from Diffusion Monte Carlo." Journal of Chemical Physics, January 2015, vol. 42, no. 181101.

Bai, P., M. Y. Jeon, L. Ren, C. Knight,* M. W. Deem, M. Tsapatsis, and J. I. Siepmann. "Discovery of Optimal Zeolites for Challenging Separatons and Chemical Transformations Using Predictive Materials Modeling." Nature Communications, January 2015, no. 5912.
Couch, S. M. and C. D. Ott. "The Role of Turbulence in Neutrino-Driven Core-Collapse Supernova Explosions.” Astrophysical Journal, January 2015, vol. 799, no. 1.

Gillan, M. J., D. Alfè, and F. R. Manby. "Energy Benchmarks for Methane-Water Systems from Quantum Monte Carlo and Second-Order Møller-Plesset Calculations." Journal of Chemical Physics, January 2015, vol. 143, no. 102812.

Govoni, M.* and G. Galli.* "Large Scale GW Calculations." Journal of Chemical Theory and Computation, January 2015.

Huntington, C. M., F. Fiuza, J. S. Ross, A. B. Zylstra, R. P. Drake, D. H. Froula, G. Gregori, N. L. Kugland, C. C. Kuranz, M. C. Levy, C. K. Li, J. Meinecke, T. Morita, R. Petrasso, C. Plechaty, B. A. Remington, D. D. Ryutov, Y. Sakawa, A. Spitkovsky, H. Takab, and H.-S. Park. "Observation of Magnetic Field Generation via the Weibel Instability in Interpenetrating Plasma Flows." Nature Physics, January 2015, vol. 11, 173-176.

Li, Z., X. Bian, X. Li, and G. E. Karniadakis. "Incorporation of Memory Effects in Coarse-Grained Modeling via the Mori-Zwanzig Formalism." Journal of Chemical Physics, January 2015, vol. 143.

Maris, P., M. A. Caprio, and J. P. Vary. "Emergence of Rotational Bands in Ab Initio No-Core Configuration Interaction Calculations of the Be Isotopes." Physical Review C, January 2015, vol. 91, no. 1.

Nashed, Y. S. G., ${ }^{*}$ D. J. Vine,* T. Peterka, J. Deng, ${ }^{*}$ R. Ross,* and C. Jacobsen.* "Parallel Ptychographic Reconstruction." Optics InfoBase-Optics Express, January 2015, vol. 22, no. 26, 32082-32097.

Sack, P. and W. Gropp. "Collective Algorithms for Multiported Torus Networks." ACM Transactions on Parallel Computing: Special Issue on PPOPP 2012, January 2015, vol. 1, no. 2.

Sanchez, E., C. Paolini, P. Blomgren, and J. Castillo. "Algorithms for Higher-Order Mimetic Operators." Spectral and High Order Methods for Partial Differential Equations, January 2015, vol. 106, 424-434.

Steiner, A. W., S. Gandolfi, F. J. Fattoyev, and W. G. Newton. "Using Neutron Star Observations to Determine Crust Thicknesses, Moments of Inertia, and Tidal Deformabilities." Physical Review C, January 2015, vol. 91, no. 1.

Widanagamaachchi, W., K. Hammond, L. Lo, B. Wirth, F. Samsel, C. Sewell, J. Ahrens, and V. Pascucci.

"Visualization and Analysis of Large-Scale Atomistic Simulations of Plasma-Surface Interactions." Eurographics, January 2015.

Zhang, W., O. Tardieu, D. Grove, B. Herta, T. Kamada, V. Saraswat, and M. Takeuchi. "GLB: Lifeline-Based Global Load Balancing Library in x10." PPAA "14 Proceedings of the First Workshop on Parallel Programming for Analytics Applications, January 2015, 31-40. 


\section{ALCF PROJECTS}

\section{INCITE PROJECTS}

\section{Biological Sciences \\ Multiscale Simulations \\ of Human Pathologies \\ George Karniadakis \\ Brown University \\ 70 Million Core-Hours \\ (ALCF: 45M; OLCF: 25M)}

Studies of Large Conformational Changes in Biomolecular Machines Benoît Roux

The University of Chicago

120 Million Core-Hours

\section{Chemistry}

\section{Catalyst Support Interactions}

Frank Abild-Pedersen

Stanford University

50 Million Core-Hours

First-Principles Simulations of High-Speed Combustion and Detonation

Alexei Khokhlov

The University of Chicago

150 Million Core-Hours

Towards Breakthroughs in Protein Structure Calculation and Design David Baker

University of Washington

80 Million Core-Hours

\section{Computer Science}

Dynamic and Adaptive Parallel Programming for Exascale Research Robert Harrison Brookhaven National Laboratory 15 Million Core-Hours
Performance Evaluation and Analysis Consortium End Station Leonid Oliker Lawrence Berkeley

National Laboratory

90 Million Core-Hours

(ALCF: 45M; OLCF: 45M)

Scalable System Software for Parallel Programming

Robert Latham

Argonne National Laboratory

25 Million Core-Hours

\section{Earth Science}

Accelerated Climate Modeling for Energy

Mark Taylor

Sandia National Laboratories

190 Million Core-Hours

(ALCF: 140M; OLCF: 50M)

\section{CESM Century-Scale}

Climate Experiments with

a High-Resolution Atmosphere

Warren Washington

National Center for

Atmospheric Research

200 Million Core-Hours

(ALCF: 200M; OLCF: 0)

Frontiers in Planetary and Stellar Magnetism through High-Performance Computing Jonathan Aurnou

University of California, Los Angeles

83 Million Core-Hours

High Frequency Ground

Motion Simulation for

Seismic Hazard Analysis

Thomas Jordan

University of Southern California

167 Million Core-Hours

(ALCF: 48M; OLCF: 119M)

\section{Engineering}

Adaptive Detached Eddy Simulation of a High Lift Wing with Active Flow Control

Kenneth Jansen

University of Colorado Boulder

70 Million Core-Hours

Direct Numerical Simulations and Robust Predictions of Cloud Cavitation Collapse

Petros Koumoutsakos

Swiss Federal Institute of Technology 88 Million Core-Hours

DNS/LES of Complex

Turbulent Flows

Krishnan Mahesh

University of Minnesota

100 Million Core-Hours

Large-Eddy Simulations of Combustor Liner Flows

Anne Dord

GE Global Research

89 Million Core-Hours

Large-Eddy Simulation of the Bachalo-Johnson Flow, with Shock-Induced Separation Philippe Spalart

Boeing

135 Million Core-Hours

Parameter Studies of Boussinesq Flows

Susan Kurien

Los Alamos National Laboratory 44 Million Core-Hours

\section{Materials Science}

Computational Spectroscopy of Heterogeneous Interfaces Giulia Galli

The University of Chicago

180 Million Core-Hours 
Non-Covalent Bonding in Complex Molecular Systems with Quantum Monte Carlo

Dario Alfè

University College London

148 Million Core-Hours

(ALCF: 68M; OLCF: 80M)

Petascale Simulations of Self-Healing Nanomaterials

Rajiv Kalia

University of Southern California

180 Million Core-Hours

Predictive Materials Modeling for Li-Air Battery Systems

Larry Curtiss

Argonne National Laboratory

50 Million Core-Hours

QMC Simulations DataBase for Predictive Theory and Modeling

David Ceperley

University of Illinois

at Urbana-Champaign

185 Million Core-Hours

(ALCF: 100; OLCF: 85)

Reactive MD Simulations of Electrochemical Oxide Interfaces at Mesoscale

Subramanian Sankaranarayanan

Argonne National Laboratory

40 Million Core-Hours

Simulation of Correlated Electrons for Superconducting Materials

Lucas Wagner

University of Illinois

at Urbana-Champaign

106 Million Core-Hours

SiO2 Fracture: Chemomechanics with a Machine Learning Hybrid QM/MM Scheme

James Kermode

King's College London

125 Million Core-Hours

State-of-the-Art Simulations

of Liquid Phenomena

Mark Gordon

lowa State University

200 Million Core-Hours

\section{Physics}

Accelerator Modeling for Discovery

James Amundson

Fermilab

60 Million Core-Hours

Cosmic Reionization on Computers

Nickolay Gnedin

Fermilab

74 Million Core-Hours

Cosmological Simulations

for Large-Scale Sky Surveys

Salman Habib

Argonne National Laboratory

160 Million Core-Hours

(ALCF: 80M; OLCF: 80M)

High-Fidelity Simulation

of Tokamak Edge Plasma Transport

Choong-Seock Chang

Princeton Plasma Physics Laboratory

270 Million Core-Hours

(ALCF: 100M; OLCF: 170M)

Lattice QCD

Paul Mackenzie

Fermilab

280 Million Core-Hours

(ALCF: 180M; OLCF: 100M)

Nuclear Structure and Nuclear Reactions

James Vary

lowa State University

204 Million Core-Hours

(ALCF: 100M; OLCF: 104M)

Particle Acceleration in Shocks:

From Astrophysics to Laboratory In Silico

Frederico Fiuza

SLAC National Accelerator Laboratory

110 Million Core-Hours

Petascale Simulation

of Magnetorotational

Core-Collapse Supernovae

Sean Couch

Michigan State University

50 Million Core-Hours
Petascale Simulations of Laser

Plasma Interaction Relevant to IFE

Frank Tsung

University of California, Los Angeles

90 Million Core-Hours

Quark Flavors and Conserved

Charges at Finite Density

in the QCD Phase Diagram

Rene Bellwied

University of Houston

150 Million Core-Hours

\section{ALCC PROJECTS}

\section{Biological Sciences}

Applying Breakthroughs in Protein Structure Calculation to the Creation of Designer Enzymes

David Baker

University of Washington

200 Million Core-Hours

\section{Chemistry}

Influence of Morphology

on Proton Transport

in Proton Exchange Membrane

Gregory Voth

The University of Chicago/

Argonne National Laboratory

57.6 Million Core-Hours

Large-Scale Turbulent

Clean Coal Combustion

Martin Berzins

University of Utah

40 Million Core-Hours

(ALCF: 10M; OLCF: 30M)

\section{Computer Science}

Hobbes: Operating System and Runtime Research for Extreme Scale Ron Brightwell

Sandia National Laboratories

40 Million Core-Hours

(ALCF: 5M; NERSC: 5M; OLCF: 30M) 


\section{Earth Science}

Delivering the Department of Energy's Next-Generation HighResolution Earth System Model Peter Thornton

Oak Ridge National Laboratory

137 Million Core-Hours

(ALCF: 107M; OLCF: 30M)

\section{Engineering}

Amplitude Modulation of Wind Turbine Noise

Sanjiva Lele

Stanford University

36.5 Million Core-Hours

Petascale Simulations in Support of CESAR

Elia Merzari

Argonne National Laboratory

80 Million Core-Hours

Predictive Large-Eddy Simulation of Jet Fuel Atomization, High-Lift Airframes, and Reacting Supersonic Turbulent Flows on Unstructured Grids

Parviz Moin

Stanford University

120 Million Core-Hours

Understanding Secondary Motions and Their Impact in Modeling Turbulent Flows

Hassan Nagib

Illinois Institute of Technology

11 Million Core-Hours

\section{Materials Science}

Interfaces in Organic and

Hybrid Photovoltaics

Noa Marom

Tulane University

105 Million Core-Hours

(ALCF: 75M; NERSC: 30M)

Large-Scale Quantum Simulations of Electrode-Electrolyte Interfaces Giulia Galli

The University of Chicago

35 Million Core-Hours
Nanostructure-Enhanced Chemical

Reactivity and Detonation in

Energetic Materials

Aidan Thompson

Sandia National Laboratories

80 Million Core-Hours

Prediction and Design of Energy

Materials by Petascale Evolutionary

Algorithm Simulations

Giancarlo Trimarchi

Northwestern University

30 Million Core-Hours

Revealing the Reversible

Electrodeposition Mechanism

in Multivalent-Ion Batteries

Gerbrand Ceder

Massachusetts Institute of Technology

98 Million Core-Hours

\section{Physics}

Composite Higgs Theory Beyond the Standard Model and the $14 \mathrm{TeV}$

Upgrade of the Large Hadron Collider Julius Kuti

University of California, San Diego

88.7 Million Core-Hours

Cosmic Frontier Computational

End-Station

Salman Habib

Argonne National Laboratory

176 Million Core-Hours

(ALCF: 100M; NERSC: 76M)

Ion Solvation, Catalytic

Interfaces, and Extreme Aqueous

Environments: An Ab Initio Study

of Liquid Water

Robert A. DiStasio Jr.

Cornell University

350 Million Core-Hours

Simulation of Large Hadron Collider Events Using Leadership Computing Thomas LeCompte

Argonne National Laboratory

52 Million Core-Hours

(ALCF: 50M; NERSC: 2M)
Turbulent Multiphase Flows for Nuclear Reactor Safety Igor A. Bolotnov

North Carolina State University

76.8 Million Core-Hours

Understanding Helium Plasma

Mediated Tungsten Surface

Response to Better Predict

Fusion Plasma Facing Component

Performance in ITER

Brian Wirth

University of Tennessee

96 Million Core-Hours

(ALCF: 66M; OLCF: 30M)

Validation Studies of Gyrokinetic Simulations to Understand the

Coupling of Ion and Electron Scale Turbulence in Tokamak Plasmas

Christopher Holland

University of California, San Diego

140 Million Core-Hours

(ALCF: 90M; NERSC: 50M)

\section{ALCC PROJECTS}

\section{Chemistry}

Anomalous Density Properties and Ion Solvation in Liquid Water: A Path-Integral A $\boldsymbol{b}$ Initio Study

Robert A. DiStasio Jr.

Cornell University

175 Million Core-Hours

Computational Design of Interfaces for Photovoltaics

Noa Marom

Tulane University

120 Million Core-Hours

(ALCF: 100M; NERSC: 20M)

PT-Symmetric Quantum Mechanics for Real-Time Electron Transport Simulations

Hanning Chen

George Washington University

16 Million Core-Hours 


\section{Computer Science}

Demonstration of the Scalability of Programming Environments By Simulating Multi-Scale Applications Robert Voigt

Leidos Inc.

167 Million Core-Hours

(ALCF: 127M; OLCF: 40M)

Performance Analysis, Modeling and Scaling of HPC Applications and Tools

Abhinav Bhatele

Lawrence Livermore

National Laboratory

29.4 Million Core-Hours

(ALCF: 20.1M; OLCF: 9.3M)

Portable Application Development for Next-Generation Supercomputer Architectures

Tjerk Straatsma

Oak Ridge National Laboratory

160 Million Core-Hours

(ALCF: 60M; NERSC: 40M;

OLCF: 60M)

\section{Earth Science}

Delivering the Department of Energy's Next-Generation HighResolution Earth System Model Peter Thornton

Oak Ridge National Laboratory

165 Million Core-Hours

(ALCF: 110M; OLCF: 55M)

Validation of RAP/HRRR for the Wind Forecast Improvement Project II Joe Olson

National Oceanic and

Atmospheric Administration

15 Million Core-Hours

\section{Engineering}

Advancing Internal Combustion Engine Simulations Using Sensitivity Analysis Sibendu Som Argonne National Laboratory 60 Million Core-Hours
Computational Design of Novel Multiscale Concrete Rheometers William George

National Institute of Standards

and Technology

50 Million Core-Hours

Credible Predictive Simulation Capabilities for Advanced Clean Energy Technology Development through Uncertainty Quantification Aytekin Gel

ALPEMI

111.5 Million Core-Hours

High-Fidelity Computations of Fuel Assemblies Subjected to Seismic Loads

Elias Balaras

George Washington University

34 Million Core-Hours

Large-Eddy Simulation of Turbine Internal Cooling Passages

Gustavo Ledezma

GE Global Research

6 Million Core-Hours

Toward a Longer-Life Core: Thermal-Hydraulic CFD Simulations of Deformed Fuel Assemblies

Elia Merzari

Argonne National Laboratory

72 Million Core-Hours

\section{Materials Science}

First-Principles Large-Scale Simulations of Interfaces for Energy Conversion and Storage

Marco Govoni

The University of Chicago/

Argonne National Laboratory

75 Million Core-Hours

Large-Scale Ab Initio Simulation of Crystalline Defects in Mg-alloys

Kaushik Bhattacharya

Caltech

20 Million Core-Hours

Predictive Modeling of Functional Nanoporous Materials

J. Ilja Siepmann

University of Minnesota

120 Million Core-Hours
Revealing the Reversible Electrodeposition Mechanism in Multivalent-ion Batteries Gerbrand Ceder

Massachusetts Institute of Technology 70 Million Core-Hours

\section{Physics}

Cosmic Frontier

Computational End-Station

Salman Habib

Argonne National Laboratory

115 Million Core-Hours

(ALCF: 65M; NERSC: 15M; OLCF: 35M)

An End-Station for Intensity and Energy Frontier Experiments and Calculations

Thomas LeCompte

Argonne National Laboratory

78 Million Core-Hours

(ALCF: 62; NERSC: 16M)

Hadronic Light-By-Light Scattering Contribution to the Muon

Anomalous Magnetic Moment from Lattice QCD with Chiral Fermions

Thomas Blum

University of Connecticut

175 Million Core-Hours

Large-Eddy Simulation and

Direct Numerical Simulation

of Fluid Induced Loads on Reactor Vessel Internals

Milorad Dzodzo

Westinghouse

40 Million Core-Hours

Understanding Helium-Hydrogen Plasma Mediated Tungsten Surface Response to Predict Fusion Plasma Facing Component Performance in ITER

Brian Wirth

University of Tennessee, Knoxville 116 Million Core-Hours

(ALCF: 80M; OLCF: 36M)

Validation Simulations of Macroscopic Burning-Plasma Dynamics

Jacob King

Tech-X

40 Million Core-Hours 


\section{DIRECTOR'S}

\section{DISCRETIONARY PROJECTS}

The following list provides a sampling of the many Director's Discretionary

projects at the ALCF.

\section{Biological Sciences}

Binding Affinity Calculations

of Estrogen Receptor Against

FDA-Approved Drugs

Sichun Yang

Case Western Reserve University

8 Million Core-Hours

Computing Three-Dimensional Structures of Large RNA from Small Angle X-Ray Scattering Data and Secondary Structure

Yun-Xing Wang

National Cancer Institute

15 Million Core-Hours

Correlating Experimentally Measured Molecular Dynamics with Computational Trajectories: Understanding Dynamic Allostery in Ubiqu

R. Andrew Byrd

National Cancer Institute

5 Million Core-Hours

Developing Novel Umbrella Sampling/Solute Tempering Algorithms in NAMD

Sunhwan Jo

Argonne National Laboratory

4.1 Million Core-Hours

Highly Parallel Macromolecular Conformational Searches and Energy Evaluations with the CHARMM Program

Robert J. Petrella

Harvard University

10 Million Core-Hours

Long-Time MD Simulation of Protein Structural Function

Ruth Nussinov

National Cancer Institute

2 Million Core-Hours
Virus Calculations with FMO

Yuri Alexeev

Argonne National Laboratory

15 Million Core-Hours

\section{Chemistry}

Large-Scale Combustion

Preparatory Access

Gabriel Staffelbach

CERFACS

12 Million Core-Hours

Modeling Nonadiabatic SpinForbidden Reaction Mechanisms in Metal-Sulfur Proteins

Sergey Varganov

University of Nevada, Reno

2 Million Core-Hours

Quantum Monte Carlo

Applied to Lithium Hyperoxides in Li-Air Batteries

John J. Low

Argonne National Laboratory

10 Million Core-Hours

Shift-and-Invert Parallel Spectral Transformation Eigensolver

Murat Keceli

Argonne National Laboratory

500,000 Core-Hours

Solving Petascale Public Health \& Safety Problems Using Uintah Martin Berzins

University of Utah

1 Million Core-Hours

\section{Computer Science}

ExaHDF5: Advancing HDF5 HPC I/O to Enable Scientific Discovery

Venkatram Vishwanath

Argonne National Laboratory

5 Million-Core Hours

Performance Studies of

Three-Dimensional Fast Fourier

Transforms Using Overlap of Communication with Computation Dmitry Pekurovsky University of California, San Diego 2.5 Million Core-Hours
SciDAC Scalable Data Management Analysis and Visualization

Michael E. Papka

Argonne National Laboratory

3.25 Million Core-Hours

\section{Earth Science}

Scalability Study for NUMA

(Non-Hydrostatic Unified Model

of the Atmosphere)

Andreas Mueller

Naval Postgraduate School

5 Million Core-Hours

\section{Energy Technologies}

GTRI- and NEAMS-Related

Production Tests and Runs

Micheal A. Smith

Argonne National Laboratory

10 Million Core-Hours

\section{Engineering}

Aircraft Fuel Burn Reduction Using Minute Roughness Elements Ali Uzun

Florida State University

1 Million Core-Hours

DNS of Compressible Turbulent Boundary Layers

Jonathan Poggie

Air Force Research Laboratory

5 Million Core-Hours

DNS of Multi-Mode

Rayleigh-Taylor Instability

Maxwell Hutchinson

The University of Chicago

5 Million Core-Hours

Extreme-Scale Unstructured Mesh CFD Workflow

Cameron Smith

Rensselaer Polytechnic Institute

5 Million Core-Hours

Large-Eddy Simulation of Crackling Supersonic Jets

Joseph Nichols

University of Minnesota

10 Million Core-Hours 
Numerical Simulation of Acoustic Radiation from High-Speed Turbulent Boundary Layers

Lian Duan

Missouri University

of Science and Technology

2 Million Core-Hours

Performance Improvement of CFD Code CONVERGE on BG/Q Systems

Marta García and Sibendu Som

Argonne National Laboratory

6 Million Core-Hours

\section{Primary Atomization DNS}

of ECN's Spray A

Marcus Herrmann

Arizona State University

5 Million Core-Hours

Turbulent Rayleigh-Benard

Convection at High Rayleigh

and Low Prandtl Numbers

Janet Scheel

Occidental College

1 Million Core-Hours

\section{Materials Science}

Collective $\mathrm{I} / \mathrm{O}$ and Bond Analysis

Code Development on SiC

Nanoparticle Oxidation

Ying Li

Argonne National Laboratory

5 Million Core-Hours

Electronic Response to Particle

Radiation in Semiconductor Systems

Andre Schleife

University of Illinois

at Urbana-Champaign

16 Million Core-Hours
High-Performance Li-Air Battery

Ying Li

Argonne National Laboratory

5 Million Core-Hours

Integrating Simulation and Observation: Discovery Engines for Big Data

Justin Wozniak

Argonne National Laboratory

6.9 Million Core-Hours

Many-Body Stochastic Analysis of Semiconductor Bulk and

Defect Properties

Elif Ertekin

University of Illinois

at Urbana-Champaign

2 Million Core-Hours

\section{Mathematics}

Parallel Multiscale Simulations of Advanced Steel Materials

Axel Klawonn

University of Cologne; Oliver

Rheinbach, Technische Universität

Bergakademie Freiberg

3 Million Core-Hours

Scalable Domain Decomposition

Methods for Computational

Cardiology and

Isogeometric Analysis

Luca F. Pavarino

University of Milan

1.3 Million Core-Hours

\section{Physics}

Calculation of Nuclear Matrix

Element of Neutrinoless

Double-Beta Decay

Jun Terasaki

University of Tsukuba

3.8 Million Core-Hours

DNS Simulations of Turbulent

Rayleigh-Taylor Unstable Flames

Using Nek5000

Elizabeth P. Hicks

Epsilon Delta Labs

2.5 Million Core-Hours

Effective Interactions

in Coulombic Systems with

Highly Disparate Particle Sizes

Monica Olvera de la Cruz

Northwestern University

3.1 Million Core-Hours

Extreme-Scale

Turbulence Simulations

William M. Tang

Princeton Plasma Physics Laboratory

60 Million Core-Hours

Mira Simulations of High-Intensity

Laser Experiments to Study Turbulent Amplification of Magnetic Fields

Don Q. Lamb

The University of Chicago

10 Million Core-Hours

Moving Mesh Simulations

of Gravito-Turbulence

in Global Proto-Planetary Disks

Andrew MacFadyen

New York University

250,000 Core-Hours 


\section{About Argonne Leadership Computing Facility}

The Leadership Computing Facility Division operates the Argonne Leadership Computing Facility - the ALCF-as part of the U.S. Department of Energy's (DOE) effort to provide leadership-class computing resources to the scientific community. The ALCF is supported by DOE's Office of Science, Advanced Scientific Computing Research (ASCR) program.

\section{About Argonne National Laboratory}

Argonne is a U.S. Department of Energy laboratory managed by UChicago Argonne, LLC under contract DE-AC02-06CH11357. The Laboratory's main facility is outside Chicago, at 9700 South Cass Avenue, Argonne, Illinois 60439. For information about Argonne and its pioneering science and technology programs, see www.anl.gov.

\section{Availability of this Report (ANL/ALCF-16/1)}

Online Access: U.S. Department of Energy (DOE) reports produced after 1991 and a growing number of pre-1991 documents are available free via DOE's SciTech Connect (http://www.osti.gov/scitech/).

Reports not in digital format may be purchased by the public from

the National Technical Information Service (NTIS):

U.S. Department of Commerce National Technical Information Service 5301 Shawnee Rd

Alexandra, VA 22312

www.ntis.gov phone | 800.553.NTIS (6847) or 703.605 .6000 fax | 703.605.6900

orders@ntis.gov

Reports not in digital format are available to DOE and DOE contractors from

\author{
the Office of Scientific and Technical \\ Information (OSTI):
}

U.S. Department of Energy

Office of Scientific and Technical Information P.O. Box 62

Oak Ridge, TN 37831-0062

phone | 865.576.8401

fax | 865.576.5728

reports@adonis.osti.gov

\section{Disclaimer}

This report was prepared as an account of work sponsored by an agency of the United States Government. Neither the United States Government nor any agency thereof, nor UChicago Argonne, LLC, nor any of their employees or officers, makes any warranty, express or implied, or assumes any legal liability or responsibility for the accuracy, completeness, or usefulness of any information, apparatus, product, or process disclosed, or represents that its use would not infringe privately owned rights. Reference herein to any specific commercial product, process, or service by trade name, trademark, manufacturer, or otherwise, does not necessarily constitute or imply its endorsement, recommendation, or favoring by the United States Government or any agency thereof. The views and opinions of document authors expressed herein do not necessarily state or reflect those of the United States Government or any agency thereof, Argonne National Laboratory, or UChicago Argonne, LLC. 



\section{Argonne}

\section{CONTACT}

media@alcf.anl.gov

alcf.anl.gov 